\title{
A Bottom Up Approach for the Rational Loading of Linear Oligomers and Polymers with Lanthanides
}

Mohsen Mirzakhani, ${ }^{a}$ Homayoun Nozary, ${ }^{a}$ Soroush Naseri, ${ }^{a}$ Céline Besnard, ${ }^{b}$ Laure Guénée ${ }^{b}$ and Claude Piguet ${ }^{a *}$

${ }^{a}$ Department of Inorganic and Analytical Chemistry. University of Geneva, 30 quai E. Ansermet CH1211 Geneva 4 (Switzerland).

${ }^{b}$ Laboratory of Crystallography. University of Geneva, 24 quai E. Ansermet. CH-1211 Geneva 4 (Switzerland).

*Email: claude.piguet@unige.ch

\section{Supporting Information}

(69 pages)

\section{$\underline{\text { Table of contents }}$}

Experimental section

S2-S16

Ligand characterization (NMR and X-ray)

S17-S22

Complexes characterization (NMR and X-ray)

S23-S58

Thermodynamic NMR titrations

S59-S67

Matrix transfer predictions

S68-S69 


\section{Experimental Section}

\section{Solvents and starting materials}

All commercial chemicals were purchased from Strem, Acros, Fluka AG, and Sigma-Aldrich, and used without additional purification. The references ligands $\mathbf{M} 4,{ }^{\mathrm{S} 1} \mathbf{L 1}^{\mathrm{S} 2}$ and $\mathbf{L 2}{ }^{\mathrm{S} 2}$ were prepared following literature procedures. The hexafluoroacetylacetonate salts $\left[\operatorname{Ln}(\mathrm{hfa})_{3} \mathrm{dig}\right]$ were synthetized from the corresponding oxides (Aldrich, 99.99\%). ${ }^{\mathrm{S} 3}$ Dichloromethane and $N, N$-dimethylformamide were dried over calcium hydride. Silica gel plates Merck $60 \mathrm{~F}_{254}$ were used for thin layer chromatography and Fluka silica gel $60(0.04-0.063 \mathrm{~mm})$ and Acros neutral activated alumina (0.050$0.200 \mathrm{~mm}$ ) was used for preparative column chromatography.

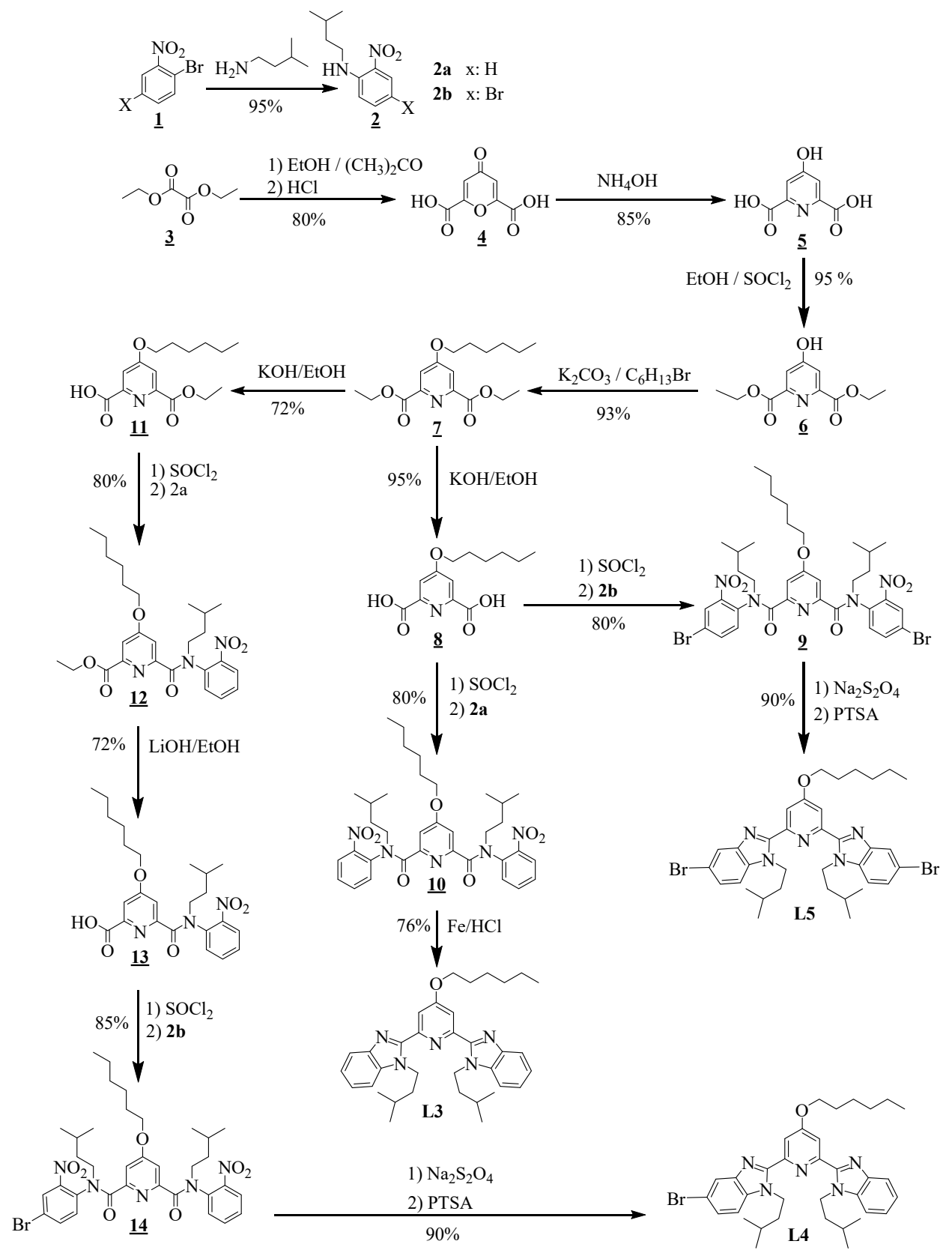

Scheme S1 Synthesis of ligands L3-L5. 


\section{Preparation of $\mathrm{N}$-isopentyl-2-nitroaniline (2a)}

1-bromo-2-nitrobenzene (11.31 g, $56 \mathrm{mmol})$ and 3-methylbutan-1-amine (23.29 g, $267 \mathrm{mmol}$ ) were heated in an autoclave at $110^{\circ} \mathrm{C}$ for $24 \mathrm{~h}$. The dark red mixture was evaporated to dryness, then extracted with dichloromethane $(100 \mathrm{~mL})$ and washed with half-saturated aq. $\mathrm{NH}_{4} \mathrm{Cl}$ solution $(3 \times 50 \mathrm{~mL})$ and water $(50 \mathrm{~mL})$. The organic layer was dried over anhydrous $\mathrm{Na}_{2} \mathrm{SO}_{4}$, filtered, and evaporated to give $\mathrm{N}$ -

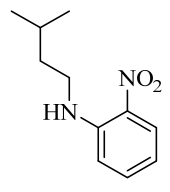
$\underline{2 \mathrm{a}}$ isopentyl-2-nitroaniline (2a, $11.1 \mathrm{~g}, 53.29 \mathrm{mmol}$, yield 95\%) as a red oil.

${ }^{1} \mathrm{H}$ NMR (400 MHz, chloroform- $d$ ), $\delta / \mathrm{ppm}: 8.15\left(\mathrm{dd},{ }^{3} J=8.6,{ }^{4} \mathrm{~J}=1.5 \mathrm{~Hz}, 1 \mathrm{H}\right), 8(\mathrm{~s}, 1 \mathrm{H}), 7.41\left(\mathrm{t},{ }^{3} \mathrm{~J}\right.$ $=8.6 \mathrm{~Hz}, 1 \mathrm{H}), 6.83\left(\mathrm{~d},{ }^{3} J=8.6 \mathrm{~Hz}, 1 \mathrm{H}\right), 6.61\left(\mathrm{t},{ }^{3} J=7.0 \mathrm{~Hz}, 1 \mathrm{H}\right), 3.29\left(\mathrm{q},{ }^{3} J=7.3 \mathrm{~Hz}, 2 \mathrm{H}\right), 1.75(\mathrm{n}$, $\left.{ }^{3} J=6.6 \mathrm{~Hz}, 1 \mathrm{H}\right), 1.61\left(\mathrm{q},{ }^{3} J=7.1 \mathrm{~Hz}, 2 \mathrm{H}\right), 0.96\left(\mathrm{~d},{ }^{3} J=6.6 \mathrm{~Hz}, 6 \mathrm{H}\right)$. ESI-MS (positive mode $/ \mathrm{CH}_{2} \mathrm{Cl}_{2}$ ), $m / z: 209\left([\mathrm{M}+\mathrm{H}]^{+}\right) .{ }^{\mathrm{S} 2}$

\section{Preparation of 4-bromo-N-isopentyl-2-nitroaniline (2b)}

1,4-dibromo-2-nitrobenzene (15.98 g, $56 \mathrm{mmol}$ ) and 3-methylbutan-1-amine (23.29 $\mathrm{g}, 267 \mathrm{mmol}$ ) were heated in an autoclave at $110^{\circ} \mathrm{C}$ for $24 \mathrm{~h}$. The dark red mixture was evaporated to dryness, then extracted with dichloromethane $(100 \mathrm{~mL})$ and washed with half saturated aq. $\mathrm{NH}_{4} \mathrm{Cl}$ solution $(3 \times 50 \mathrm{~mL})$ and water $(50 \mathrm{~mL})$. The

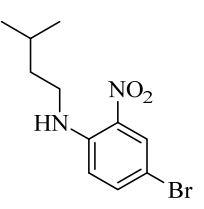

$\underline{2 b}$ organic layer was dried over anhydrous $\mathrm{Na}_{2} \mathrm{SO}_{4}$, filtered, and evaporated. The resulting red oil was crystallized in hexane to give 4-bromo- $N$-isopentyl-2-nitroaniline (2b, $15.2 \mathrm{~g}$, $52.9 \mathrm{mmol}$, yield 95\%) as red crystals.

${ }^{1} \mathrm{H}$ NMR (400 MHz, chloroform- $d$ ), $\delta / \mathrm{ppm}: 8.29\left(\mathrm{~d},{ }^{4} J=2.3 \mathrm{~Hz}, 1 \mathrm{H}\right), 7.98(\mathrm{~s}, 1 \mathrm{H}), 7.47\left(\mathrm{dd},{ }^{3} J=9.2\right.$, $\left.{ }^{4} J=2.3 \mathrm{~Hz}, 1 \mathrm{H}\right), 6.74\left(\mathrm{~d},{ }^{3} J=9.2 \mathrm{~Hz}, 1 \mathrm{H}\right), 3.27\left(\mathrm{q},{ }^{3} J=7.3 \mathrm{~Hz}, 2 \mathrm{H}\right), 1.73\left(\mathrm{n},{ }^{3} J=6.8 \mathrm{~Hz}, 1 \mathrm{H}\right), 1.61$ $\left(\mathrm{q},{ }^{3} J=7.1 \mathrm{~Hz}, 2 \mathrm{H}\right), 0.96\left(\mathrm{~d},{ }^{3} J=6.6 \mathrm{~Hz}, 6 \mathrm{H}\right)$. ESI-MS (positive mode $\left./ \mathrm{CH}_{2} \mathrm{Cl}_{2}\right), m / z: 288\left([\mathrm{M}+\mathrm{H}]^{+}\right) .{ }^{\mathrm{S} 2}$

\section{Preparation of chelidonic acid (4)}

Sodium $(23.5 \mathrm{~g}, 1.02 \mathrm{~mol})$ was dissolved in $360 \mathrm{~mL}$ of dry ethanol. A mixture of $29.0 \mathrm{~g}(38 \mathrm{~mL}, 0.5 \mathrm{~mol})$ of dry acetone and $155.0 \mathrm{~g}(144 \mathrm{~mL}, 1.06 \mathrm{~mol})$ of diethyloxalate were dropwise added in 15 min. During the addition, a yellow precipitate was formed. The reaction mixture was kept at $60{ }^{\circ} \mathrm{C}$ for one hour to

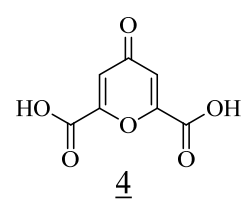
complete the reaction. Then, $200 \mathrm{ml}$ of $37 \%$ aqueous $\mathrm{HCl}$ and $100 \mathrm{~mL}$ of water were added, and the solution was stirred at $50{ }^{\circ} \mathrm{C}$ for one day. About $450 \mathrm{~mL}$ of aqueous ethanol was removed under reduced pressure. $300 \mathrm{~mL}$ of water and $50 \mathrm{~mL}$ of $37 \%$ aqueous $\mathrm{HCl}$ were then added to this mixture. The mixing was maintained about three days until a silica gel TLC (eluent: 10\% aqueous $\mathrm{NaCl} /$ ethanol: $3 / 7(\mathrm{v} / \mathrm{v}))$ showed only one spot $(\mathrm{Rf}=0.65)$. After cooling down, the insoluble crystals were filtered off, washed first with water then with cold acetone. The crude product was recrystallized from boiling water $(2.5 \mathrm{~L})$, using charcoal $(10 \mathrm{~g})$ to give $73.6 \mathrm{~g}(0.4 \mathrm{~mol}$, yield $80 \%)$ of chelidonic acid (4) as a white microcrystalline powder. 
${ }^{1} \mathrm{H}$ NMR (400 MHz, deuterium oxide) $\delta 7.03$ (s, 2H); ESI-MS (DMSO), m/z: $185.1\left(\left[\mathrm{M}^{+} \mathrm{H}\right]^{+}\right), 207.1$ $\left([\mathrm{M}+\mathrm{Na}]^{+}\right) .{ }^{\mathrm{S} 4}$

\section{Preparation of chelidamic acid (5)}

$300 \mathrm{~mL}$ aqueous solution of $\mathrm{NH}_{3}(25 \%)$ was dropwise added onto chelidonic acid $(4,30 \mathrm{~g}, 0.162 \mathrm{~mol})$ at $0{ }^{\circ} \mathrm{C}$. The resulting white suspension was mixed at room temperature for 48 hours (after five hours, the suspension became orange). The excess of aqueous ammonia solution was removed under reduced pressure

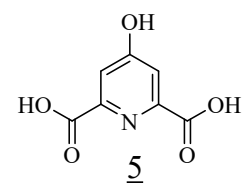
and the residue was boiled with $300 \mathrm{~mL}$ of water containing charcoal $(7 \mathrm{~g})$ and filtered. The cold solution was acidified with $37 \%$ aqueous $\mathrm{HCl}$ to about $\mathrm{pH}=1$. The white crystals were filtered off, washed three times with ice-cold water and dried under vacuum at $150{ }^{\circ} \mathrm{C}$ for 24 hours to give 23.1 $\mathrm{g}(12.6 \mathrm{mmol}, 85 \%)$ of chelidamic acid (5). Rf $=0.35$ (silica gel TLC, $3 / 7$ (v/v) 10\% aqueous $\mathrm{NaCl} /$ ethanol).

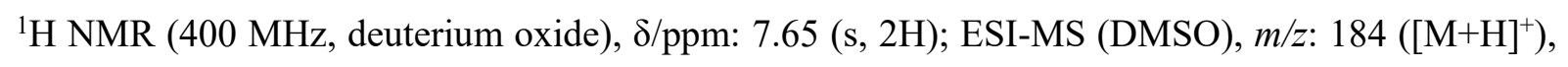
$206.1\left([\mathrm{M}+\mathrm{Na}]^{+}\right){ }^{\mathrm{S} 4}$

\section{Preparation of diethyl 4-hydroxypyridine-2, 6-dicarboxylate (6)}

Thionyl chloride $(40.2 \mathrm{~mL}, 554.5 \mathrm{mmol})$ were dropwise added to a roundbottomed flask containing chelidamic acid $(5,16 \mathrm{~g}, 87 \mathrm{mmol})$ and absolute ethanol $(160 \mathrm{~mL})$ at $0{ }^{\circ} \mathrm{C}$. The resulting mixture was warmed to reach room temperature, stirred at this temperature for $18 \mathrm{~h}$, and then heated at reflux

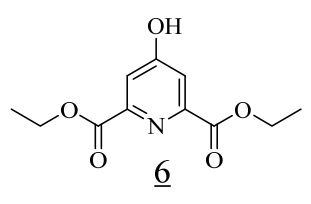
$\left(80^{\circ} \mathrm{C}\right)$ for $2 \mathrm{~h}$. The solvent was evaporated under reduced pressure and distilled water was added to the crude product at $0{ }^{\circ} \mathrm{C}$. The mixture was neutralized with a saturated aqueous solution of $\mathrm{Na}_{2} \mathrm{CO}_{3}$. The precipitate was filtered, washed with $50 \%$ aqueous ethanol $(20 \mathrm{~mL})$ and water $(20 \mathrm{~mL})$. The final product was dried under reduced pressure to give diethyl 4-hydroxypyridine-2, 6-dicarboxylate (6, $19.7 \mathrm{~g}, 82.3 \mathrm{mmol}$, yield 95\%).

${ }^{1} \mathrm{H}$ NMR (400 MHz, methylene chloride- $d_{2}$ ), $\delta / \mathrm{ppm}: 7.48$ (s, 1H), 4.37 (q, $\left.{ }^{3} J=7.1 \mathrm{~Hz}, 4 \mathrm{H}\right), 1.34$ (t, $\left.{ }^{3} J=7.1 \mathrm{~Hz}, 6 \mathrm{H}\right)$; ESI-MS (positive mode $\left./ \mathrm{CH}_{2} \mathrm{Cl}_{2}\right), m / z: 240.35\left([\mathrm{M}+\mathrm{H}]^{+}\right) .{ }^{\mathrm{S} 5}$

\section{Preparation of diethyl 4-(hexyloxy) pyridine-2,6-dicarboxylate(7)}

1-bromohexane (11.4 g, $68.8 \mathrm{mmol})$ was added to a round-bottomed flask containing diethyl 4-hydroxypyridine-2,6-dicarboxylate 6 (15 g, 62.7 mmol) and $\mathrm{K}_{2} \mathrm{CO}_{3}(34.6 \mathrm{~g}, 251 \mathrm{mmol})$ in acetonitrile $(500 \mathrm{~mL})$. The

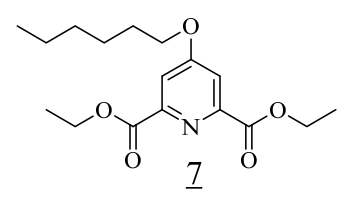
resulting mixture was heated to reflux for $16 \mathrm{~h}$. After cooling to room temperature, the solvent was evaporated under reduced pressure, $100 \mathrm{~mL}$ water was added and the resulting solution was extracted with dichloromethane $(3 \times 100 \mathrm{~mL})$. The combined organic phases were dried over $\mathrm{MgSO}_{4}$, filtered, and evaporated to give a yellowish oil of diethyl 4-(hexyloxy) pyridine-2,6-dicarboxylate (7, $18.8 \mathrm{~g}, 58.1 \mathrm{mmol}$, yield 93\%). 
${ }^{1} \mathrm{H}$ NMR (400 MHz, dichloromethane-d 2 ), $\delta / \mathrm{ppm}: 7.74$ (s, 2H), 4.44 (q, $\left.{ }^{3} J=7.1 \mathrm{~Hz}, 4 \mathrm{H}\right), 4.13\left(\mathrm{t},{ }^{3} J=\right.$ $6.5 \mathrm{~Hz}, 2 \mathrm{H}), 1.87-1.77(\mathrm{~m}, 2 \mathrm{H}), 1.39-1.51(\mathrm{~m}, 8 \mathrm{H}), 1.31-1.38(\mathrm{~m}, 4 \mathrm{H}), 0.9\left(\mathrm{t},{ }^{3} J=7.1 \mathrm{~Hz}, 3 \mathrm{H}\right)$; ESI-MS (positive mode/ $\left.\mathrm{CH}_{2} \mathrm{Cl}_{2}\right): m / z: 362\left([\mathrm{M}+\mathrm{K}]^{+}\right) .{ }^{\mathrm{S}} 6$

\section{Preparation of 4-(hexyloxy)pyridine-2,6-dicarboxylic acid (8)}

4-(hexyloxy)pyridine-2,6-dicarboxylate 7 (5.7 g, $17.6 \mathrm{mmol})$ was dissolved in ethanol $(200 \mathrm{~mL})$ in an ice bath. A solution of potassium hydroxide $(2.4 \mathrm{~g}, 44$ $\mathrm{mmol})$ in ethanol:water $(90: 10 \mathrm{~mL})$ was added to the cooled solution and the resulting suspension was stirred at room temperature for $2 \mathrm{~h}$. After completion

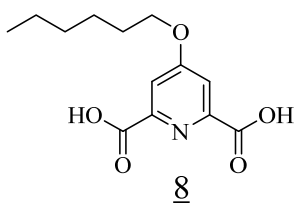
of reaction, ethanol was evaporated and water $(30 \mathrm{~mL})$ added to the white residue and $\mathrm{pH}$ adjusted to 1.0 with aq. $10 \% \mathrm{HCl}$. The resulting precipitate was filtered, washed with water $(2 \times 10 \mathrm{~mL})$ and dried to give 4-(hexyloxy)pyridine-2,6-dicarboxylic acid (8, $4.46 \mathrm{~g}, 16.68 \mathrm{mmol}$, yield 95\%).

${ }^{1} \mathrm{H}$ NMR (400 MHz, DMSO-d6), $\delta / \mathrm{ppm}: 7.69$ (s, 2H), $4.22\left(\mathrm{t},{ }^{3} J=6.5 \mathrm{~Hz}, 2 \mathrm{H}\right), 1.81-1.68$ (m, 2H), $1.50-1.37(\mathrm{~m}, 2 \mathrm{H}), 1.27-1.36(\mathrm{~m}, 4 \mathrm{H}), 0.88\left(\mathrm{t},{ }^{3} \mathrm{~J}=7 \mathrm{~Hz}, 3 \mathrm{H}\right)$. ESI-MS $\left(\mathrm{CH}_{2} \mathrm{Cl}_{2}\right), m / z: 268.3$ $\left([\mathrm{M}+\mathrm{H}]^{+}\right) .{ }^{\mathrm{S} 7}$

Preparation of N2,N6-bis(4-bromo-2-nitrophenyl)-4-(hexyloxy)-N2,N6-diisopentylpyridine2,6-dicarboxamide (9)

4-(hexyloxy)pyridine-2,6-dicarboxylic acid (8, $5 \mathrm{~g}, 18.7 \mathrm{mmol})$ and DMF $(30 \mu \mathrm{L})$ were heated to reflux in thionyl chloride $(15.5$ $\mathrm{mL}, 244 \mathrm{mmol}$ ) for $1 \mathrm{~h}$. Excess of thionyl chloride was distilled from the reaction mixture, which was then co-evaporated with dry $\mathrm{CH}_{2} \mathrm{Cl}_{2}(3 \times 20 \mathrm{~mL})$ and dried under vacuum. The obtained solid

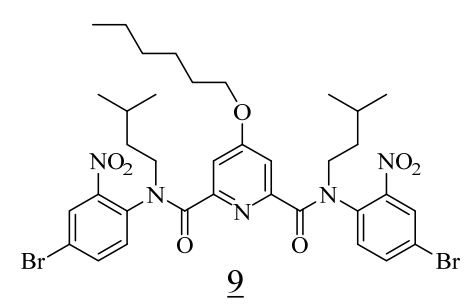
was re-dissolved in freshly distilled dry $\mathrm{CH}_{2} \mathrm{Cl}_{2}(30 \mathrm{~mL})$ and a solution of 4-bromo-N-isopentyl-2nitroaniline (2b, $11 \mathrm{~g}, 38.32 \mathrm{mmol})$ in $\mathrm{CH}_{2} \mathrm{Cl}_{2}(30 \mathrm{~mL})$ was slowly added under an inert atmosphere. The resulting mixture was heated under reflux for $24 \mathrm{~h}$ whilst the $\mathrm{pH}$ was kept close to 9 by adding small amounts of $\mathrm{N}, \mathrm{N}$-diisopropylethylamine. The cooled mixture was partitioned between $\mathrm{CH}_{2} \mathrm{Cl}_{2}$ $(50 \mathrm{~mL})$ and half-saturated aqueous $\mathrm{NH}_{4} \mathrm{Cl}(50 \mathrm{~mL})$. The organic layer was separated and the aqueous phase was further extracted with fresh $\mathrm{CH}_{2} \mathrm{Cl}_{2}(2 \times 60 \mathrm{~mL})$. The combined organic phases were dried with $\mathrm{Na}_{2} \mathrm{SO}_{4}$, filtered and evaporated to dryness. The crude product was purified by column chromatography on silica gel $\left(\mathrm{CH}_{2} \mathrm{Cl}_{2}: \mathrm{MeOH}, 100: 0 \rightarrow 99: 1\right)$ to give N2,N6-bis(4-bromo-2nitrophenyl)-4-(hexyloxy)-N2,N6-diisopentylpyridine-2,6-dicarboxamide (9, 12 g, 14.89 mmol, yield $80 \%$ ) as a yellowish oil.

${ }^{1} \mathrm{H}$ NMR (400 MHz, DMSO-d6, $393 \mathrm{~K}$ ), $\delta / \mathrm{ppm}: 8.13$ (s, 2H), 7.91 (d, $\left.{ }^{3} J=8.5 \mathrm{~Hz}, 2 \mathrm{H}\right), 7.40\left(\mathrm{~d},{ }^{3} J=\right.$ $8.3 \mathrm{~Hz}, 2 \mathrm{H}), 7.02(\mathrm{~s}, 2 \mathrm{H}), 4.09\left(\mathrm{t},{ }^{3} J=6.5 \mathrm{~Hz}, 2 \mathrm{H}\right), 3.62(\mathrm{~s}, 4 \mathrm{H}), 1.75-1.66(\mathrm{~m}, 2 \mathrm{H}), 1.63-1.21(\mathrm{~m}$, $12 \mathrm{H}), 0.98-0.62(\mathrm{~m}, 15 \mathrm{H})$. ESI-MS $\left(\mathrm{CH}_{2} \mathrm{Cl}_{2}\right), m / z: 806.4\left([\mathrm{M}+\mathrm{H}]^{+}\right) .{ }^{\mathrm{S} 8}$ 
Preparation of 2,2'-(4-(hexyloxy)pyridine-2,6-diyl)bis(5-bromo-1-isopentyl-1Hbenzo[d]imidazole) (L5)

Sodium dithionite $(10.1 \mathrm{~g}, 57.3 \mathrm{mmol})$, water $(40 \mathrm{~mL})$ and methanol $(5 \mathrm{ml})$ were mixed in a round-bottomed flask. A solution of N2,N6-bis(4-bromo-2-nitrophenyl)-4-(hexyloxy)-N2,N6diisopentylpyridine-2,6-dicarboxamide (9, $4 \mathrm{~g}, 4.9 \mathrm{mmol})$ in $\mathrm{THF}$ $(50 \mathrm{~mL})$ were added to the mixture, which was further stirred at

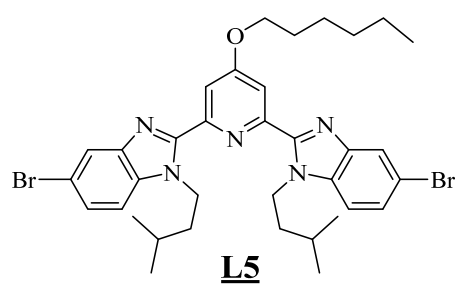
room temperature for $24 \mathrm{~h}$ under an inert atmosphere. The solvent was evaporated under reduced pressure at room temperature. Dichloromethane $(60 \mathrm{~mL})$ and aq. $10 \% \mathrm{NaHCO}_{3}(40 \mathrm{~mL})$ were added and the resulting mixture stirred for $1 \mathrm{~h}(\mathrm{pH}=6.5-7)$. The organic phase was separated and the aqueous phase further extracted with dichloromethane $(3 \times 150 \mathrm{~mL})$. The combined organic phases were dried with $\mathrm{Na}_{2} \mathrm{SO}_{4}$, filtered and evaporated under reduced pressure to dryness. The residue was dissolved in $30 \mathrm{~mL}$ xylene and p-toluene sulfonic acid $(0.7 \mathrm{~g}, 3.9 \mathrm{mmol})$ was added. The resulting mixture was heated at $120^{\circ} \mathrm{C}$ for $6 \mathrm{~h}$. The solvent was evaporated under reduced pressure to dryness and the residue partitioned between $\mathrm{CH}_{2} \mathrm{Cl}_{2}(50 \mathrm{~mL})$ and water $(30 \mathrm{~mL}), \mathrm{pH}$ was adjusted to 7.5-8.5 with $10 \%$ aq. $\mathrm{K}_{2} \mathrm{CO}_{3}$ and the organic phase was separated. The aqueous phase was further extracted with dichloromethane $(2 \times 60 \mathrm{~mL})$. The combined organic phases were dried with $\mathrm{Na}_{2} \mathrm{SO}_{4}$, filtered, and evaporated under reduced pressure to dryness. The crude product was purified by column chromatography on silica gel $\left(\mathrm{CH}_{2} \mathrm{Cl}_{2} / \mathrm{MeOH}, \quad 99.5: 0.5 \rightarrow 98.5: 1.5\right)$ to give 2,2'-(4(hexyloxy)pyridine-2,6-diyl)bis(5-bromo-1-isopentyl-1H-benzo[d]imidazole) (L5, 3.16 g, 5.01 mmol yield 90\%).

${ }^{1} \mathrm{H}$ NMR (400 MHz, chloroform-d), $\delta / \mathrm{ppm}: 7.99$ (d, $\left.{ }^{4} J=1.7 \mathrm{~Hz}, 2 \mathrm{H}\right), 7.79$ (s, 2H), 7.45 (dd, ${ }^{3} J=8.6$, $\left.{ }^{4} J=1.8 \mathrm{~Hz}, 2 \mathrm{H}\right), 7.31\left(\mathrm{~d},{ }^{3} J=8.6 \mathrm{~Hz}, 2 \mathrm{H}\right), 4.46\left(\mathrm{t},{ }^{3} J=7.9 \mathrm{~Hz}, 4 \mathrm{H}\right), 4.23\left(\mathrm{t},{ }^{3} J=6.4 \mathrm{~Hz}, 2 \mathrm{H}\right), 1.94-$ $1.77(\mathrm{~m}, 2 \mathrm{H}), 1.62-154(\mathrm{~m}, 4 \mathrm{H}), 1.53-145(\mathrm{~m}, 2 \mathrm{H}), 1.44-1.30(\mathrm{~m}, 6 \mathrm{H}), 0.92\left(\mathrm{t},{ }^{3} J=6.9 \mathrm{~Hz}, 3 \mathrm{H}\right), 0.69$ $\left(\mathrm{d},{ }^{3} \mathrm{~J}=6.6 \mathrm{~Hz}, 12 \mathrm{H}\right)$. ESI-MS $\left(\mathrm{CH}_{2} \mathrm{Cl}_{2}\right), m / z: 710.2\left([\mathrm{M}+\mathrm{H}]^{+}\right) .{ }^{\mathrm{S} 8}$

Preparation of 4-(hexyloxy)-N2,N6-diisopentyl-N2,N6-bis(2-nitrophenyl)pyridine-2,6 dicarboxamide(10)

4-(hexyloxy)pyridine-2,6-dicarboxylic acid (8, $1.7 \mathrm{~g}, 6.3 \mathrm{mmol})$ and DMF $(30 \mu \mathrm{L})$ were heated to reflux in thionyl chloride $(8.8 \mathrm{~mL}, 126$ mmol) for $1 \mathrm{~h}$. Excess thionyl chloride was distilled from the reaction mixture, which was then co-evaporated with dry $\mathrm{CH}_{2} \mathrm{Cl}_{2}(3 \times 15 \mathrm{~mL})$ and dried under vacuum. The solid was re-dissolved in freshly distilled

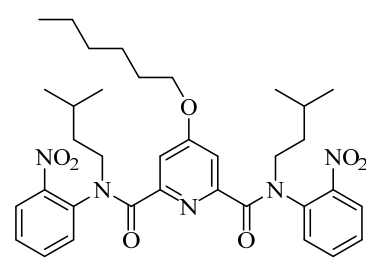

$\underline{10}$ $\mathrm{CH}_{2} \mathrm{Cl}_{2}(20 \mathrm{~mL})$ and a solution of N-isopentyl-2-nitroaniline (1a, $\left.2.9 \mathrm{~g}, 13.9 \mathrm{mmol}\right)$ in $\mathrm{CH}_{2} \mathrm{Cl}_{2}(20$ $\mathrm{mL}$ ) was dropwise added under an inert atmosphere. The resulting mixture was heated to reflux for $24 \mathrm{~h}$ and the $\mathrm{pH}$ value was kept close to 9 by adding small amounts of $N, N$-diisopropylethylamine. 
The cooled mixture was partitioned between $\mathrm{CH}_{2} \mathrm{Cl}_{2}(40 \mathrm{~mL})$ and half-saturated aqueous $\mathrm{NH}_{4} \mathrm{Cl}(40$ $\mathrm{mL}$ ), the organic layer was separated and the aqueous phase was further extracted with fresh $\mathrm{CH}_{2} \mathrm{Cl}_{2}$ $(2 \times 50 \mathrm{~mL})$. The combined organic phases were dried with $\mathrm{Na}_{2} \mathrm{SO}_{4}$, evaporated to dryness, and the crude product was purified by column chromatography on silica gel $\left(\mathrm{CH}_{2} \mathrm{Cl}_{2}: \mathrm{MeOH}, 100: 0 \rightarrow 99: 1\right)$ to give 4-(hexyloxy)-N2,N6-diisopentyl-N2,N6-bis(2-nitrophenyl)pyridine-2,6 dicarboxamide (10, $3.26 \mathrm{~g}, 5.03 \mathrm{mmol}$ yield $80 \%$ ) as a yellowish oil.

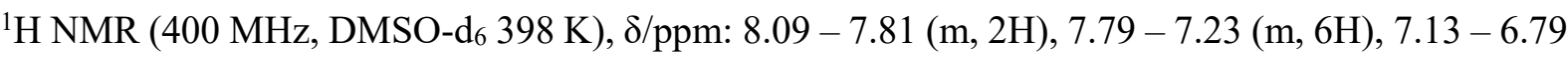
$(\mathrm{m}, 2 \mathrm{H}), 4.13-3.9(\mathrm{~m}, 2 \mathrm{H}), 3.89-3.27(\mathrm{~m}, 4 \mathrm{H}), 1.83-1.15(\mathrm{~m}, 14 \mathrm{H}), 0.97-0.60(\mathrm{~m}, 15 \mathrm{H})$. ESI$\operatorname{MS}\left(\mathrm{CH}_{2} \mathrm{Cl}_{2}\right): m / z: 648.3\left([\mathrm{M}+\mathrm{H}]^{+}\right)$.

Preparation of 2,2'-(4-(hexyloxy)pyridine-2,6-diyl)bis(1-isopentyl-1H-benzo[d]imidazole) (L3) Activated iron powder $(5.23 \mathrm{~g}, 94.23 \mathrm{mmol})$ and concentrated hydrochloric acid 37\% (19 mL, $190 \mathrm{mmol})$ were added to a solution of 4-(hexyloxy)-N2,N6-diisopentyl-N2,N6-bis(2-nitrophenyl)pyridine2,6 dicarboxamide (10, $2 \mathrm{~g}, 3.08 \mathrm{mmol})$ in ethanol/water $(600 \mathrm{~mL} / 160$ $\mathrm{mL}$ ). The mixture was refluxed for $15 \mathrm{~h}$ under an inert atmosphere,

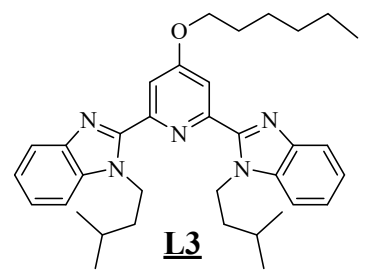
excess iron was filtered off, ethanol was distilled under vacuum, and $100 \mathrm{~mL}$ water added. A solution of $\mathrm{Na}_{2} \mathrm{H}_{2}$ EDTA $2 \mathrm{H}_{2} \mathrm{O}(16 \mathrm{~g})$ in water $(100 \mathrm{~mL})$ was poured into the resulting mixture, and dichloromethane $(300 \mathrm{~mL})$ was added. The stirred mixture was neutralized $(\mathrm{pH}=7)$ with concentrated aqueous $\mathrm{NH}_{4} \mathrm{OH}$ solution, and concentrated $\mathrm{H}_{2} \mathrm{O}_{2}$ solution $(30 \%, 2.1 \mathrm{~mL})$ was then dropwise added under vigorous stirring. The final $\mathrm{pH}$ was adjusted to 8.5 with aqueous $\mathrm{NH}_{4} \mathrm{OH}$ solution. The organic layer was separated and the aqueous phase was further extracted with fresh $\mathrm{CH}_{2} \mathrm{Cl}_{2}(2 \times 100 \mathrm{~mL})$. The combined organic phases were dried with $\mathrm{Na}_{2} \mathrm{SO}_{4}$, filtered, and evaporated to dryness. The crude product was purified by column chromatography on silica gel $\left(\mathrm{CH}_{2} \mathrm{Cl}_{2} / \mathrm{MeOH}, 100: 0 \rightarrow 98.5: 1.5\right)$ to give 2,2'-(4-(hexyloxy)pyridine-2,6-diyl)bis(1-isopentyl-1H-benzo[d]imidazole) (L3, $1.33 \mathrm{~g}, 2.41$ mmol yield $76 \%$ ).

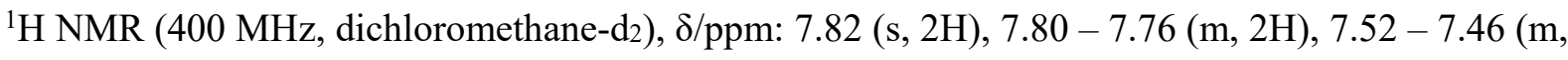
2H), $7.39-7.27(\mathrm{~m}, 4 \mathrm{H}), 4.79-4.71(\mathrm{~m}, 4 \mathrm{H}), 4.25\left(\mathrm{t},{ }^{3} \mathrm{~J}=6.5 \mathrm{~Hz}, 2 \mathrm{H}\right), 1.97-1.82(\mathrm{~m}, 2 \mathrm{H}), 1.71-$ $1.58(\mathrm{~m}, 4 \mathrm{H}), 1.67-1.58(\mathrm{~m}, 4 \mathrm{H}), 1.48-1.30(\mathrm{~m}, 6 \mathrm{H}), 1.05-0.88(\mathrm{~m}, 3 \mathrm{H}), 0.71\left(\mathrm{~d},{ }^{3} J=6.6 \mathrm{~Hz}\right.$, 12H). ESI-MS $\left(\mathrm{CH}_{2} \mathrm{Cl}_{2}\right): m / z: 552.8\left([\mathrm{M}+\mathrm{H}]^{+}\right)$.

\section{Preparation of 6-(ethoxycarbonyl)-4-(hexyloxy) picolinic acid (11)}

4-(hexyloxy)pyridine-2,6-dicarboxylate (7, $6 \mathrm{~g}, 18.5 \mathrm{mmol})$ was dissolved in ethanol $(250 \mathrm{~mL})$ and cooled in an ice bath. A solution of potassium hydroxide $(830 \mathrm{mg}, 14.8 \mathrm{mmol})$ in ethanol $(50 \mathrm{~mL})$ was dropwise added (30 $\mathrm{min})$. The resulting solution was stirred at RT for $2 \mathrm{~h}$. Reaction progress was

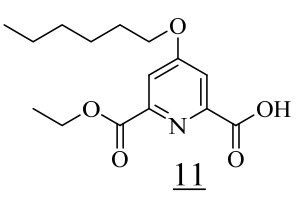
checked by TLC (silica plate, $\mathrm{CH}_{2} \mathrm{Cl}_{2} / \mathrm{MeOH} 97 / 3 \mathrm{v} / \mathrm{v}$ ). After disappearance of 7, the solution was 
neutralized with $\mathrm{HCl} \mathrm{10 \%} \mathrm{and} \mathrm{the} \mathrm{solvent} \mathrm{evaporated.} \mathrm{The} \mathrm{resulting} \mathrm{residue} \mathrm{was} \mathrm{dissolved} \mathrm{in} \mathrm{water}$ $(100 \mathrm{~mL})$ and $\mathrm{pH}$ adjusted to $9-10$ then aqueous phase was extracted with diethylether $(2 \times 50 \mathrm{~mL})$. The combined organic phase contained some unreacted starting material was discarded. The $\mathrm{pH}$ of the aqueous phase was then adjusted to $1.0(\mathrm{HCl} \mathrm{10 \% )} \mathrm{and} \mathrm{a} \mathrm{second} \mathrm{extraction} \mathrm{process} \mathrm{was} \mathrm{performed}$ with dichloromethane $(3 \times 100 \mathrm{~mL})$. The combined dichloromethane phase was dried over $\mathrm{Na}_{2} \mathrm{SO}_{4}$, filtered, and evaporated to give 6-(ethoxycarbonyl)-4-(hexyloxy) picolinic acid (11, 3.9 g, $13.2 \mathrm{mmol}$, yield $72 \%$ ).

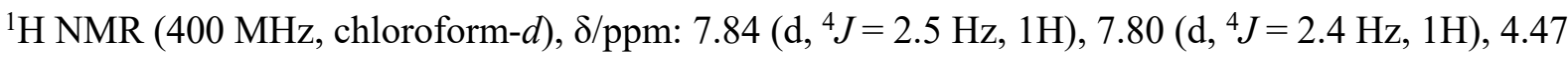
$\left(\mathrm{q},{ }^{3} J=7.1 \mathrm{~Hz}, 2 \mathrm{H}\right), 4.15\left(\mathrm{t},{ }^{3} J=6.5 \mathrm{~Hz}, 2 \mathrm{H}\right), 1.80-1.89(\mathrm{~m}, 2 \mathrm{H}), 1.41-1.52(\mathrm{~m}, 5 \mathrm{H}), 1.31-1.38$ (m, 4H), $0.92\left(\mathrm{t},{ }^{3} J=7.1 \mathrm{~Hz}, 3 \mathrm{H}\right)$; ESI-MS (positive mode/ $\left.\mathrm{CH}_{2} \mathrm{Cl}_{2}\right), m / z: 296.5\left([\mathrm{M}+\mathrm{H}]^{+}\right)$.

\section{Preparation of ethyl 4-(hexyloxy)-6-(isopentyl (2-nitrophenyl)carbamoyl) picolinate (12)}

6-(ethoxycarbonyl)-4-(hexyloxy)picolinic acid (11, $3 \mathrm{~g}, 10.16 \mathrm{mmol})$ and DMF $(30 \mu \mathrm{L})$ were heated to reflux in thionyl chloride $(7.5 \mathrm{~mL}, 101$ mmol) for $1 \mathrm{~h}$. Excess thionyl chloride was distilled from the reaction mixture, which was then co-evaporated with dry $\mathrm{CH}_{2} \mathrm{Cl}_{2}(3 \times 20 \mathrm{~mL})$ and

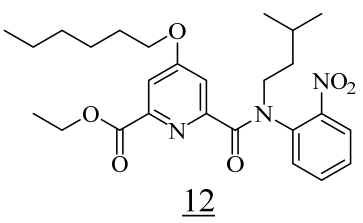
dried under vacuum. The solid was re-dissolved in freshly distilled $\mathrm{CH}_{2} \mathrm{Cl}_{2}(30 \mathrm{~mL})$ and a solution of $\mathrm{N}$-isopentyl-2-nitroaniline (1a, $\left.2.22 \mathrm{~g}, 10.66 \mathrm{mmol}\right)$ in $\mathrm{CH}_{2} \mathrm{Cl}_{2}(20$ $\mathrm{mL}$ ) was slowly added under an inert atmosphere. The resulting mixture was heated to reflux for 24 $\mathrm{h}$ and the $\mathrm{pH}$ value was kept close to $\mathrm{pH} 9$ by adding small amounts of $N, N$-diisopropylethylamine. The mixture was partitioned between $\mathrm{CH}_{2} \mathrm{Cl}_{2}(60 \mathrm{~mL})$ and half-saturated aqueous $\mathrm{NH}_{4} \mathrm{Cl}(60 \mathrm{~mL})$. The organic layer was separated and the aqueous phase was further extracted with fresh $\mathrm{CH}_{2} \mathrm{Cl}_{2}(2 \times 50$ $\mathrm{mL}$ ). The combined organic phases were dried with $\mathrm{Na}_{2} \mathrm{SO}_{4}$, filtered and evaporated to dryness. The crude product was purified by column chromatography on silica gel $\left(\mathrm{CH}_{2} \mathrm{Cl}_{2} / \mathrm{MeOH}, 100: 0 \rightarrow 99: 1\right)$ to give compound ethyl 4-(hexyloxy)-6-(isopentyl (2-nitrophenyl)carbamoyl) picolinate as a yellow powder. (12, $3.94 \mathrm{~g}, 8.11 \mathrm{mmol}$, yield 80\%).

${ }^{1} \mathrm{H}$ NMR (400 MHz, Chloroform-d, major rotamer), $\delta / \mathrm{ppm}: 7.95$ (dd, $\left.{ }^{3} J=8.2,{ }^{4} J=1.5 \mathrm{~Hz}, 1 \mathrm{H}\right), 7.62$ - $7.48(\mathrm{~m}, 2 \mathrm{H}), 7.47-7.31(\mathrm{~m}, 3 \mathrm{H}), 4.35-4.08(\mathrm{~m}, 3 \mathrm{H}), 4.03\left(\mathrm{t},{ }^{3} \mathrm{~J}=6.6 \mathrm{~Hz}, 2 \mathrm{H}\right), 3.7-3.6(\mathrm{~m}, 1 \mathrm{H})$, $1.84-1.73(\mathrm{~m}, 2 \mathrm{H}), 1.67-1.51(\mathrm{~m}, 3 \mathrm{H}), 1.48-1.38(\mathrm{~m}, 3 \mathrm{H}), 1.36-1.26(\mathrm{~m}, 7 \mathrm{H}), 0.95-0.86(\mathrm{~m}$, 9H); ESI-MS $\left(\mathrm{CH}_{2} \mathrm{Cl}_{2}\right), m / z: 486.3\left([\mathrm{M}+\mathrm{H}]^{+}\right)$.

\section{Preparation of 4-(hexyloxy)-6-(isopentyl (2-nitrophenyl) carbamoyl) picolinic acid (13)}

A solution of $\mathrm{LiOH} \cdot \mathrm{H}_{2} \mathrm{O}(1.99 \mathrm{~g}, 47.5 \mathrm{mmol})$ in methanol:water $(80 \mathrm{~mL}: 20$ $\mathrm{mL})$ was dropwise added to a cooled $\left(0^{\circ} \mathrm{C}\right)$ solution of $(12,4.61 \mathrm{~g}, 9.5$ $\mathrm{mmol})$ in THF $(30 \mathrm{~mL})$. The mixture was stirred for about $3 \mathrm{~h}$ at $0{ }^{\circ} \mathrm{C}$, and the reaction progress was followed by TLC (silica plate, $\mathrm{CH}_{2} \mathrm{Cl}_{2} / \mathrm{MeOH}$

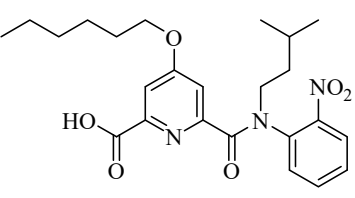

$\underline{13}$ $97 / 4 \mathrm{v} / \mathrm{v}$ ). After completion of the reaction, the mixture was neutralized 
with $\mathrm{HCl} 10 \%$ and evaporated under vacuum to remove THF and methanol. The resulting mixture was extracted with dichloromethane $(3 \times 100 \mathrm{~mL})$. The combined organic phases were dried with $\mathrm{Na}_{2} \mathrm{SO}_{4}$, filtered, and evaporated to dryness. The crude product was purified by column chromatography on silica gel $\left(\mathrm{CH}_{2} \mathrm{Cl}_{2} / \mathrm{MeOH}, 99.5: 0.5 \rightarrow 97: 3\right)$ to give 4-(hexyloxy)-6-(isopentyl (2nitrophenyl) carbamoyl) picolinic acid as yellow crystals (13, $3.13 \mathrm{~g}, 6.84 \mathrm{mmol}$, yield 72\%).

${ }^{1} \mathrm{H}$ NMR (400 MHz, chloroform-d, major rotamer), $\delta /$ ppm: $9.61(\mathrm{~s}, 1 \mathrm{H}), 7.87\left(\mathrm{dd},{ }^{3} \mathrm{~J}=8.2,{ }^{4} \mathrm{~J}=1.5\right.$ $\mathrm{Hz}, 1 \mathrm{H}), 7.66-7.57(\mathrm{~m}, 2 \mathrm{H}), 7.53\left(\mathrm{~d},{ }^{3} J=2.5 \mathrm{~Hz}, 1 \mathrm{H}\right), 7.46\left(\mathrm{td},{ }^{3} J=7.8,{ }^{4} \mathrm{~J}=1.4 \mathrm{~Hz}, 1 \mathrm{H}\right), 7.37(\mathrm{dd}$, $\left.{ }^{3} J=7.9,{ }^{4} J=1.4 \mathrm{~Hz}, 1 \mathrm{H}\right), 4.20-4.11(\mathrm{~m}, 1 \mathrm{H}), 4.07\left(\mathrm{t},{ }^{3} J=6.6 \mathrm{~Hz}, 2 \mathrm{H}\right), 3.73-3.63(\mathrm{~m}, 1 \mathrm{H}), 1.87-$ $1.72(\mathrm{~m}, 2 \mathrm{H}), 1.70-1.50(\mathrm{~m}, 3 \mathrm{H}), 1.49-1.38(\mathrm{~m}, 2 \mathrm{H}), 1.38-1.27(\mathrm{~m}, 4 \mathrm{H}), 0.98-0.88(\mathrm{~m}, 9 \mathrm{H})$. ESI-MS $\left(\mathrm{CH}_{2} \mathrm{Cl}_{2}\right), m / z: 458.8\left([\mathrm{M}+\mathrm{H}]^{+}\right)$.

\section{Preparation of N2-(4-bromo-2-nitrophenyl)-4-(hexyloxy)-N2, N6-diisopentyl-N6-(2- nitrophenyl)pyridine-2,6-dicarboxamide (14)}

4-(hexyloxy)-6-(isopentyl (2-nitrophenyl) carbamoyl) picolinic acid $(\mathbf{1 3}, 3 \mathrm{~g}, 6.5 \mathrm{mmol})$ and $\mathrm{DMF}(30 \mu \mathrm{L})$ were heated to reflux in thionyl chloride $(4.7 \mathrm{~mL}, 65 \mathrm{mmol})$ for $1 \mathrm{~h}$. Excess thionyl chloride was distilled from the reaction mixture, which was then co-evaporated with dry $\mathrm{CH}_{2} \mathrm{Cl}_{2}(3 \times 15 \mathrm{~mL})$ and dried under vacuum. The solid was

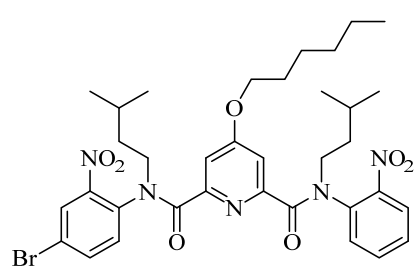

$\underline{14}$ re-dissolved in freshly distilled $\mathrm{CH}_{2} \mathrm{Cl}_{2}(25 \mathrm{~mL})$ and a solution of 4bromo-N-isopentyl-2-nitroaniline $(\mathbf{1 b}, 1.91 \mathrm{~g}, 7.15 \mathrm{mmol})$ in $\mathrm{CH}_{2} \mathrm{Cl}_{2}(15 \mathrm{~mL})$ was slowly added under an inert atmosphere. The resulting mixture was heated to reflux for $24 \mathrm{~h}$ and the $\mathrm{pH}$ value was kept close to 9 by the addition of small amounts of $N, N$-diisopropylethylamine. The mixture was partitioned between $\mathrm{CH}_{2} \mathrm{Cl}_{2}(50 \mathrm{~mL})$ and half-saturated aqueous $\mathrm{NH}_{4} \mathrm{Cl}(50 \mathrm{~mL})$. The organic layer was separated and the aqueous phase was further extracted with fresh $\mathrm{CH}_{2} \mathrm{Cl}_{2}(2 \times 50 \mathrm{~mL})$. The combined organic phases were dried with $\mathrm{Na}_{2} \mathrm{SO}_{4}$, filtered, and evaporated to dryness. The crude product was purified by column chromatography on silica gel $\left(\mathrm{CH}_{2} \mathrm{Cl}_{2}: \mathrm{MeOH}, 100: 0 \rightarrow 99: 1\right)$ to give N2-(4-bromo-2-nitrophenyl)-4-(hexyloxy)-N2， N6-diisopentyl-N6-(2-nitrophenyl)pyridine-2,6dicarboxamide $(\mathbf{1 4}, 4.01 \mathrm{~g}, 5.5 \mathrm{mmol}$, yield $85 \%)$ as a yellowish oil.

${ }^{1} \mathrm{H}$ NMR (400 MHz, DMSO-d6, $398 \mathrm{~K}$ ), $\delta / \mathrm{ppm}: 8.14$ (s, 1H), 7.93 (t, $\left.{ }^{3} J=9.9 \mathrm{~Hz}, 2 \mathrm{H}\right), 7.72\left(\mathrm{t},{ }^{3} J=\right.$ $7.8 \mathrm{~Hz}, 1 \mathrm{H}), 7.59-7.32(\mathrm{~m}, 3 \mathrm{H}), 7.09-6.85(\mathrm{~m}, 2 \mathrm{H}), 4.13-4.01(\mathrm{~m}, 2 \mathrm{H}), 3.62(\mathrm{~s}, 4 \mathrm{H}), 1.77-1.64$ $(\mathrm{m}, 2 \mathrm{H}), 1.62-1.25(\mathrm{~m}, 12 \mathrm{H}), 0.97-0.61(\mathrm{~m}, 15 \mathrm{H})$. ESI-MS $\left(\mathrm{CH}_{2} \mathrm{Cl}_{2}\right), m / z: 727.3\left([\mathrm{M}+\mathrm{H}]^{+}\right)$. 
Preparation of 5-bromo-2-(4-(hexyloxy)-6-(1-isopentyl-1H-benzo[d]imidazol-2-yl)pyridin-2yl)-1-isopentyl-1H-benzo[d]imidazole (L4)

Sodium dithionite $(8.4 \mathrm{~g}, 48 \mathrm{mmol})$, water $(30 \mathrm{~mL})$ and methanol $(4 \mathrm{~mL})$ were mixed in a roundbottomed flask. A solution of N2-(4-bromo-2-nitrophenyl)-4(hexyloxy)-N2, N6-diisopentyl-N6-(2-nitrophenyl)pyridine-2,6dicarboxamide $(\mathbf{1 4}, 3 \mathrm{~g}, 4.1 \mathrm{mmol})$ in THF $(40 \mathrm{~mL})$ was added and the resulting mixture was stirred at room temperature for $24 \mathrm{~h}$ under an inert atmosphere. Solvent was evaporated under reduced pressure at

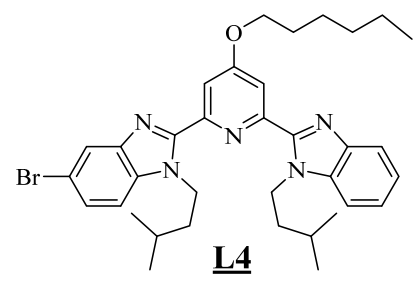
room temperature. Dichloromethane $(50 \mathrm{~mL})$ and $10 \%$ aq. $\mathrm{NaHCO}_{3}(30 \mathrm{~mL})$ were then added and the mixture stirred for $1 \mathrm{~h}$. The organic phase was separated and the aqueous phase was further extracted with fresh dichloromethane $(3 \times 100 \mathrm{~mL})$. The combined organic phases were dried with $\mathrm{Na}_{2} \mathrm{SO}_{4}$, filtered and evaporated under reduced pressure to dryness. The residue was dissolved in 30 $\mathrm{mL}$ xylene and p-toluene sulfonic acid $(0.6 \mathrm{~g}, 3.4 \mathrm{mmol})$ was added. The resulting solution was heated at $120{ }^{\circ} \mathrm{C}$ for $6 \mathrm{~h}$. The solvent was evaporated under reduced pressure to dryness and the residue partitioned between $\mathrm{CH}_{2} \mathrm{Cl}_{2}(50 \mathrm{~mL})$ and water $(30 \mathrm{~mL})$. The $\mathrm{pH}$ of the solution was adjusted to 7.5 8.5 with $10 \%$ aq. $\mathrm{K}_{2} \mathrm{CO}_{3}$. The organic phase was separated and the aqueous phase extracted with dichloromethane $(2 \times 50 \mathrm{~mL})$. The combined organic phases were dried with $\mathrm{Na}_{2} \mathrm{SO}_{4}$, filtered, and evaporated under reduced pressure to dryness. The crude product was purified by column chromatography on silica gel $\left(\mathrm{CH}_{2} \mathrm{Cl}_{2} / \mathrm{MeOH}, 99.5: 0.5 \rightarrow 98.5: 1.5\right)$ to give 5-bromo-2-(4-(hexyloxy)6-(1-isopentyl-1H-benzo[d]imidazol-2-yl)pyridin-2-yl)-1-isopentyl-1H-benzo[d]imidazole (L4, 2.32 g, 3.67 mmol, yield 90\%).

${ }^{1} \mathrm{H}$ NMR (400 MHz, chloroform-d), $\delta / \mathrm{ppm}: 7.99$ (d, $\left.{ }^{4} J=1.8 \mathrm{~Hz}, 1 \mathrm{H}\right), 7.89-7.84(\mathrm{~m}, 1 \mathrm{H}), 7.82$ (d, $\left.{ }^{4} J=2.4 \mathrm{~Hz}, 1 \mathrm{H}\right), 7.77\left(\mathrm{~d},{ }^{4} J=2.4 \mathrm{~Hz}, 1 \mathrm{H}\right), 7.50-7.41(\mathrm{~m}, 2 \mathrm{H}), 7.40-7.28(\mathrm{~m}, 3 \mathrm{H}), 4.74-4.64(\mathrm{~m}$, $4 \mathrm{H}), 4.23\left(\mathrm{t},{ }^{3} \mathrm{~J}=6.5 \mathrm{~Hz}, 2 \mathrm{H}\right), 1.93-1.76(\mathrm{~m}, 2 \mathrm{H}), 1.67-1.54(\mathrm{~m}, 4 \mathrm{H}), 1.54-1.44(\mathrm{~m}, 2 \mathrm{H}), 1.45-$ $1.30(\mathrm{~m}, 6 \mathrm{H}), 0.96-0.87(\mathrm{~m}, 3 \mathrm{H}), 0.66-0.72(\mathrm{~m}, 12 \mathrm{H})$. ESI-MS $\left(\mathrm{CH}_{2} \mathrm{Cl}_{2}\right), m / z: 631.2\left([\mathrm{M}+\mathrm{H}]^{+}\right)$.

Preparation of 2,2'-bis(4-(hexyloxy)-6-(1-isopentyl-1H-benzo[d]imidazol-2-yl)pyridin-2-yl)1,1'-diisopentyl-1H,1'H-5,5'-bibenzo[d]imidazole (L6)

Bis(cyclooctadiene)nickel(0) (1.56 g, $5.68 \mathrm{mmol})$, cyclooctadiene $(0.69 \mathrm{~mL}, 5.68 \mathrm{mmol})$, and 2,2`bipyridine ( $891 \mathrm{mg}, 5.7 \mathrm{mmol}$ ) were dissolved in dry toluene $(22 \mathrm{~mL})$ and dry $N, N$-dimethylformamide $(22$ $\mathrm{mL}$ ) in a Schlenk flask within a glove box. The mixture was heated at $60{ }^{\circ} \mathrm{C}$ with stirring under argon for $20 \mathrm{~min}$ to generate the catalyst. A solution of the

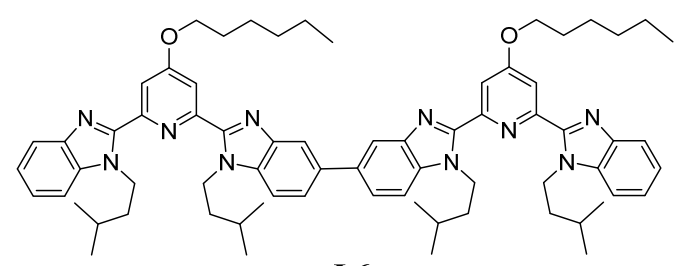
$\underline{\mathbf{L} 6}$ 5-bromo-2-(4-(hexyloxy)-6-(1-isopentyl-1Hbenzo[d]imidazol-2-yl)pyridin-2-yl)-1-isopentyl-1H-benzo[d]imidazol L2 (1.5 g, $2.3 \mathrm{mmol}$ ) in dry 
toluene $(22 \mathrm{~mL})$ was then added, and the reaction mixture was heated at $80{ }^{\circ} \mathrm{C}$ for $48 \mathrm{~h}$. The solvent was evaporated under reduced pressure and the residue partitioned between $\mathrm{CH}_{2} \mathrm{Cl}_{2}(100 \mathrm{~mL})$ and water $(50 \mathrm{~mL})$. A solution of $\mathrm{Na}_{2} \mathrm{H}_{2}$ EDTA $2 \mathrm{H}_{2} \mathrm{O}(5 \mathrm{~g})$ in water $(50 \mathrm{~mL})$ was poured into the resulting mixture, and $\mathrm{pH}$ was adjusted to 8.5 with aqueous $\mathrm{NH}_{4} \mathrm{OH}$. After being stirred for $30 \mathrm{~min}$, the organic layer was separated and the aqueous phase was further extracted with fresh $\mathrm{CH}_{2} \mathrm{Cl}_{2}(3 \times 50 \mathrm{~mL})$, the combined organic phases were dried with $\mathrm{Na}_{2} \mathrm{SO}_{4}$, evaporated to dryness, and the crude product was purified by column chromatography on silica gel $\left(\mathrm{CH}_{2} \mathrm{Cl}_{2}: \mathrm{MeOH}, 100: 0 \rightarrow 99.5: 0.5\right)$ to give $\mathbf{L 6}(0.57$ g, $0.51 \mathrm{mmol}$, yield 45\%).

${ }^{1} \mathrm{H}$ NMR (400 MHz, dichloromethane-d2), $\delta / \mathrm{ppm}: 8.12-8.07$ (m, 2H), $7.86\left(\mathrm{~d},{ }^{4} \mathrm{~J}=2.4 \mathrm{~Hz}, 2 \mathrm{H}\right), 7.83$ $\left(\mathrm{d},{ }^{4} J=2.4 \mathrm{~Hz}, 2 \mathrm{H}\right), 7.84-7.81(\mathrm{~m}, 2 \mathrm{H}), 7.72\left(\mathrm{dd},{ }^{3} J=8.5,{ }^{4} J=1.7 \mathrm{~Hz}, 2 \mathrm{H}\right), 7.60-7.54(\mathrm{~m}, 2 \mathrm{H})$, $7.52-7.45(\mathrm{~m}, 2 \mathrm{H}), 7.39-7.26(\mathrm{~m}, 4 \mathrm{H}), 4.86-4.70(\mathrm{~m}, 8 \mathrm{H}), 4.26\left(\mathrm{t},{ }^{3} J=6.5 \mathrm{~Hz}, 4 \mathrm{H}\right), 1.97-1.81$ (m, 4H), $1.74-1.58(\mathrm{~m}, 8 \mathrm{H}), 1.57-1.49(\mathrm{~m}, 4 \mathrm{H}), 1.48-1.32(\mathrm{~m}, 12 \mathrm{H}), 0.98-0.88(\mathrm{~m}, 6 \mathrm{H}), 0.73$ $\left(\mathrm{d},{ }^{3} \mathrm{~J}=6.6 \mathrm{~Hz}, 24 \mathrm{H}\right)$. ESI-MS $\left(\mathrm{CH}_{2} \mathrm{Cl}_{2}\right), m / z: 1102.1\left([\mathrm{M}+\mathrm{H}]^{+}\right) ; 551.3\left([\mathrm{M}+2 \mathrm{H}]^{2+}\right)$.

Preparation of 2,2'-(4-(hexyloxy)pyridine-2,6-diyl)bis(1-isopentyl-5-(4,4,5,5-tetramethyl-1,3,2dioxaborolan-2-yl)-1H-benzo[d]imidazole) (15).

L1 (900 mg, $1.26 \mathrm{mmol}$ ), bis(pinacolato)diboron (768 mg, $3.03 \mathrm{mmol}$ ), KOAc (346 $\mathrm{mg}, 3.53 \mathrm{mmol})$, and [1,1'Bis(diphenylphosphino)ferrocene]palladium(II) dichloride (29 mg, $0.04 \mathrm{mmol}$ ) were mixed in $\mathrm{CH}_{2} \mathrm{Cl}_{2}(20 \mathrm{~mL})$ under an argon atmosphere. Degassed DMF $(10 \mathrm{~mL})$ was added

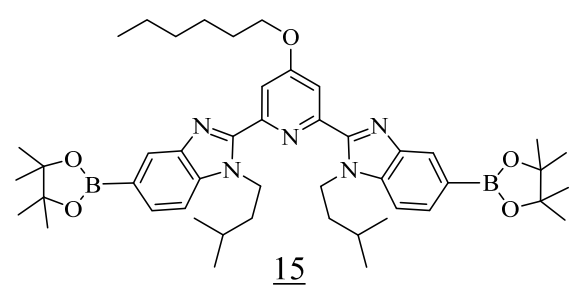
and the mixture was heated at $80^{\circ} \mathrm{C}$ for $72 \mathrm{~h}$. Half sat. $\mathrm{NaCl}(150 \mathrm{~mL})$ was added and the aqueous phase was extracted with diethyl ether $(4 \times 100 \mathrm{~mL})$. The combined organic phases were dried with $\mathrm{Na}_{2} \mathrm{SO}_{4}$, filtered, evaporated to dryness, and the crude product was purified by column chromatography on silica gel $\left(\mathrm{CH}_{2} \mathrm{Cl}_{2}: \mathrm{MeOH}, 99: 1\right)$ to give 13 (370 mg, $0.46 \mathrm{mmol}$, yield 30\%).

${ }^{1} \mathrm{H}$ NMR (400 MHz, dichloromethane-d 2 ), $\delta / \mathrm{ppm}: 8.21$ (s, 2H), $7.84(\mathrm{~s}, 2 \mathrm{H}), 7.75\left(\mathrm{dd},{ }^{3} \mathrm{~J}=8.2,{ }^{4} \mathrm{~J}=\right.$ $1.0 \mathrm{~Hz}, 2 \mathrm{H}), 7.48\left(\mathrm{dd},{ }^{3} J=8.2,{ }^{4} \mathrm{~J}=1 \mathrm{~Hz}, 2 \mathrm{H}\right), 4.81-4.66(\mathrm{~m}, 4 \mathrm{H}), 4.27\left(\mathrm{t},{ }^{3} \mathrm{~J}=6.5 \mathrm{~Hz}, 2 \mathrm{H}\right), 1.94-$ $1.85(\mathrm{~m}, 2 \mathrm{H}), 1.67-1.57(\mathrm{~m}, 4 \mathrm{H}), 1.57-1.48(\mathrm{~m}, 2 \mathrm{H}), 1.44-1.32(\mathrm{~m}, 30 \mathrm{H}), 0.96-0.91(\mathrm{~m}, 3 \mathrm{H})$, $0.69\left(\mathrm{~d},{ }^{3} J=6.7 \mathrm{~Hz}, 12 \mathrm{H}\right)$. ESI-MS $\left(\mathrm{CH}_{2} \mathrm{Cl}_{2}\right), m / z: 804.5\left([\mathrm{M}+\mathrm{H}]^{+}\right)$.

Preparation of 2',2'"-(4-(hexyloxy)pyridine-2,6-diyl)bis(2-(4-(hexyloxy)-6-(1-isopentyl-1Hbenzo[d]imidazol-2-yl)pyridin-2-yl)-1,1'-diisopentyl-1 H,1'H-5,5'-bibenzo[d]imidazole) (L7)

A solution of 2,2'-(4-(hexyloxy)pyridine-2,6-diyl)bis(1-isopentyl-5-(4,4,5,5-tetramethyl-1,3,2dioxaborolan-2-yl)-1H-benzo[d]imidazole $(\mathbf{1 5}, 300 \mathrm{mg}, 0.37 \mathrm{mmol})$ in dioxane $(30 \mathrm{~mL})$ was introduced into a Schlenk tube containing $\mathbf{L 2}(540 \mathrm{mg}, 0.85 \mathrm{mmol})$, bis(triphenylphosphine)palladium(II) dichloride ( $82 \mathrm{mg}, 0.1 \mathrm{mmol}, 0.2$ equiv) and $\mathrm{K}_{2} \mathrm{CO}_{3}(181 \mathrm{mg}$, 
$1.85 \mathrm{mmol}$ ) under an inert atmosphere. Water $(1.3 \mathrm{~mL})$ was added into the Schlenk and the mixture was heated at reflux for $24 \mathrm{~h}$ under argon.

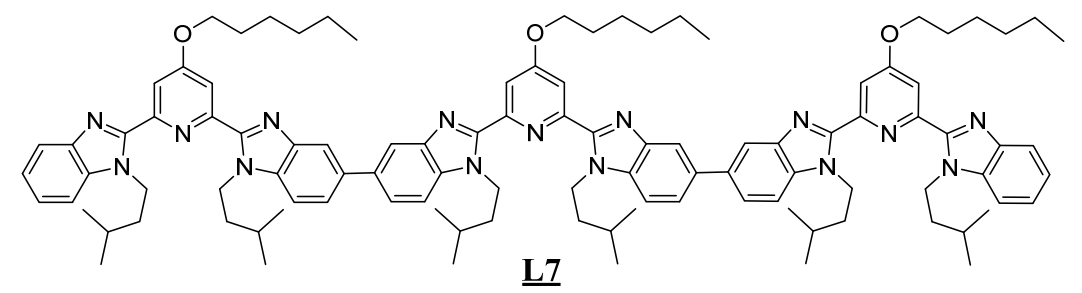
After evaporation to dryness, the residue was partitioned between half sat. $\mathrm{NaHCO}_{3}(100 \mathrm{~mL})$ and dichloromethane $(100 \mathrm{~mL})$. The organic layer was separated and the aqueous phase was further extracted with fresh $\mathrm{CH}_{2} \mathrm{Cl}_{2}(3 \times 100 \mathrm{~mL})$, the combined organic phases were dried with $\mathrm{Na}_{2} \mathrm{SO}_{4}$, evaporated to dryness, and the crude product was purified by column chromatography on silica gel $\left(\mathrm{CH}_{2} \mathrm{Cl}_{2}: \mathrm{MeOH}, 99: 1 \rightarrow 98: 2\right)$ to give $\mathbf{L} 7$ (365 mg, $0.22 \mathrm{mmol}$, yield 60\%).

${ }^{1} \mathrm{H}$ NMR (400 MHz, chloroform- $d$ ), $\delta / \mathrm{ppm}: 8.17$ (s, 4H), $7.90-7.85(\mathrm{~m}, 6 \mathrm{H}), 7.83\left(\mathrm{~d},{ }^{4} J=2.4 \mathrm{~Hz}\right.$, 2H), $7.76-7.68(\mathrm{~m}, 4 \mathrm{H}), 7.58-7.52(\mathrm{~m}, 4 \mathrm{H}), 7.50-7.43(\mathrm{~m}, 2 \mathrm{H}), 7.41-7.30(\mathrm{~m}, 4 \mathrm{H}), 4.84-4.69$ $(\mathrm{m}, 12 \mathrm{H}), 4.30-4.22(\mathrm{~m}, 6 \mathrm{H}), 1.97-1.81(\mathrm{~m}, 6 \mathrm{H}), 1.73-1.59(\mathrm{~m}, 12 \mathrm{H}), 1.57-1.30(\mathrm{~m}, 6 \mathrm{H}), 0.99$ $-0.85(\mathrm{~m}, 9 \mathrm{H}), 0.81-0.68(\mathrm{~m}, 36 \mathrm{H})$. ESI-MS $\left(\mathrm{CH}_{2} \mathrm{Cl}_{2}\right), m / z: 551.0\left([\mathrm{M}+3 \mathrm{H}]^{3+}\right), 826.3\left([\mathrm{M}+2 \mathrm{H}]^{2+}\right)$, $1652.8\left([\mathrm{M}+\mathrm{H}]^{+}\right)$.

\section{Preparation of $\left[\operatorname{LnLk}(\mathrm{hfa})_{3}\right]$ complexes $(\mathrm{Ln}=\mathrm{La}, \mathrm{Eu}$ and $k=3,4,5)$}

Stoichiometric amounts of $\mathbf{L} \boldsymbol{k}$ and [ $\operatorname{Ln}(\mathrm{hfa})_{3} \mathrm{dig}$ ] were reacted in acetonitrile/dichloromethane (2:1). Subsequent slow evaporation of the saturated solutions provided X-ray quality crystals of [LnLk(hfa) $)_{3}$ (Tables S8-S39 and Figures 4 and S3-S9).

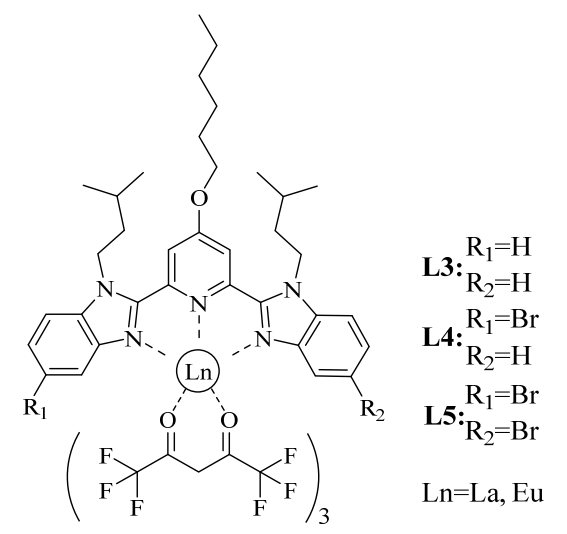

[LaL3(hfa)3]: ${ }^{1} \mathrm{H}$ NMR (400 MHz, $\mathrm{CD}_{2} \mathrm{Cl}_{2}$ ), $\delta / \mathrm{ppm}: 8.03$ (d, $\left.J=8.3 \mathrm{~Hz}, 2 \mathrm{H}\right), 7.57$ (d, $J=8.3 \mathrm{~Hz}$, 2H), 7.53-7.45 (m, 4H), $7.39(\mathrm{ddd}, J=8.3,7.1,1.2 \mathrm{~Hz}, 2 \mathrm{H}), 5.89(\mathrm{~s}, 3 \mathrm{H}), 4.62-4.53(\mathrm{~m}, 4 \mathrm{H}), 4.32$ $(\mathrm{t}, J=6.5 \mathrm{~Hz}, 2 \mathrm{H}), 2.06-1.95(\mathrm{~m}, 6 \mathrm{H}), 1.88(\mathrm{dq}, J=13.3,6.6 \mathrm{~Hz}, 2 \mathrm{H}), 1.54-1.61(\mathrm{~m}, 2 \mathrm{H}), 1.39-1.46$ (m, 4H), $1.12(\mathrm{~d}, J=6.6 \mathrm{~Hz}, 12 \mathrm{H}), 1.00-0.94(\mathrm{~m}, 3 \mathrm{H})$.

[EuL3(hfa) $)_{3}$ : ${ }^{1} \mathrm{H}$ NMR (400 MHz, $\mathrm{CD}_{2} \mathrm{Cl}_{2}$ ), $\delta / \mathrm{ppm}: 22.59$ (s, 2H), $11.20-11.28$ (m, 2H), 10.03 (dd, $J=8.3,7.0 \mathrm{~Hz}, 2 \mathrm{H}), 8.94(\mathrm{~d}, J=8.4 \mathrm{~Hz}, 2 \mathrm{H}), 5.86(\mathrm{~s}, 2 \mathrm{H}), 5.78-5.66(\mathrm{~m}, 4 \mathrm{H}), 3.98(\mathrm{t}, J=6.6 \mathrm{~Hz}$, $2 \mathrm{H}), 3.07-2.94(\mathrm{~m}, 4 \mathrm{H}), 2.40-2.34(\mathrm{~m}, 2 \mathrm{H}), 1.63(\mathrm{q}, J=7.0 \mathrm{~Hz}, 2 \mathrm{H}), 1.36(\mathrm{~d}, J=6.6 \mathrm{~Hz}, 12 \mathrm{H})$, $1.31-1.25(\mathrm{~m}, 2 \mathrm{H}), 1.17-1.22(\mathrm{~m}, 4 \mathrm{H}), 0.83-0.75(\mathrm{~m}, 3 \mathrm{H})$. 
[EuL4(hfa) $)_{3}$ : ${ }^{1} \mathrm{H}$ NMR (400 MHz, $\mathrm{CD}_{2} \mathrm{Cl}_{2}$ ), $\delta / \mathrm{ppm}: 23.59$ (s, 1H), 22.69 (s, 1H), 11.51 (t, J=6.2 Hz, 1H), $10.21-10.12$ (m, 2H), 9.04 (d, $J=8.8 \mathrm{~Hz}, 1 \mathrm{H}), 8.99$ (d, $J=8.5 \mathrm{~Hz}, 1 \mathrm{H}), 6.07$ (s, 1H), $5.99-$ $5.91(\mathrm{~m}, 2 \mathrm{H}), 5.80(\mathrm{~s}, 1 \mathrm{H}), 5.68-5.59(\mathrm{~m}, 2 \mathrm{H}), 4.01(\mathrm{t}, J=6.5 \mathrm{~Hz}, 2 \mathrm{H}), 3.20-3.11(\mathrm{~m}, 2 \mathrm{H}), 2.96-$ $2.86(\mathrm{~m}, 2 \mathrm{H}), 2.28-2.9(\mathrm{~m}, 4 \mathrm{H}), 1.70-1.62(\mathrm{~m}, 2 \mathrm{H}), 1.42(\mathrm{~d}, J=6.6 \mathrm{~Hz}, 6 \mathrm{H}), 1.34(\mathrm{~d}, J=6.6 \mathrm{~Hz}$, $6 \mathrm{H}), 1.31-1.16(\mathrm{~m}, 6 \mathrm{H}), 0.86-0.79(\mathrm{~m}, 3 \mathrm{H})$.

[EuL5(hfa) ${ }_{3}$ ]: ${ }^{1} \mathrm{H}$ NMR (400 MHz, $\mathrm{CD}_{2} \mathrm{Cl}_{2}$ ), $\delta / \mathrm{ppm}: 24.49$ (s, 2H), 10.42 (d, J=8.7 Hz, 2H), 9.19 (d, $J=8.8 \mathrm{~Hz}, 2 \mathrm{H}), 5.95-5.79(\mathrm{~m}, 6 \mathrm{H}), 3.96(\mathrm{t}, J=6.6 \mathrm{~Hz}, 2 \mathrm{H}), 3.16-3.04(\mathrm{~m}, 4 \mathrm{H}), 2.36-2.45(\mathrm{~m}$, $2 \mathrm{H}), 1.58-1.66(\mathrm{~m}, 2 \mathrm{H}), 1.39(\mathrm{~d}, J=6.6 \mathrm{~Hz}, 12 \mathrm{H}), 1.30-1.17(\mathrm{~m}, 6 \mathrm{H}), 0.85-0.75(\mathrm{~m}, 3 \mathrm{H})$.

Elemental analyses are collected in Table S1.

\section{Preparation of $\left[\operatorname{L6}\left(\operatorname{Ln}(h f a)_{3}\right)_{2}\right]$ complexes $(\operatorname{Ln}=\mathrm{La}, \mathrm{Eu}, \mathrm{Yb}, \mathrm{Lu})$}

Two equivalents of [ $\left.\mathrm{Ln}(\mathrm{hfa})_{3} \operatorname{dig}\right](\mathrm{Ln}=\mathrm{La}, \mathrm{Eu}, \mathrm{Yb}, \mathrm{Lu})$ were dissolved in benzene and mixed with a solution of one equivalents of L6 in benzene. Slow evaporation of the saturated solutions provided X-ray quality crystals of $\left[\mathbf{L 6}\left(\operatorname{Ln}(\mathrm{hfa})_{3}\right)_{2}\right](\mathrm{Ln}=\mathrm{La}, \mathrm{Eu}, \mathrm{Yb}, \mathrm{Lu})$

[L6(Eu(hfa) $\left.)_{2}\right)_{2}$ : ${ }^{1} \mathrm{H}$ NMR (400 MHz, $\mathrm{CD}_{2} \mathrm{Cl}_{2}$ ), $\delta / \mathrm{ppm}: 24.13$ (s, 2H), 21.10 (s, 2H), 13.48 (d, J=8.4 $\mathrm{Hz}, 2 \mathrm{H}), 10.87$ (d, $J=6.7 \mathrm{~Hz}, 2 \mathrm{H}), 9.83$ (dd, $J=8.4,7.0 \mathrm{~Hz}, 2 \mathrm{H}), 9.60$ (d, $J=8.4 \mathrm{~Hz}, 2 \mathrm{H}), 8.85$ (d, $J=8.2 \mathrm{~Hz}, 2 \mathrm{H}), 6.56(\mathrm{~s}, 2 \mathrm{H}), 6.37(\mathrm{~s}, 2 \mathrm{H}), 5.91-6.04(\mathrm{~m}, 8 \mathrm{H}), 4.31(\mathrm{t}, J=6.5 \mathrm{~Hz}, 4 \mathrm{H}), 3.13-3.28$ $(\mathrm{m}, 8 \mathrm{H}), 2.43-2.58(\mathrm{~m}, 8 \mathrm{H}), 1.81-1.89(\mathrm{~m}, 4 \mathrm{H}), 1.49(\mathrm{~d}, J=6.6 \mathrm{~Hz}, 12 \mathrm{H}), 1.44(\mathrm{~d}, J=6.6 \mathrm{~Hz}$, $12 \mathrm{H}), 1.27-1.37(\mathrm{~m}, 8 \mathrm{H}), 0.85-0.92(\mathrm{~m}, 6 \mathrm{H})$.

[L6(La(hfa) $)_{2}$ ]: ${ }^{1} \mathrm{H}$ NMR (400 MHz, $\mathrm{CD}_{2} \mathrm{Cl}_{2}$ ), $\delta / \mathrm{ppm}: 8.25$ (d, $\left.J=1.6 \mathrm{~Hz}, 2 \mathrm{H}\right), 7.98(\mathrm{dt}, J=8.3,0.9$ $\mathrm{Hz}, 2 \mathrm{H}), 7.81(\mathrm{dd}, J=8.7,1.7 \mathrm{~Hz}, 2 \mathrm{H}), 7.51-7.58(\mathrm{~m}, 4 \mathrm{H}), 7.43-7.50(\mathrm{~m}, 6 \mathrm{H}), 7.31-7.36(\mathrm{~m}$, 2H), $5.86(\mathrm{~s}, 6 \mathrm{H}), 4.51-4.64(\mathrm{~m}, 8 \mathrm{H}), 4.30(\mathrm{t}, J=6.5 \mathrm{~Hz}, 4 \mathrm{H}), 1.94-2.04(\mathrm{~m}, 12 \mathrm{H}), 1.80-1.90(\mathrm{~m}$, $4 \mathrm{H}), 1.52-1.58(\mathrm{~m}, 4 \mathrm{H}), 1.36-1.46(\mathrm{~m}, 8 \mathrm{H}), 1.07-1.12(\mathrm{~m}, 24 \mathrm{H}), 0.92-0.98(\mathrm{~m}, 6 \mathrm{H})$.

Elemental analyses are collected in Table S1.

\section{Preparation of $\left[\mathrm{L} 7\left(\mathrm{Eu}(\mathrm{hfa})_{3}\right)_{3}\right]$ complex}

Three equivalents of $\left[\mathrm{Eu}(\mathrm{hfa})_{3} \mathrm{dig}\right]$ dissolved in benzene and mixed with solution of one equivalent of $\mathbf{L} 7$ in benzene. Slow evaporation of the saturated solution provided X-ray quality crystals of $\left[\mathbf{L} 7\left(\mathrm{Eu}(\mathrm{hfa})_{3}\right)_{3}\right]$.

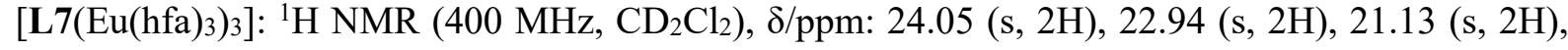
$13.34(\mathrm{~d}, J=8.6 \mathrm{~Hz}, 2 \mathrm{H}), 13.27$ (d, $J=8.5 \mathrm{~Hz}, 2 \mathrm{H}), 10.82-10.91(\mathrm{~m}, 2 \mathrm{H}), 9.83$ (dd, $J=8.4,7.0 \mathrm{~Hz}$, 2H), $9.53-9.63(\mathrm{~m}, 4 \mathrm{H}), 8.85(\mathrm{~d}, J=8.4 \mathrm{~Hz}, 2 \mathrm{H}), 6.98(\mathrm{~s}, 2 \mathrm{H}), 6.54(\mathrm{~s}, 2 \mathrm{H}), 6.36(\mathrm{~s}, 2 \mathrm{H}), 6.17-$ $6.29(\mathrm{~m}, 4 \mathrm{H}), 5.90-6.05(\mathrm{~m}, 8 \mathrm{H}), 4.61$ (t, $J=6.5 \mathrm{~Hz}, 2 \mathrm{H}), 4.30(\mathrm{t}, J=6.5 \mathrm{~Hz}, 4 \mathrm{H}), 3.12-3.67$ (m, $18 \mathrm{H}), 2.67(\mathrm{~d}, J=6.6 \mathrm{~Hz}, 2 \mathrm{H}), 2.51(\mathrm{td}, J=6.6,2.1 \mathrm{~Hz}, 4 \mathrm{H}), 2.10-2.03(\mathrm{~m}, 2 \mathrm{H}), 1.84(\mathrm{dd}, J=8.3$, $6.5 \mathrm{~Hz}, 4 \mathrm{H}), 1.58(\mathrm{~d}, J=6.7 \mathrm{~Hz}, 12 \mathrm{H}), 1.40-1.50(\mathrm{~m}, 24 \mathrm{H}), 1.30-1.35(\mathrm{~m}, 12 \mathrm{H}), 0.95-0.99$ (m, $3 \mathrm{H}), 0.84-0.89(\mathrm{~m}, 6 \mathrm{H})$. 
[L7(La(hfa) $\left.\left.)_{3}\right)_{3}\right]:{ }^{1} \mathrm{H}$ NMR (400 MHz, $\left.\mathrm{CD}_{2} \mathrm{Cl}_{2}\right), \delta / \mathrm{ppm}: 8.27-8.18(\mathrm{~m}, 4 \mathrm{H}), 7.98(\mathrm{~d}, J=8.3 \mathrm{~Hz}, 2 \mathrm{H})$, $7.80(\mathrm{dd}, J=8.7,1.7 \mathrm{~Hz}, 4 \mathrm{H}), 7.59-7.41(\mathrm{~m}, 14 \mathrm{H}), 7.36-7.30(\mathrm{~m}, 2 \mathrm{H}), 4.65-4.48(\mathrm{~m}, 12 \mathrm{H}), 4.33$ - 4.25 (m, 6H), 2.09 - $1.75(\mathrm{~m}, 30 \mathrm{H}), 1.46-1.35$ (m, 12H), 1.15 - 1.04 (m, 36H), 0.96 - 0.91 (m, 9H). The very limited amount of isolated crystals (less than $2 \mathrm{mg}$ ) was not compatible with the determination of elemental analysis.

\section{NMR and analytic measurements}

${ }^{1} \mathrm{H},{ }^{19} \mathrm{~F}$ and ${ }^{13} \mathrm{C}$ NMR spectra were recorded at $293 \mathrm{~K}$ on a Bruker Avance $400 \mathrm{MHz}$ spectrometer. Chemical shifts are given in ppm with respect to tetramethylsilane (TMS) for ${ }^{1} \mathrm{H}$ and ${ }^{13} \mathrm{C}$ NMR and with respect to $\mathrm{C}_{6} \mathrm{~F}_{6}$ for ${ }^{19} \mathrm{~F}$ NMR. Spectral assignment was assisted by $2 \mathrm{D}$ NMR experiments (COSY, NOESY). Pneumatically-assisted electrospray (ESI) mass spectra were recorded on an API 150EX (AB/MDS Sciex) equipped with a Turbo Ionspray source. Elemental analyses were performed by K. L. Buchwalder from the Microchemical Laboratory of the University of Geneva.

\section{X-Ray Crystallography}

Crystals were mounted on MiTeGen kapton cryoloops with protection oil. Cell dimensions and intensities were measured at $180 \mathrm{~K}$ on an Agilent SuperNova Dual diffractometer equipped with a $\mathrm{CCD}$ Atlas detector $(\mathrm{Cu}[\mathrm{K \alpha}]$ radiation). The structures were solved by using direct methods $\left(\right.$ ShelXS $\left.^{\mathrm{S} 9}\right)$ or dualspace methods (ShelXT) $)^{\mathrm{S} 10}$. Full-matrix least-square refinements on $F^{2}$ were performed with SHELX2014 ${ }^{\mathrm{S} 10}$ and all other calculations and drawings were performed with $\mathrm{OLEX}^{\mathrm{S} 11}$ and ORTEP ${ }^{\mathrm{S} 12}$ programs. Pertinent bond lengths, bond angles and interplanar angles were collected in Tables S2-S42 together with ORTEP views and the pertinent numbering schemes gathered in Figs S2-S9. CCDC 2094716-2094729 contain the supplementary crystallographic data. These data can be obtained free of charge from the Cambridge Crystallographic Data Centre via www.ccdc.cam.ac.uk/.

\section{References}

S1 Babel, L.; Hoang, T. N. Y.; Nozary, H.; Salamanca, J.; Guénée, L.; Piguet, C., Lanthanide Loading of Luminescent Multi-Tridentate Polymers under Thermodynamic Control. Inorg. Chem. 2014, 53, 3568-3578

S2 Zaïm, A.; Nozary, H.; Guénée, L.; Besnard, C.; Lemonnier, J.-F.; Petoud, S.; Piguet, C., NHeterocyclic Tridentate Aromatic Ligands Bound to [Ln(hfa) 3 ] Units: Thermodynamic, Structural and Luminescent Properties. Chem. Eur. J. 2012, 18, 7155-7168.

S3 a) Evans, W. J.; Giarikos, D. G.; Johnston, M. A.; Greci, M. A.; Ziller, J. W., Reactivity of the Europium Hexafluoroacetylacetonate (hfa) Complex, Eu(hfa)(3)(diglyme), and Related Analogs with Potassium: Formation of the Fluoride hfa "ate" Complexes, [LnF(hfa)(3)K(diglyme)]. J. Chem. Soc. Dalton 2002, 520-526; b) Malandrino, G.; Lo Nigro, R.; Fragala, I. L.; Benelli, C., Yttrium Beta-diketonate Glyme MOCVD Precursors: Effects of 
the Polyether Length on Stabilities, Mass Transport Properties and Coordination Spheres. Eur. J. Inorg. Chem. 2004, 500-509; c) Malandrino, G.; Fragala, I. L., Lanthanide SecondGeneration Precursors for MOCVD Applications: Effects of the Metal Ionic rRadius and Polyether Length on Coordination Spheres and Mass-Transport Properties. Coord. Chem. Rev. 2006, 250, 1605-1620; d) Valore, A.; Cariati, E.; Righetto, S.; Roberto, D.; Tessore, F.; Ugo, R.; Fragalà, I. L.; Fragalà, M. E.; Malandrino, G.; De Angelis, F.; Belpassi, L.; Ledoux-Rak, I.; Thi, K.-H.; Zyss, J., Fluorinated $\beta$-Diketonate Diglyme Lanthanide Complexes as New SecondOrder Nonlinear Optical Chromophores: the Role of $f$ Electrons in the Dipolar and Octupolar Contribution to Quadratic Hyperpolarizability. J. Am. Chem. Soc. 2010, 132, 4966-4970.

S4 Terazzi, E.; Zaïm, A.; Bocquet, B.; Varin, J.; Guénée, L.; Dutronc, T.; Lemonnier, J.-F.; Floquet, S.; Cadot, E.; Heinrich, B.; Donnio, B.; Piguet, C., Implementing Liquid-Crystalline Properties in Single-Stranded Lanthanide Helicates. Eur. J. Inorg. Chem. 2013, 3323-3333.

S5 Rizeq, N.; Georgiades, S. N., Linear and Branched Pyridyl-Oxazole Oligomers: Synthesis and Circular Dichroism Detectable Effect on c-Myc G-Quadruplex Helicity. Eur. J. Org. Chem. 2016, 122-131.

S6 Ho, T. H.; Lai, C. C.; Liu, Y. H.; Peng, S. M.; Chiu, S. H., Rotaxanes Synthesized Through Sodium-Ion-Templated Clipping of Macrocycles Around Nonconjugated Amide and Urea Functionalities. Chem. Eur. J. 2014, 20, 4563-4567.

S7 Zhang, X. F.; Huang, D. G.; Chen, Y. S.; Holm, R. H., Synthesis of Binucleating Macrocycles and Their Nickel(II) Hydroxo- and Cyano-Bridged Complexes with Divalent Ions: Anatomical Variation of Ligand Features. Inorg. Chem. 2012, 51, 11017-11029.

S8 Babel, L.; Hoang, T. N. Y.; Nozary, H.; Salamanca, J.; Guénée, L.; Piguet, C., Lanthanide Loading of Luminescent Multi-Tridentate Polymers under Thermodynamic Control. Inorg. Chem. 2014, 53, 3568-3578.

S9 Sheldrick, G.M, Acta Cryst. A. 2008, 64, 112-122.

S10 Sheldrick, G. M. Crystal Structure Refinement with SHELXL. Acta Cryst. A 2015, 71, 3-8.

S11 Dolomanov, O. V.; Bourhis, L. J.; Gildea, R. J.; Howard, J. A. K.; Puschmann, H. OLEX2: a Complete Structure Solution, Refinement and Analysis Program. J. Appl. Cryst. 2009, 42, 339341.

S12 Farrugia, L. J., WinGX and ORTEP for Windows: an update. J. Appl. Crystallogr. 2012, 45, 849-854. 
Table S1. Elemental analyses for the ligands $\mathbf{L 3}-\mathbf{L} 7$ and their complexes $\left[\mathbf{L} \boldsymbol{k}\left(\operatorname{Ln}(\mathrm{hfa})_{3}\right)_{m}\right]$. $(\mathrm{Ln}=\mathrm{La}, \mathrm{Eu}, \mathrm{Lu} ; k=3-6 ; m=1-2)$.

\begin{tabular}{|c|c|c|c|c|c|c|c|}
\hline Compound & $\begin{array}{c}\mathrm{MM} / \\
\mathrm{g} \cdot \mathrm{mol}^{-1}\end{array}$ & $\begin{array}{l}\% \mathrm{C} \\
\text { found }\end{array}$ & $\begin{array}{l}\% \mathrm{H} \\
\text { found }\end{array}$ & $\begin{array}{l}\% \mathrm{~N} \\
\text { found }\end{array}$ & $\begin{array}{l}\% \mathrm{C} \\
\text { calc }\end{array}$ & $\begin{array}{l}\% \mathrm{H} \\
\text { calc }\end{array}$ & $\begin{array}{l}\% \mathrm{~N} \\
\text { calc }\end{array}$ \\
\hline $\mathbf{L 3}\left(\mathrm{C}_{35} \mathrm{H}_{45} \mathrm{~N}_{5} \mathrm{O}\right)$ & 551.78 & 76.28 & 8.86 & 12.93 & 76.19 & 8.22 & 12.69 \\
\hline $\mathbf{L} 4\left(\mathrm{C}_{35} \mathrm{H}_{44} \mathrm{BrN}_{5} \mathrm{O}\right)$ & 630.68 & 66.45 & 7.50 & 11.07 & 66.66 & 7.03 & 11.10 \\
\hline $\mathbf{L 5}\left(\mathrm{C}_{35} \mathrm{H}_{43} \mathrm{Br}_{2} \mathrm{~N}_{5} \mathrm{O}\right)$ & 709.57 & 59.39 & 5.89 & 9.81 & 59.247 & 6.11 & 9.87 \\
\hline $\mathbf{L 6}\left(\mathrm{C}_{70} \mathrm{H}_{88} \mathrm{~N}_{10} \mathrm{O}_{2}\right)$ & 1101.54 & 76.18 & 8.86 & 12.94 & 76.33 & 8.05 & 12.72 \\
\hline $\mathbf{L} 7\left(\mathrm{C}_{105} \mathrm{H}_{131} \mathrm{~N}_{15} \mathrm{O}_{3}\right)$ & 1651.31 & 75.72 & 7.83 & 12.17 & 76.37 & 8.00 & 12.72 \\
\hline$\left[\mathrm{La}(\mathbf{L 3})(\mathrm{hfa})_{3}\right]$ & 1311.84 & 45.83 & 3.76 & 5.28 & 45.78 & 3.69 & 5.34 \\
\hline$\left[\mathrm{Eu}(\mathbf{L 3})(\mathrm{hfa})_{3}\right]$ & 1324.89 & 45.34 & 3.73 & 5.18 & 45.33 & 3.65 & 5.29 \\
\hline$\left[\mathrm{Lu}(\mathbf{L} \mathbf{3})(\mathrm{hfa})_{3}\right] \cdot 2 \mathrm{H}_{2} \mathrm{O}$ & 1347.9 & 43.77 & 3.71 & 4.29 & 43.39 & 3.79 & 5.06 \\
\hline$\left[\mathrm{Eu}(\mathbf{L 4})(\mathrm{hfa})_{3}\right]$ & 1403.79 & 42.87 & 3.49 & 4.93 & 42.78 & 3.37 & 4.99 \\
\hline$\left[\mathrm{Eu}(\mathbf{L 5})(\mathrm{hfa})_{3}\right]$ & 1482.70 & 40.51 & 3.13 & 4.57 & 40.50 & 3.13 & 4.72 \\
\hline $\begin{array}{l}{\left[\mathbf{L 6}\left(\mathrm{La}(\mathrm{hfa})_{3}\right)_{2}\right]} \\
\mathrm{C}_{100} \mathrm{H}_{94} \mathrm{~F}_{36} \mathrm{La}_{2} \mathrm{~N}_{10} \mathrm{O}_{14}\end{array}$ & 2621.66 & 45.60 & 3.57 & 5.15 & 45.81 & 3.61 & 5.34 \\
\hline $\begin{array}{l}{\left[\mathbf{L 6} 6\left(\mathrm{Eu}(\mathrm{hfa})_{3}\right)_{2}\right] \cdot 0.9} \\
\mathrm{C}_{6} \mathrm{H}_{6}\end{array}$ & 2718.08 & 47.08 & 3.92 & 5.67 & 46.58 & 3.69 & 5.15 \\
\hline $\begin{array}{l}\mathrm{C}_{100} \mathrm{H}_{94} \mathrm{~F}_{36} \mathrm{Eu}_{2} \mathrm{~N}_{10} \mathrm{O}_{14} . \\
0.9 \mathrm{C}_{6} \mathrm{H}_{6}\end{array}$ & & & & & & & \\
\hline
\end{tabular}



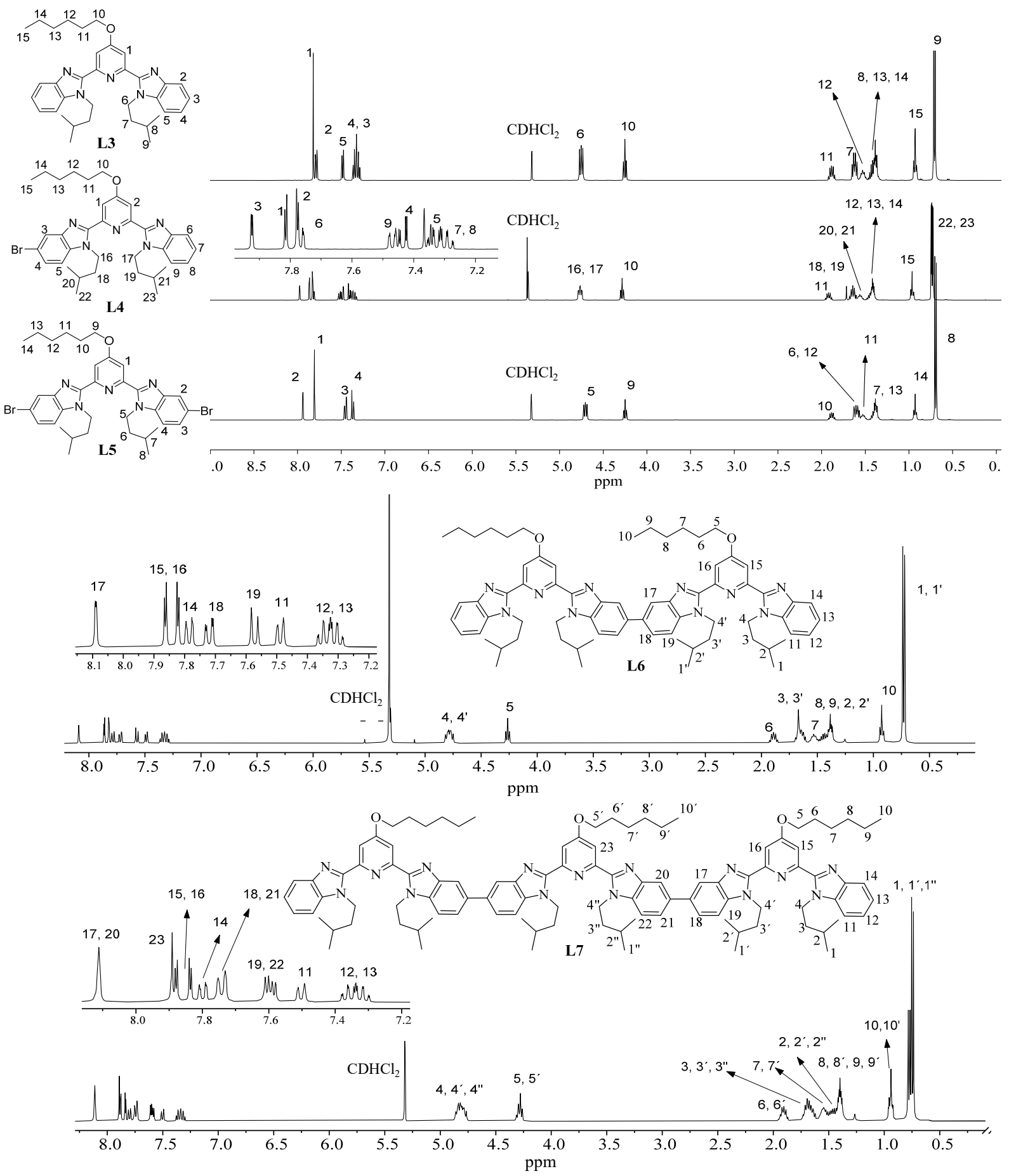

Figure S1 ${ }^{1} \mathrm{H}$ NMR spectra of ligands L3-L7 recorded in $\mathrm{CD}_{2} \mathrm{Cl}_{2}$ at $298 \mathrm{~K}$ 

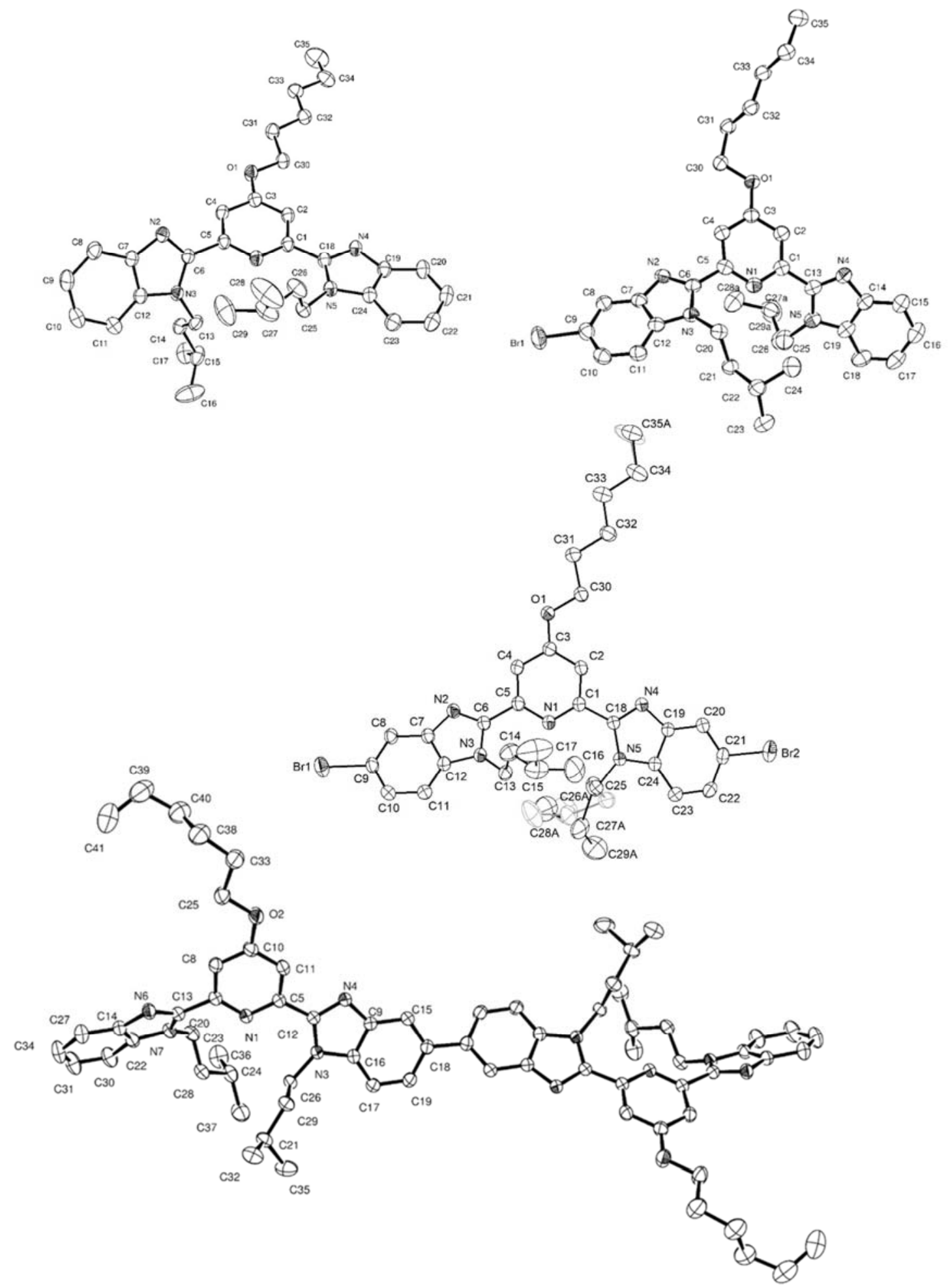

Figure S2 ORTEP views of the molecular structures of the ligands L3-L6 (Thermal ellipsoids drawn at $50 \%$ probability level) with numbering scheme. 
Table S2. Summary of crystal data, intensity measurements and structure refinements for L3-L5.

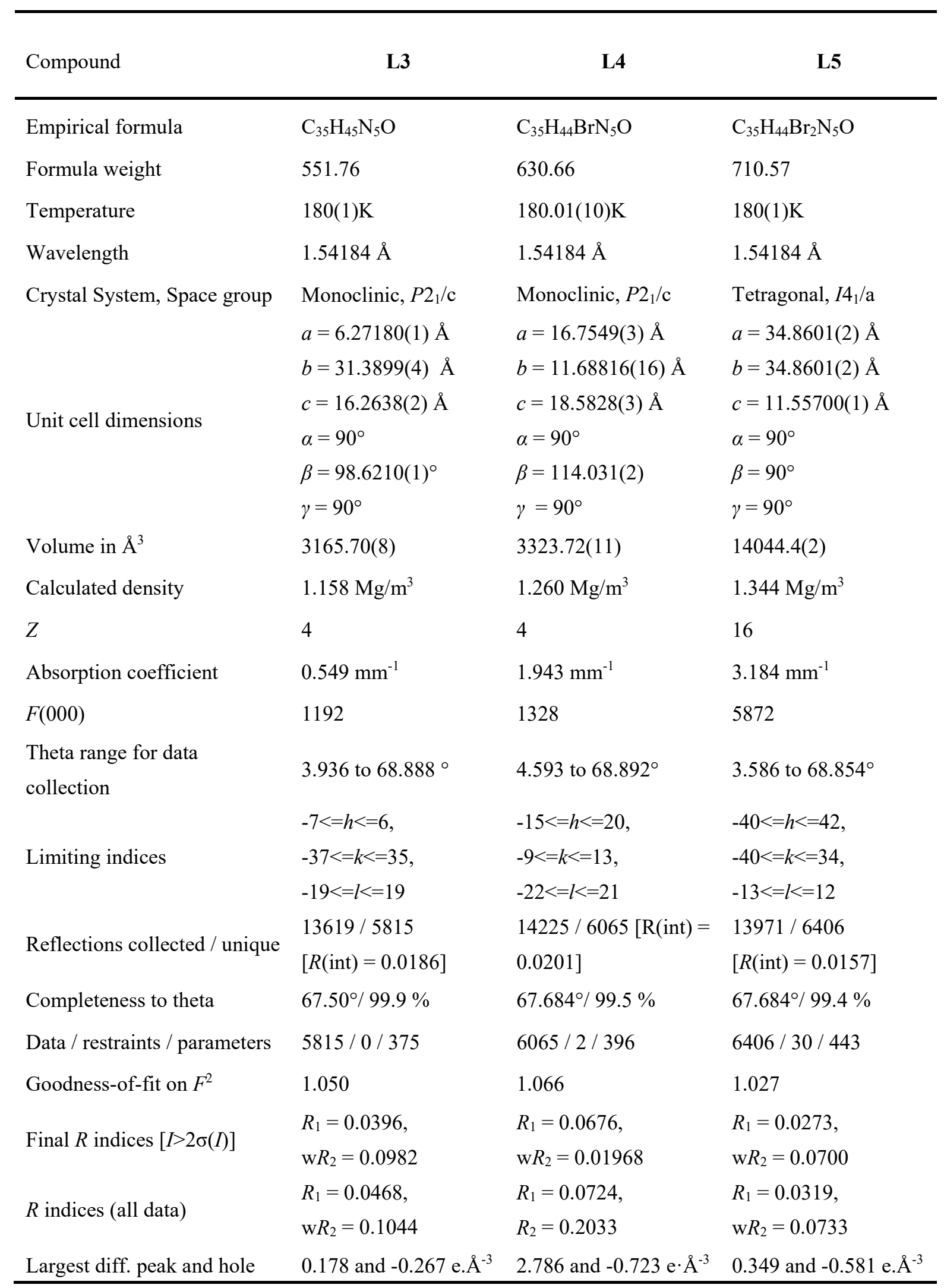


Table S3. Selected least-squares planes data of ligand L3.

\begin{tabular}{lccc}
\hline & Abbreviation & $\begin{array}{c}\text { Max. } \\
\text { deviation }(\AA)\end{array}$ & Atom \\
\hline $\begin{array}{l}\text { Pyridine } \\
\text { N(1) C (1) C(2) C(3) C(4) C(5) }\end{array}$ & Py & 0.012 & $\mathrm{C}(1,4)$ \\
$\begin{array}{l}\text { Benzimidazole 1 } \\
\text { C(6) N(2) C(7) C(8) C(9) C(10) C(11) C(12) N(3) }\end{array}$ & Bz1 & 0.044 & $\mathrm{C}(6)$ \\
$\begin{array}{l}\text { Benzimidazole 2 } \\
\text { C(18) N(4) C(19) C(20) C(21) C(22) C(23) C(24) N(5) }\end{array}$ & Bz2 & 0.022 & $\mathrm{C}(24)$ \\
\hline
\end{tabular}

Table S3. Selected least-squares planes data of ligand L4.

\begin{tabular}{lccc}
\hline & Abbreviation & $\begin{array}{c}\text { Max. } \\
\text { deviation/Å }\end{array}$ & Atom \\
\hline $\begin{array}{l}\text { Pyridine } \\
\text { N(1) C(1) C(2) C(3) C(4) C(5) }\end{array}$ & Py & 0.021 & $\mathrm{C}(1)$ \\
Benzimidazole 1 & Bz1 & 0.024 & $\mathrm{C}(9,11)$ \\
$\begin{array}{l}\text { C(13) N(4) C(14) C(15) C(16) C(17) C(18) C(19) N(5) } \\
\text { Benzimidazole 2 }\end{array}$ & Bz2 & 0.025 & $\mathrm{~N}(4)$ \\
$\mathrm{C}(6) \mathrm{N}(2) \mathrm{C}(7) \mathrm{C}(8) \mathrm{C}(9) \mathrm{C}(10) \mathrm{C}(11) \mathrm{C}(12) \mathrm{N}(3)$ & & & \\
\hline
\end{tabular}

Table S4. Selected least-squares planes data of ligand L5.

\begin{tabular}{lccc}
\hline & Abbreviation & $\begin{array}{c}\text { Max. } \\
\text { deviation/Å }\end{array}$ & Atom \\
\hline $\begin{array}{l}\text { Pyridine } \\
\text { N(1) C(1) C(2) C(3) C(4) C(5) }\end{array}$ & Py & 0.006 & $\mathrm{C}(1)$ \\
$\begin{array}{l}\text { Benzimidazole 1 } \\
\text { C(6) N(2) C(7) C(8) C(9) C(10) C(11) C(12) N(3) }\end{array}$ & Bz1 & 0.010 & $\mathrm{C}(7)$ \\
$\begin{array}{l}\text { Benzimidazole 2 } \\
\text { C(18) N(4) C(19) C(20) C(21) C(22) C(23) C(24) N(5) }\end{array}$ & Bz2 & 0.025 & $\mathrm{C}(19)$ \\
\hline
\end{tabular}

Table S5. Interplanar angles $\left(^{\circ}\right)$ of ligand L3-L5.

\begin{tabular}{|l|c|c|c|c|c|c|}
\hline \multirow{2}{*}{ Plane } & \multicolumn{3}{|c|}{ Benzimidazole (1) } & \multicolumn{3}{c|}{ Benzimidazole (2) } \\
\hline Ligand & L5 & L4 & L3 & L5 & L4 & L3 \\
\hline Pyridine & $20.53(6)^{\circ}$ & $36.20(1)^{\circ}$ & $55.42(4)^{\circ}$ & $26.10(6)^{\circ}$ & $34.07(1)^{\circ}$ & $3.39(4)^{\circ}$ \\
\hline Benzimidazole (1) & & & & $36.13(5)^{\circ}$ & $60.46(1)^{\circ}$ & $58.36(3)^{\circ}$ \\
\hline
\end{tabular}


Table S6 Summary of crystal data, intensity measurements and structure refinements for ligand L6.

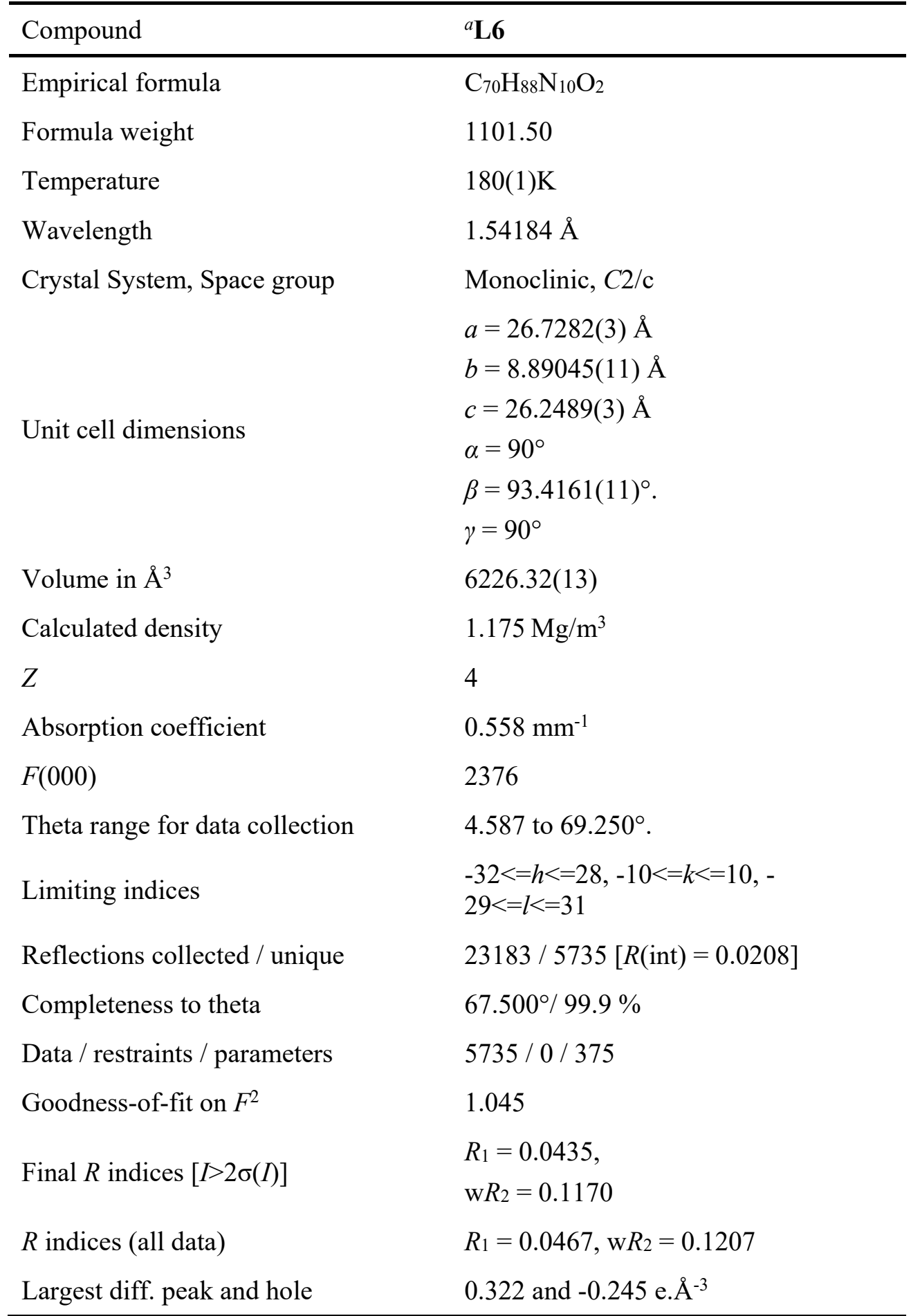

${ }^{a} \mathbf{L} 4$ crystallize in monoclinic space group (C2/c) and is located on a two-fold axis passing through the C18-C18' bond, so only half of the molecule is the asymmetric unit. 
Table S7. Selected least-squares planes data of ligand L6.

\begin{tabular}{|l|c|c|c|}
\hline & Abbreviation & $\begin{array}{c}\text { Max. } \\
\text { deviation/Å }\end{array}$ & Atom \\
\hline $\begin{array}{l}\text { Benzimidazole 2 } \\
\mathrm{C}(12) \mathrm{C}(15) \mathrm{N}(3) \mathrm{C}(17) \mathrm{C}(18) \mathrm{C}(16) \mathrm{C}(9) \mathrm{C}(19) \mathrm{N}(4)\end{array}$ & $\mathrm{Bz} 1$ & 0.021 & $\mathrm{C}(15)$ \\
\hline $\begin{array}{l}\text { Pyridine 1 } \\
\mathrm{N}(1) \mathrm{C}(5) \mathrm{C}(8) \mathrm{C}(10) \mathrm{C}(11) \mathrm{C}(13)\end{array}$ & Py1 & 0.004 & $\mathrm{C}(10,8)$ \\
\hline $\begin{array}{l}\text { Benzimidazole 1 } \\
\mathrm{C}(20) \mathrm{N}(6) \mathrm{C}(27) \mathrm{C}(30) \mathrm{C}(34) \mathrm{C}(22) \mathrm{C}(14) \mathrm{C}(31) \mathrm{N}(7)\end{array}$ & $\mathrm{Bz} 2$ & 0.012 & $\mathrm{C}(20)$ \\
\hline
\end{tabular}

Table S8. Interplanar angles $\left(^{\circ}\right)$ of ligand $\mathbf{L 6}$.

\begin{tabular}{|c|c|c|}
\hline Plane & Pyl $^{\mathrm{b}}$ & $\mathrm{Bz} 2$ \\
\hline $\mathrm{Bz}^{\mathrm{a}}$ & $15.13(4)$ & $46.81(4)$ \\
\hline Py1 & & $35.77(4)$ \\
\hline
\end{tabular}

${ }^{\mathrm{a} B e n z i m i d a z o l e ~ 1, ~}{ }^{\mathrm{b}}$ Pyridine 1 


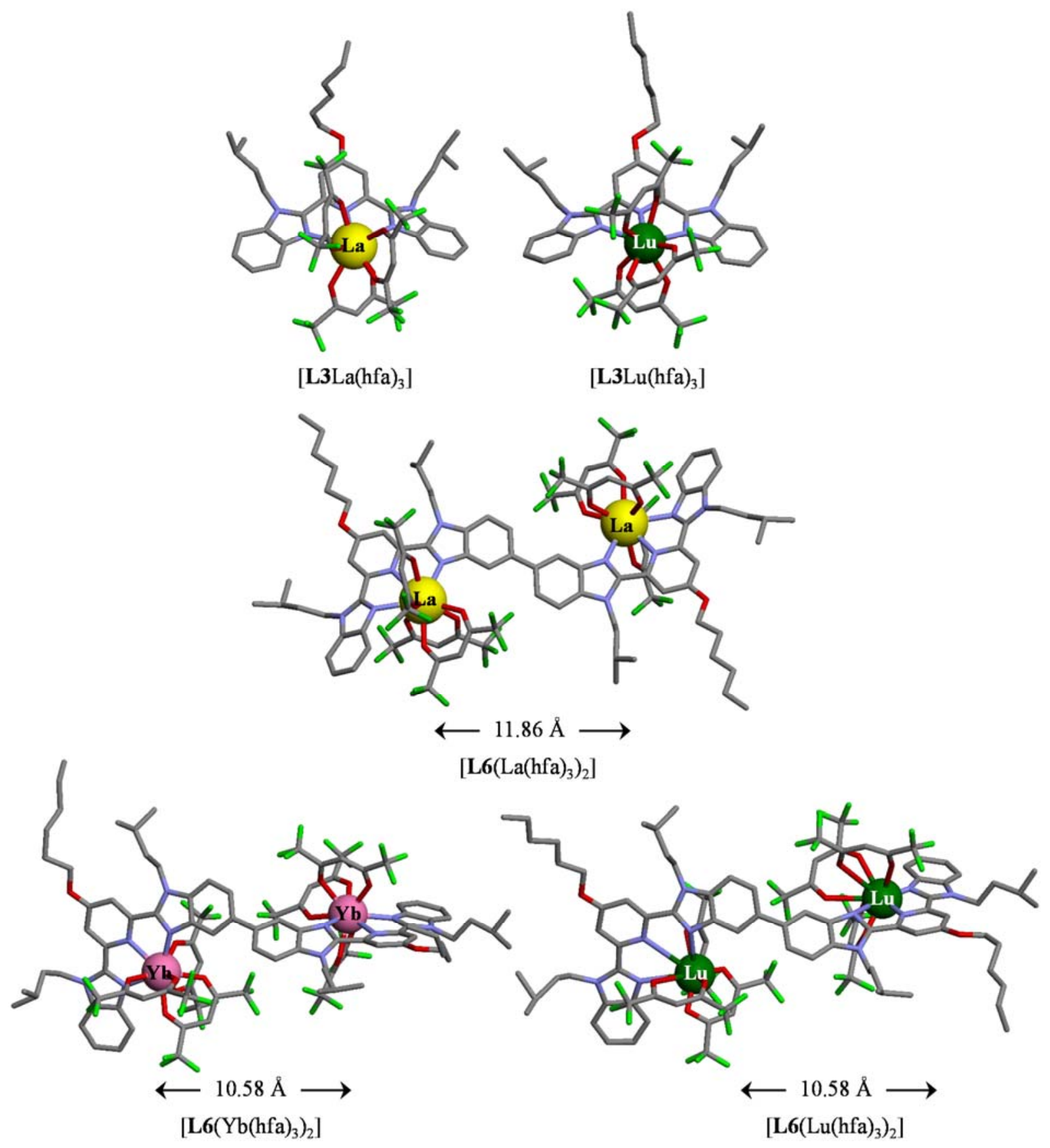

Figure S3. Views of the molecular structures of the complexes $\left[\mathbf{L 3} \operatorname{Ln}(\mathrm{hfa})_{3}\right](\mathrm{Ln}=\mathrm{La}, \mathrm{Lu})$ and $\left[\mathbf{L 6}\left(\operatorname{Ln}(\mathrm{hfa})_{3}\right)_{2}\right](\mathrm{Ln}=\mathrm{La}, \mathrm{Yb}, \mathrm{Lu})$ as found in their crystal structures. Hydrogen atoms are omitted for clarity. Color code: grey $=$ carbon, blue $=$ nitrogen, red $=$ oxygen, light green $=$ fluorine, yellow $=$ lanthanum, purple $=$ ytterbium, dark green $=$ lutetium . 
Table S9. Summary of crystal data, intensity measurements and structure refinements of complexes $\left[\mathbf{L k} \operatorname{Ln}(\mathrm{hfa})_{3}\right],(\mathrm{Ln}=\mathrm{La}, \mathrm{Eu}, \mathrm{Lu}$ and $\boldsymbol{k}=3-5)$.

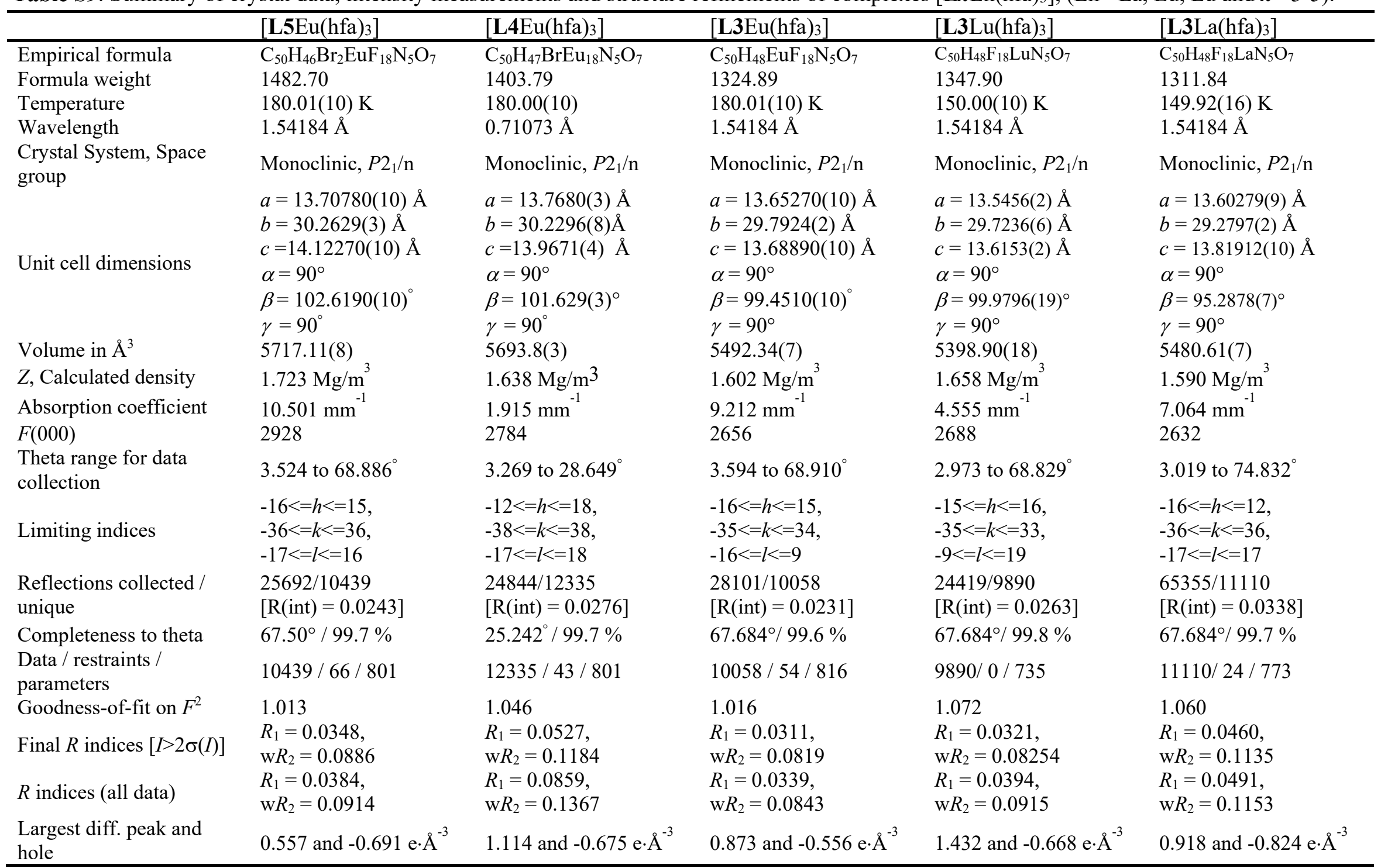


Table S10 Summary of crystal data, intensity measurements and structure refinements complexes $\left[\mathbf{L 6}\left(\operatorname{Ln}(\mathrm{hfa})_{3}\right)_{2}\right],(\mathrm{Ln}=\mathrm{Eu}, \mathrm{La}, \mathrm{Yb}, \mathrm{Lu})$.

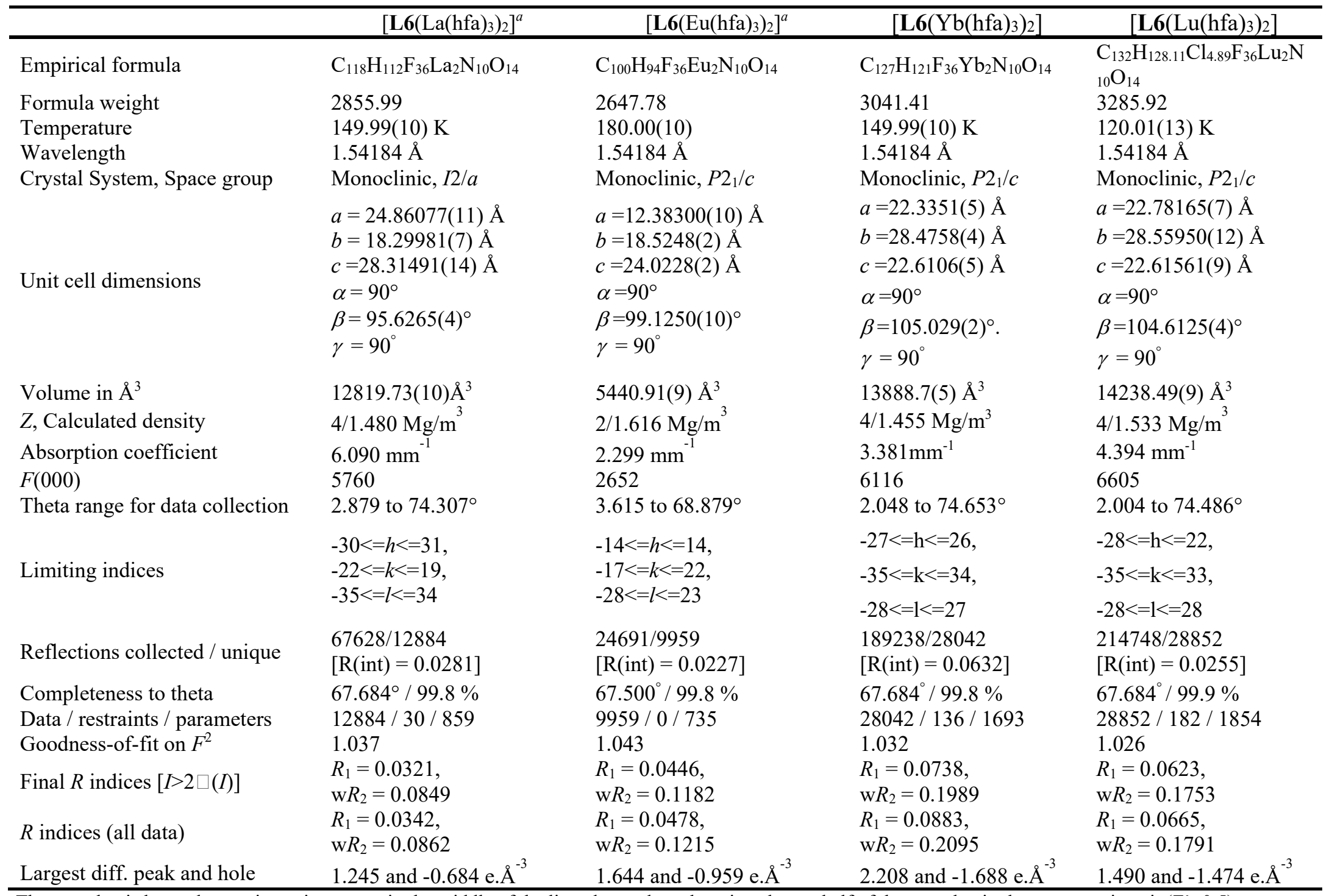

${ }^{a}$ The complex is located on an inversion center in the middle of the ligand strand, so there is only one half of the complex in the asymmetric unit (Z' $\left.=0.5\right)$ 


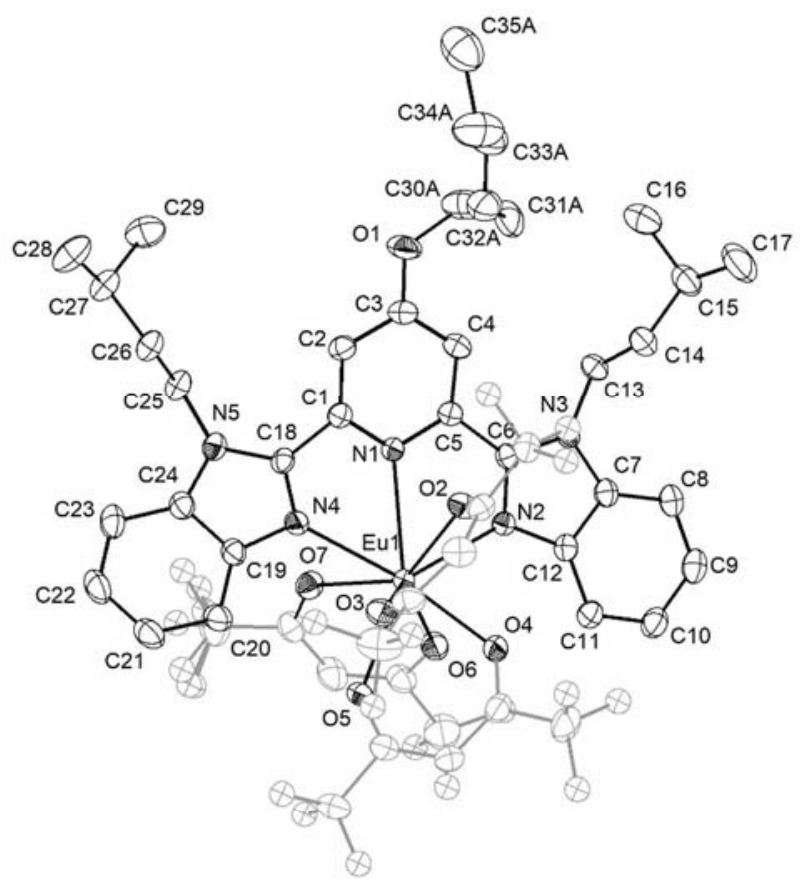

$\left[\mathrm{L3Eu}(\mathrm{hfa})_{3}\right]$

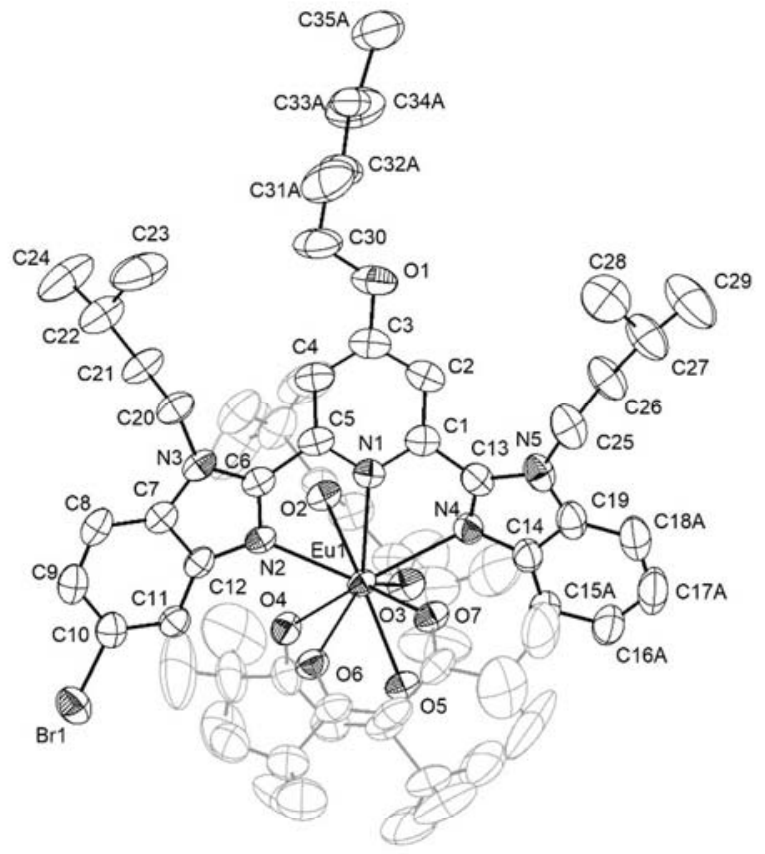

$\left[\mathrm{L} 4 \mathrm{Eu}(\mathrm{hfa})_{3}\right]$

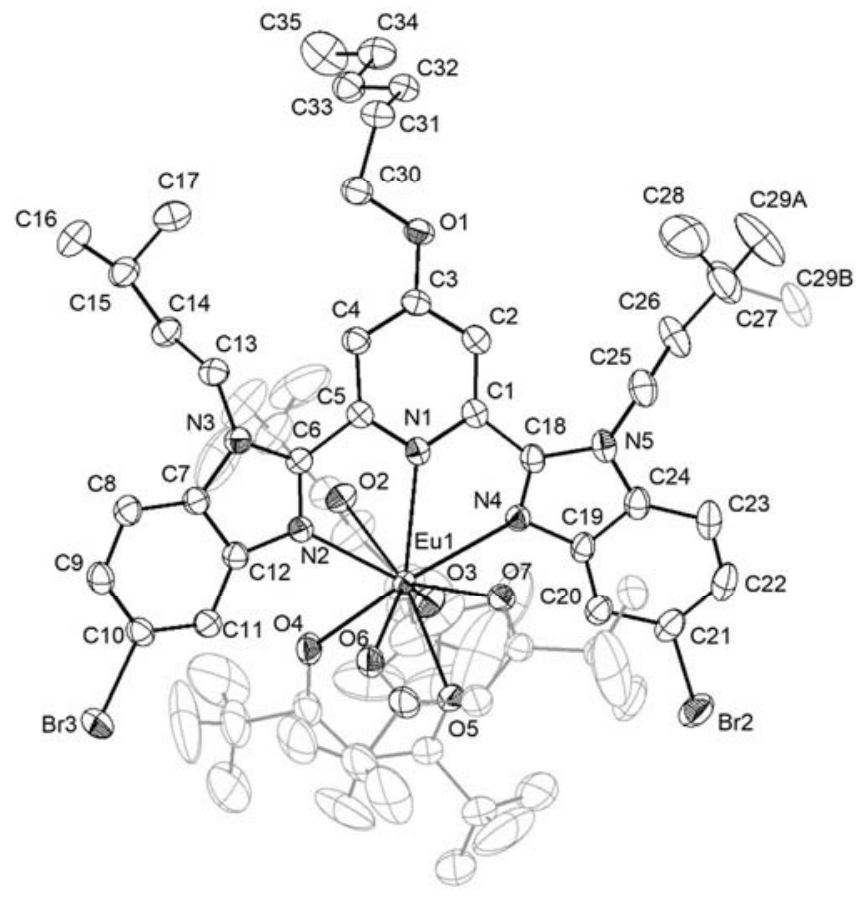

\section{$\left[\mathrm{L5Eu}(\mathrm{hfa})_{3}\right]$}

Figure S4. ORTEP views for complexes $\left[\mathbf{L k E u}(\mathrm{hfa})_{3}\right](\boldsymbol{k}=3-5)$ with atomic numbering scheme. Ellispoïds are shown at $40 \%$ probability level. 


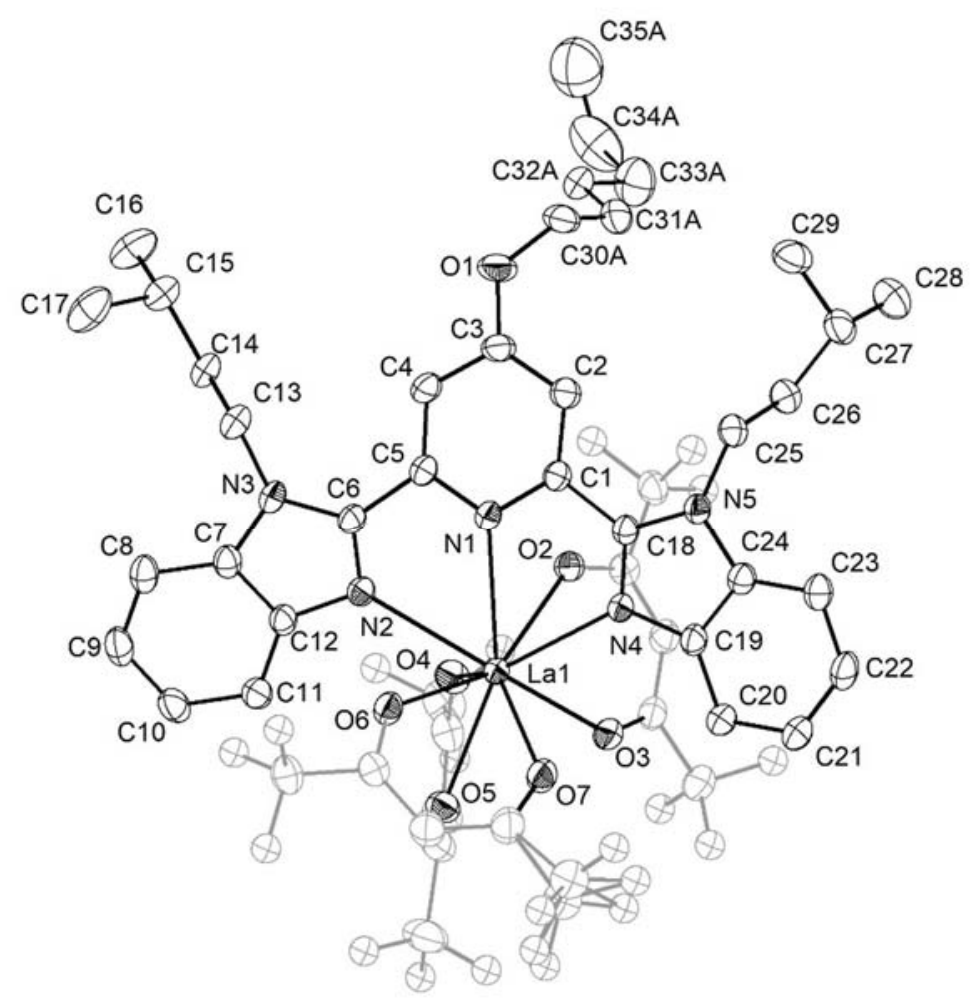

$\left[\mathbf{L} 3 \mathrm{La}(\mathrm{hfa})_{3}\right]$

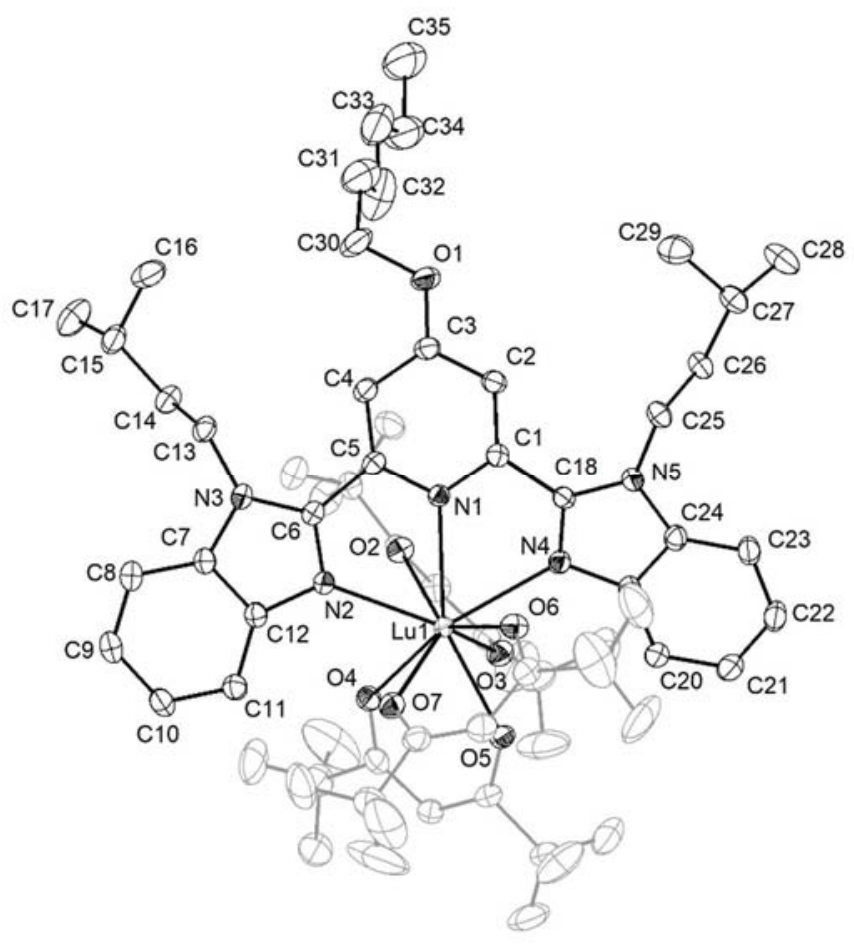

$\left[\mathbf{L 3} \mathrm{Lu}(\mathrm{hfa})_{3}\right]$

Figure S5. ORTEP views for complexes $\left[\mathbf{L 3} \operatorname{Ln}(\mathrm{hfa})_{3}\right](\mathrm{Ln}=\mathrm{La}, \mathrm{Lu})$ with atomic numbering scheme. Ellispoïds are shown at $40 \%$ probability level. 

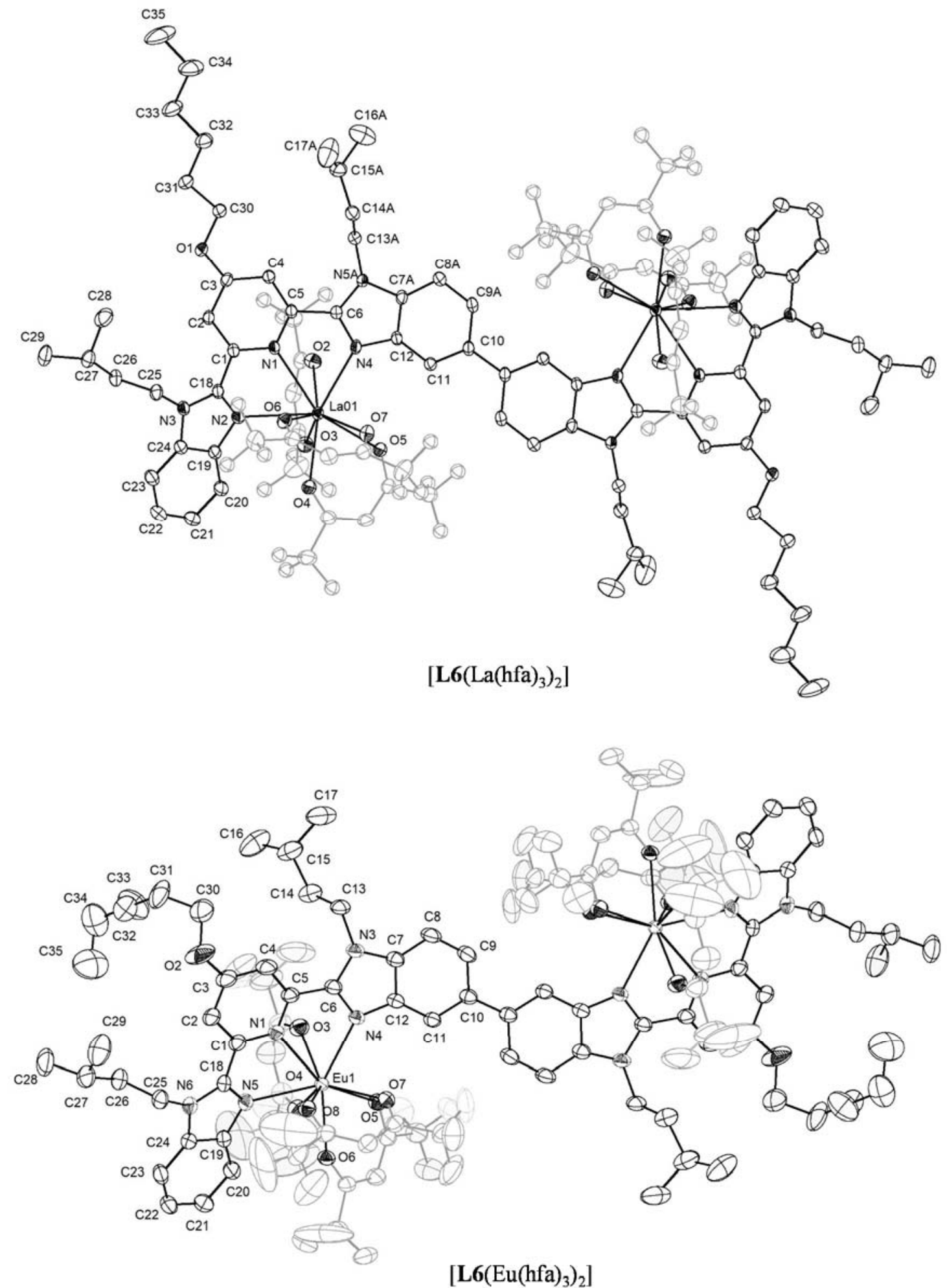

Figure S6. ORTEP views for complexes $\left[\mathbf{L 6}\left(\operatorname{Ln}(\mathrm{hfa})_{3}\right)_{2}\right](\mathrm{Ln}=\mathrm{La}, \mathrm{Eu})$ with atomic numbering scheme. Ellispoïds are shown at $40 \%$ probability level. 

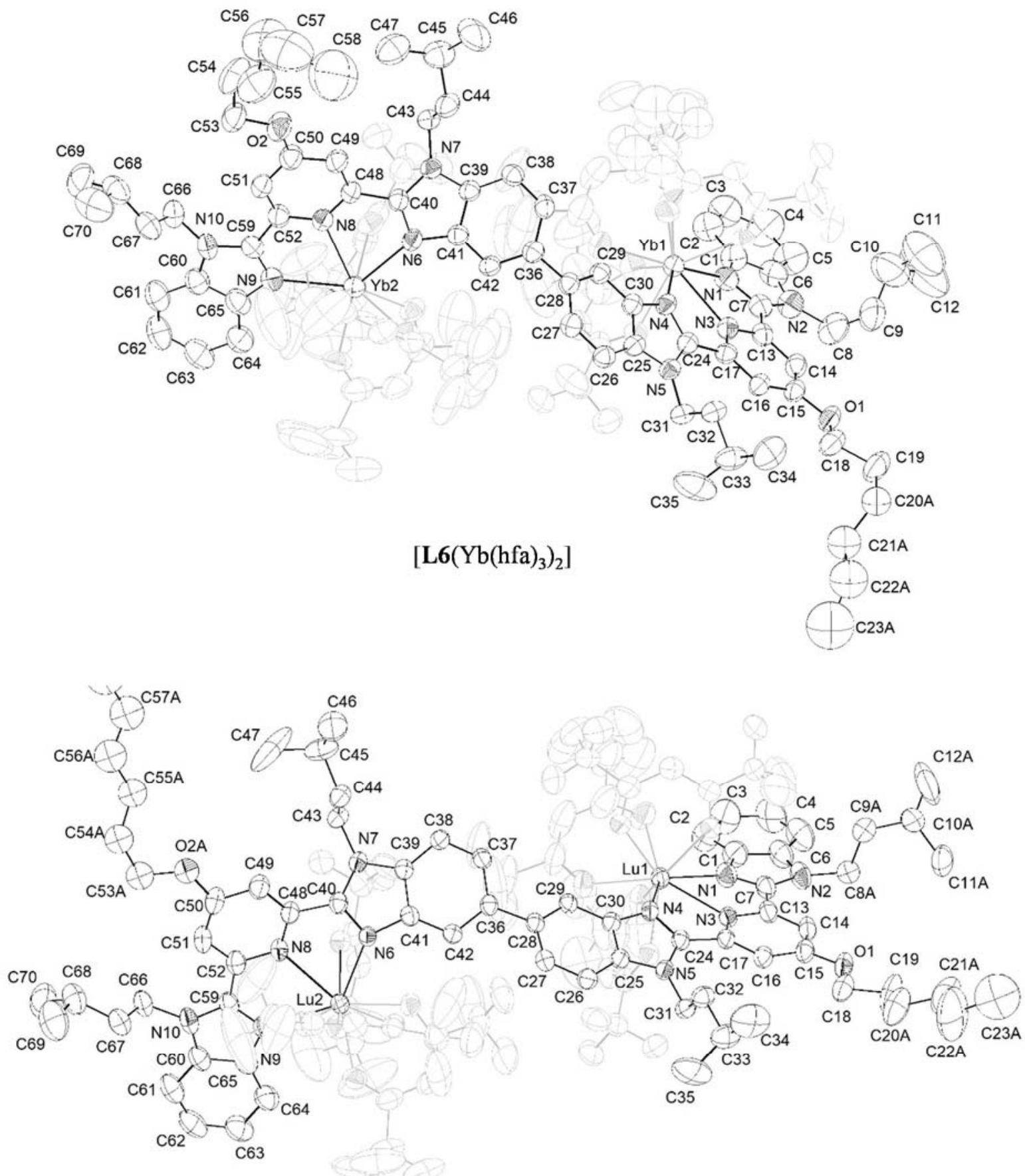

$\left[\mathrm{L6}\left(\mathrm{Lu}(\mathrm{hfa})_{3}\right)_{2}\right]$

Figure S7. ORTEP views for complexes $\left[\mathbf{L 6}\left(\operatorname{Ln}(\mathrm{hfa})_{3}\right)_{2}\right](\mathrm{Ln}=\mathrm{Yb}, \mathrm{Lu})$ with atomic numbering scheme. Ellispoïds are shown at $40 \%$ probability level. 
Table S11. Deviations from ideal nine-coordinated geometries computed with program Shape 2.1 for complexes $\left[\mathbf{L} k \operatorname{Ln}(\mathrm{hfa})_{3}\right]$, $(\boldsymbol{k}=3-5$ and $\mathrm{Ln}=\mathrm{La}, \mathrm{Eu}, \mathrm{Lu})$.

\begin{tabular}{|c|c|c|c|c|c|c|}
\hline Shape & $\begin{array}{l}\text { Point } \\
\text { group }\end{array}$ & {$\left[\mathbf{L 5} \mathrm{Eu}(\mathrm{hfa})_{3}\right]$} & {$\left[\mathbf{L} 4 \mathrm{Eu}(\mathrm{hfa})_{3}\right]$} & {$\left[\mathbf{L} 3 \mathrm{Eu}(\mathrm{hfa})_{3}\right]$} & {$\left[\mathbf{L 3 L a}(\mathrm{hfa})_{3}\right]$} & {$\left[\mathbf{L 3} \mathrm{Lu}(\mathrm{hfa})_{3}\right]$} \\
\hline Enneagon & $D_{9 \mathrm{~h}}$ & 35.989 & 35.625 & 35.820 & 34.964 & 36.573 \\
\hline Octagonal pyramid & $C_{8 \mathrm{v}}$ & 21.239 & 21.152 & 21.119 & 21.358 & 21.129 \\
\hline Heptagonal bipyramid & $D_{7 \mathrm{~h}}$ & 16.943 & 16.862 & 17.708 & 16.870 & 18.380 \\
\hline Triangular cupola $(\mathrm{J} 3)$ & $C_{3 \mathrm{v}}$ & 15.440 & 15.064 & 15.585 & 14.618 & 16.449 \\
\hline Capped cube J8 & $C_{4 \mathrm{v}}$ & 9.371 & 9.158 & 9.443 & 7.930 & 9.761 \\
\hline Spherical-relaxed capped cube & $C_{4 \mathrm{v}}$ & 7.925 & 7.734 & 8.073 & 6.333 & 8.491 \\
\hline Capped square antiprism J10 & $C_{4 \mathrm{v}}$ & 2.186 & 2.270 & 2.081 & 3.318 & 1.660 \\
\hline Spherical capped square antiprism & $C_{4 \mathrm{v}}$ & 1.216 & 1.358 & 1.194 & 2.354 & 0.854 \\
\hline Tricapped trigonal prism J51 & $D_{3 \mathrm{~h}}$ & 3.602 & 3.721 & 3.525 & 4.579 & 2.915 \\
\hline Spherical tricapped trigonal prism & $D_{3 \mathrm{~h}}$ & 2.247 & 2.321 & 2.109 & 3.005 & 1.733 \\
\hline Tridiminished icosahedron J63 & $C_{3 \mathrm{v}}$ & 12.184 & 11.887 & 11.695 & 11.887 & 12.418 \\
\hline Hula-hoop & $C_{2 \mathrm{v}}$ & 9.669 & 9.520 & 9.842 & 9.286 & 10.063 \\
\hline Muffin & $C_{\mathrm{s}}$ & 0.876 & 0.972 & 0.886 & 1.495 & 0.782 \\
\hline
\end{tabular}


Table S12. Selected bond distances $(\AA)$ in $\left[\mathbf{L k} \operatorname{Ln}(\mathrm{hfa})_{3}\right],(\boldsymbol{k}=3-5$ and $\mathrm{Ln}=\mathrm{La}, \mathrm{Eu}, \mathrm{Lu})$.

\begin{tabular}{|c|c|c|c|c|c|}
\hline \multirow[b]{2}{*}{ Atoms $1-2$} & \multicolumn{5}{|c|}{ Selected Bond Distances $(\AA)$} \\
\hline & {$\left[\mathbf{L 5 E u}(\mathrm{hfa})_{3}\right]$} & {$\left[\mathrm{L4Eu}(\mathrm{hfa})_{3}\right]$} & {$\left[\mathbf{L 3 E u}(\mathrm{hfa})_{3}\right]$} & {$\left[\mathbf{L 3} \mathrm{Lu}(\mathrm{hfa})_{3}\right]$} & {$\left[\mathbf{L 3 L a}(\mathrm{hfa})_{3}\right]$} \\
\hline $\mathrm{Ln}-\mathrm{N}(1)$ & $2.628(3)$ & $2.647(4)$ & $2.626(2)$ & $2.532(3)$ & $2.767(3)$ \\
\hline $\mathrm{Ln}-\mathrm{N}(2)$ & $2.560(3)$ & $2.561(4)$ & $2.529(2)$ & $2.456(3)$ & $2.618(3)$ \\
\hline $\mathrm{Ln}-\mathrm{N}(4)$ & $2.563(3)$ & $2.540(4)$ & $2.529(2)$ & $2.468(3)$ & $2.666(3)$ \\
\hline $\mathrm{Ln}-\mathrm{O}(2)$ & $2.420(2)$ & $2.419(3)$ & $2.421(2)$ & $2.323(2)$ & $2.572(3)$ \\
\hline $\mathrm{Ln}-\mathrm{O}(3)$ & $2.450(3)$ & $2.459(4)$ & $2.471(2)$ & $2.433(2)$ & $2.495(3)$ \\
\hline $\mathrm{Ln}-\mathrm{O}(4)$ & $2.377(2)$ & $2.377(3)$ & $2.375(2)$ & $2.283(2)$ & $2.532(3)$ \\
\hline $\mathrm{Ln}-\mathrm{O}(5)$ & $2.402(2)$ & $2.414(3)$ & $2.400(2)$ & $2.313(2)$ & $2.502(3)$ \\
\hline $\mathrm{Ln}-\mathrm{O}(6)$ & $2.421(2)$ & $2.433(4)$ & $2.443(2)$ & $2.336(2)$ & $2.526(3)$ \\
\hline $\mathrm{Ln}-\mathrm{O}(7)$ & $2.427(2)$ & $2.435(4)$ & $2.424(2)$ & $2.390(2)$ & $2.510(3)$ \\
\hline
\end{tabular}

Table S13. Chelate angles $\left(^{\circ}\right)$ in $\left[\mathbf{L} \boldsymbol{k L n}(\mathrm{hfa})_{3}\right],(\boldsymbol{k}=3-5$ and $\mathrm{Ln}=\mathrm{La}, \mathrm{Eu}, \mathrm{Lu})$.

\begin{tabular}{|c|c|c|c|c|c|}
\hline \multirow[b]{2}{*}{ Atoms 1-2-3 } & \multicolumn{5}{|c|}{ Chelate Angles $\left(^{\circ}\right)$} \\
\hline & {$\left[\mathbf{L 5 E u}(\mathrm{hfa})_{3}\right]$} & {$\left[\mathbf{L 4 E u}(\mathrm{hfa})_{3}\right]$} & {$\left[\mathbf{L 3 E u}(\mathrm{hfa})_{3}\right]$} & {$\left[\mathbf{L 3} \operatorname{Lu}(\mathrm{hfa})_{3}\right]$} & {$\left[\mathbf{L 3 L a}(\mathrm{hfa})_{3}\right]$} \\
\hline $\mathrm{N}(1)-\operatorname{Ln}-\mathrm{N}(2)$ & $62.63(8)$ & $62.50(13)$ & $63.02(6)$ & $64.91(8)$ & $61.35(10)$ \\
\hline $\mathrm{N}(1)-\operatorname{Ln}-\mathrm{N}(4)$ & $63.63(8)$ & $63.63(14)$ & $64.01(6)$ & $65.93(8)$ & $59.91(10)$ \\
\hline $\mathrm{N}(2)-\operatorname{Ln}-\mathrm{N}(4)$ & $126.17(9)$ & $126.06(14)$ & $126.93(7)$ & $130.72(9)$ & $121.14(10)$ \\
\hline $\mathrm{O}(2)-\mathrm{Ln}-\mathrm{O}(3)$ & $69.12(8)$ & $68.89(12)$ & $68.28(6)$ & $69.57(8)$ & $68.71(9)$ \\
\hline $\mathrm{O}(4)-\mathrm{Ln}-\mathrm{O}(5)$ & $73.75(8)$ & $73.50(12)$ & $73.79(7)$ & $76.54(8)$ & $67.23(10)$ \\
\hline $\mathrm{O}(6)-\mathrm{Ln}-\mathrm{O}(7)$ & $69.37(8)$ & $69.18(13)$ & $68.95(7)$ & $70.20(8)$ & $67.09(9)$ \\
\hline
\end{tabular}


Table S14. Selected bond angles $\left(^{\circ}\right)$ in $\left[\mathbf{L} \boldsymbol{k L n}(\mathrm{hfa})_{3}\right],(\boldsymbol{k}=3-5$ and $\mathrm{Ln}=\mathrm{La}, \mathrm{Eu}, \mathrm{Lu})$

\begin{tabular}{|c|c|c|c|c|c|}
\hline \multirow[b]{2}{*}{ Atoms 1-2-3 } & \multicolumn{5}{|c|}{ Angles $\left({ }^{\circ}\right)$} \\
\hline & {$\left[\mathbf{L 5 E u}(\mathrm{hfa})_{3}\right]$} & {$\left[\mathrm{L} 4 \mathrm{Eu}(\mathrm{hfa})_{3}\right]$} & {$\left[\mathbf{L 3 E u}(\mathrm{hfa})_{3}\right]$} & {$\left[\mathbf{L 3 L u}(\mathrm{hfa})_{3}\right]$} & [L3La(hfa) $\left.{ }_{3}\right]$ \\
\hline $\mathrm{O}(2)-\mathrm{Ln}-\mathrm{N}(1)$ & $66.62(8)$ & $66.41(12)$ & $64.79(6)$ & $65.51(8)$ & $65.09(10)$ \\
\hline $\mathrm{O}(2)-\mathrm{Ln}-\mathrm{N}(2)$ & $69.95(9)$ & $70.38(12)$ & $70.36(6)$ & $71.30(8)$ & $89.72(10)$ \\
\hline $\mathrm{O}(2)-\mathrm{Ln}-\mathrm{N}(4)$ & $86.60(9)$ & $86.25(13)$ & $84.89(7)$ & $85.78(8)$ & $69.28(10)$ \\
\hline $\mathrm{O}(3)-\mathrm{Ln}-\mathrm{N}(1)$ & $115.42(9)$ & $115.41(13)$ & $114.87(6)$ & $116.46(8)$ & $127.29(9)$ \\
\hline $\mathrm{O}(3)-\mathrm{Ln}-\mathrm{N}(2)$ & $134.80(9)$ & $134.89(13)$ & $133.33(7)$ & $134.31(8)$ & $141.95(10)$ \\
\hline $\mathrm{O}(3)-\mathrm{Ln}-\mathrm{N}(4)$ & $69.21(10)$ & $69.36(15)$ & $69.40(7)$ & $68.28(8)$ & $81.22(10)$ \\
\hline $\mathrm{O}(4)-\mathrm{Ln}-\mathrm{N}(1)$ & $134.23(9)$ & $133.33(13)$ & $132.73(6)$ & $133.13(8)$ & $110.24(10)$ \\
\hline $\mathrm{O}(4)-\mathrm{Ln}-\mathrm{N}(2)$ & $80.47(9)$ & $80.11(13)$ & $79.43(7)$ & $77.92(8)$ & $70.37(10)$ \\
\hline $\mathrm{O}(4)-\mathrm{Ln}-\mathrm{N}(4)$ & $141.01(10)$ & $140.96(14)$ & $140.07(7)$ & 138.97(9) & $135.27(10)$ \\
\hline $\mathrm{O}(5)-\mathrm{Ln}-\mathrm{N}(1)$ & $152.01(8)$ & $153.15(12)$ & $153.44(6)$ & $150.31(8)$ & $156.27(9)$ \\
\hline $\mathrm{O}(5)-\mathrm{Ln}-\mathrm{N}(2)$ & $137.38(9)$ & $136.68(13)$ & $136.17(7)$ & $135.97(8)$ & $97.11(10)$ \\
\hline $\mathrm{O}(5)-\mathrm{Ln}-\mathrm{N}(4)$ & $94.27(9)$ & $95.18(14)$ & $95.07(7)$ & $91.08(8)$ & $139.33(10)$ \\
\hline $\mathrm{O}(6)-\mathrm{Ln}-\mathrm{N}(1)$ & $112.16(9)$ & $112.54(13)$ & $112.27(6)$ & $78.25(8)$ & $88.27(9)$ \\
\hline $\mathrm{O}(6)-\mathrm{Ln}-\mathrm{N}(2)$ & $70.38(9)$ & $70.32(13)$ & $70.59(7)$ & $106.02(8)$ & $66.07(10)$ \\
\hline $\mathrm{O}(6)-\mathrm{Ln}-\mathrm{N}(4)$ & $135.80(9)$ & $136.10(14)$ & $135.39(7)$ & $66.56(8)$ & 108.51(10) \\
\hline $\mathrm{O}(7)-\mathrm{Ln}-\mathrm{N}(1)$ & $80.66(8)$ & $82.70(12)$ & $81.50(7)$ & $112.98(8)$ & 115.99(10) \\
\hline $\mathrm{O}(7)-\mathrm{Ln}-\mathrm{N}(2)$ & $107.02(9)$ & $108.23(13)$ & $108.05(7)$ & $69.50(8)$ & $133.14(10)$ \\
\hline $\mathrm{O}(7)-\mathrm{Ln}-\mathrm{N}(4)$ & $66.55(9)$ & $66.96(14)$ & $66.55(7)$ & $135.96(8)$ & $73.26(10)$ \\
\hline
\end{tabular}


Table S15. Selected least-squares planes data of complex $\left[\mathbf{L 3 E u}\left((\mathrm{hfa})_{3}\right]\right.$.

\begin{tabular}{lccc}
\hline & Abbreviation & $\begin{array}{c}\text { Max. } \\
\text { deviation }(\AA)\end{array}$ & Atom \\
\hline $\begin{array}{l}\text { Pyridine } \\
\text { N(1) C(1) C(2) C(3) C(4) C(5) }\end{array}$ & Py & 0.014 & $\mathrm{C}(3)$ \\
$\begin{array}{l}\text { Benzimidazole 1 } \\
\text { C(6) N(2) C(7) C(8) C(9) C(10) C(11) C(12) N(3) }\end{array}$ & Bz 1 & 0.010 & $\mathrm{~N}(3), \mathrm{C}(7)$ \\
$\begin{array}{l}\text { Benzimidazole 2 } \\
\text { C(18) N(4) C(19) C(20) C(21) C(22) C(23) C(24) N(5) }\end{array}$ & Bz 2 & 0.023 & $\mathrm{C}(24)$ \\
$\begin{array}{l}\text { Hexafluoroacetylacetonate } \\
\text { C(36) C(37) C(38) O(2) O(3) }\end{array}$ & Hfa1 & 0.017 & $\mathrm{C}(38)$ \\
$\begin{array}{l}\text { Hexafluoroacetylacetonate } \\
\text { C(41) C(42) C(43) O(4) O(5) }\end{array}$ & Hfa2 & 0.021 & $\mathrm{C}(41)$ \\
$\begin{array}{l}\text { Hexafluoroacetylacetonate } \\
\text { C(46) C(47) C(48) O(6) O(7) }\end{array}$ & Hfa3 & 0.013 & $\mathrm{C}(46)$ \\
\hline
\end{tabular}

Table S16. Selected least-squares planes data of complex $\left[\mathbf{L} 4 \mathrm{Eu}\left((\mathrm{hfa})_{3}\right]\right.$..

\begin{tabular}{lccc}
\hline & Abbreviation & $\begin{array}{c}\text { Max. deviation } \\
(\AA)\end{array}$ & Atom \\
\hline $\begin{array}{l}\text { Pyridine } \\
\text { N(1) C(1) C(2) C(3) C(4) C(5) }\end{array}$ & Py & 0.008 & $\mathrm{C}(3)$ \\
$\begin{array}{l}\text { Benzimidazole 1 } \\
\text { C(6) N(2) C(7) C(8) C(9) C(10) C(11) C(12) N(3) }\end{array}$ & Bz 1 & 0.012 & $\mathrm{C}(6), \mathrm{N}(3)$ \\
$\begin{array}{l}\text { Benzimidazole 2 } \\
\text { C(13) N(4) C(14) C(15A) C(16A) C(17A) C(18A) }\end{array}$ & Bz 2 & 0.028 & $\begin{array}{c}\mathrm{C}(17 \mathrm{~A}), \mathrm{C}( \\
\mathrm{C}(19) \mathrm{N}(5)\end{array}$ \\
$\begin{array}{l}\text { Hexafluoroacetylacetonate } \\
\text { C(36) C(37) C(38) O(2) O(3) }\end{array}$ & Hfa1 & 0.02 & $\mathrm{C}(36)$ \\
$\begin{array}{l}\text { Hexafluoroacetylacetonate } \\
\text { C(41) C(42) C(43) O(4) O(5) }\end{array}$ & Hfa2 & 0.014 & $\mathrm{C}(43)$ \\
$\begin{array}{l}\text { Hexafluoroacetylacetonate } \\
\text { C(46) C(47) C(48) O(6) O(7) }\end{array}$ & Hfa3 & 0.009 & $\mathrm{C}(48)$ \\
\hline
\end{tabular}


Table S17. Selected least-squares planes data of complex [L5Eu((hfa) 3$]$..

\begin{tabular}{lccc}
\hline & Abbreviation & $\begin{array}{c}\text { Max. } \\
\text { deviation( }(\AA)\end{array}$ & Atom \\
\hline $\begin{array}{l}\text { Pyridine } \\
\text { N(1) C(1) C(2) C(3) C(4) C(5) }\end{array}$ & Py & 0.008 & $\mathrm{C}(5)$ \\
$\begin{array}{l}\text { Benzimidazole 1 } \\
\text { C(6) N(2) C(7) C(8) C(9) C(10) C(11) C(12) N(3) }\end{array}$ & Bz 1 & 0.013 & $\mathrm{C}(6)$ \\
Benzimidazole 2 & Bz 2 & 0.018 & $\mathrm{C}(24)$ \\
$\mathrm{C}(18)$ N(4) C(19) C(20) C(21) C(22) C(23) C(24) N(5) & & & \\
Hexafluoroacetylacetonate & Hfa1 & 0.015 & $\mathrm{C}(38)$ \\
C(36) C(37) C(38) O(2) O(3) & & & \\
$\begin{array}{l}\text { Hexafluoroacetylacetonate } \\
\text { C(41) C(42) C(43) O(4) O(5) }\end{array}$ & Hfa2 & 0.016 & $\mathrm{C}(43)$ \\
Hexafluoroacetylacetonate & & & \\
C(46) C(47) C(48) O(6) O(7) & Hfa3 & 0.010 & $\mathrm{C}(46)$ \\
\hline
\end{tabular}

Table S18. Selected least-squares planes data of complex [L3Lu((hfa) $\left.{ }_{3}\right]$.

\begin{tabular}{|c|c|c|c|}
\hline & Abbreviation & $\begin{array}{c}\text { Max. } \\
\text { deviation }(\AA)\end{array}$ & Atom \\
\hline $\begin{array}{l}\text { Pyridine } \\
N(1) C(1) C(2) C(3) C(4) C(5)\end{array}$ & Py & 0.016 & $\mathrm{C}(3)$ \\
\hline $\begin{array}{l}\text { Benzimidazole } 1 \\
C(6) N(2) C(7) C(8) C(9) C(10) C(11) C(12) N(3)\end{array}$ & Bzl & 0.016 & $C(12)$ \\
\hline $\begin{array}{l}\text { Benzimidazole } 2 \\
C(18) N(4) C(19) C(20) C(21) C(22) C(23) C(24) N(5)\end{array}$ & $\mathrm{Bz} 2$ & 0.024 & $C(24)$ \\
\hline $\begin{array}{l}\text { Hexafluoroacetylacetonate } \\
\mathrm{C}(36) \mathrm{C}(37) \mathrm{C}(38) \mathrm{O}(2) \mathrm{O}(3)\end{array}$ & Hfa1 & 0.023 & $\mathrm{C}(38)$ \\
\hline $\begin{array}{l}\text { Hexafluoroacetylacetonate } \\
\mathrm{C}(41) \mathrm{C}(42) \mathrm{C}(43) \mathrm{O}(4) \mathrm{O}(5)\end{array}$ & $\mathrm{Hfa} 2$ & 0.029 & $\mathrm{C}(41)$ \\
\hline $\begin{array}{l}\text { Hexafluoroacetylacetonate } \\
\mathrm{C}(46) \mathrm{C}(47) \mathrm{C}(48) \mathrm{O}(6) \mathrm{O}(7)\end{array}$ & Hfa3 & 0.022 & $C(46)$ \\
\hline
\end{tabular}


Table S19. Selected least-squares planes data of complex [L3La((hfa $\left.)_{3}\right]$.

\begin{tabular}{lccc}
\hline & Abbreviation & $\begin{array}{c}\text { Max. } \\
\text { deviation }(\AA)\end{array}$ & Atom \\
\hline $\begin{array}{l}\text { Pyridine } \\
\text { N(1) C(1) C(2) C(3) C(4) C(5) }\end{array}$ & Py & 0.006 & $\mathrm{C}(4)$ \\
$\begin{array}{l}\text { Benzimidazole 1 } \\
\text { C(6) N(2) C(7) C(8) C(9) C(10) C(11) C(12) N(3) }\end{array}$ & Bz1 & 0.014 & $\mathrm{~N}(2)$ \\
$\begin{array}{l}\text { Benzimidazole 2 } \\
\text { C(18) N(4) C(19) C(20) C(21) C(22) C(23) C(24) N(5) }\end{array}$ & Bz2 & 0.015 & $\mathrm{C}(22)$ \\
$\begin{array}{l}\text { Hexafluoroacetylacetonate } \\
\text { C(36) C(37) C(38) O(2) O(3) }\end{array}$ & $\mathrm{Hfa}$ & 0.044 & $\mathrm{C}(36)$ \\
$\begin{array}{l}\text { Hexafluoroacetylacetonate } \\
\text { C(41) C(42) C(43) O(4) O(5) }\end{array}$ & $\mathrm{Hfa} 2$ & 0.013 & $\mathrm{C}(43)$ \\
$\begin{array}{l}\text { Hexafluoroacetylacetonate } \\
\text { C(46) C(47) C(48) O(6) O(7) }\end{array}$ & & & \\
\hline
\end{tabular}


Table S20. Interplanar angles for $\left[\mathbf{L} \boldsymbol{k E u}(\mathrm{hfa})_{3}\right](\boldsymbol{k}=3-5)$

\begin{tabular}{|c|c|c|c|c|c|c|c|c|c|c|c|c|c|c|c|}
\hline \multicolumn{16}{|c|}{ Interplanar angles $\left[\mathbf{L} \boldsymbol{k E u}(\mathrm{hfa})_{3}\right]\left(^{\circ}\right)$} \\
\hline Plane & \multicolumn{3}{|c|}{ Benzimidazole (1) } & \multicolumn{3}{|c|}{ Benzimidazole (2) } & \multicolumn{3}{|c|}{ Hfal } & \multicolumn{3}{|c|}{$\mathrm{Hfa} 2$} & \multicolumn{3}{|c|}{ Hfa3 } \\
\hline Compound & EuL5 & EuL4 & EuL3 & EuL5 & EuL4 & $\mathrm{EuL3}$ & EuL5 & EuL4 & EuL3 & EuL5 & EuL4 & $\mathrm{EuL3}$ & EuL5 & EuL4 & EuL3 \\
\hline pyridine & 13.37 & 16.11 & 17.05 & 35.15 & 35.72 & 38.58 & 81.35 & 81.0 & 79.34 & 45.71 & 48.0 & 50.57 & 46.07 & 43.7 & 42.56 \\
\hline Benzimidazole (1) & & & & 28.11 & 27.23 & 27.46 & 86.85 & 88.3 & 87.62 & 43.16 & 44.6 & 44.83 & 58.79 & 58.7 & 57.94 \\
\hline Benzimidazole $(2)^{a}$ & & & & & & & 65.08 & 64.8 & 64.92 & 18.11 & 19.4 & 20.06 & 65.18 & 62.8 & 64.82 \\
\hline Hfa1 & & & & & & & & & & 53.21 & 51.3 & 50.1 & 81.22 & 81.9 & 82.90 \\
\hline $\mathrm{Hfa} 2$ & & & & & & & & & & & & & 59.79 & 58.7 & 60.73 \\
\hline
\end{tabular}

${ }^{a}$ Disorder in the structure of Benzimidazole2 plane.

Table S21. Interplanar angles for $\left[\mathbf{L 3} \operatorname{Ln}(\mathrm{hfa})_{3}\right](\mathrm{Ln}=\mathrm{La}, \mathrm{Eu}, \mathrm{Lu})$.

\begin{tabular}{|c|c|c|c|c|c|c|c|c|c|c|c|c|c|c|c|}
\hline \multicolumn{16}{|c|}{ Interplanar angles $\left[\mathbf{L} 3 \operatorname{Ln}(\mathrm{hfa})_{3}\right]\left(^{\circ}\right)$} \\
\hline Plane & \multicolumn{3}{|c|}{ Benzimidazole (1) } & \multicolumn{3}{|c|}{ Benzimidazole (2) } & \multicolumn{3}{|c|}{ Hfa1 } & \multicolumn{3}{|c|}{ Hfa2 } & \multicolumn{3}{|c|}{ Hfa3 } \\
\hline Compound & LuL3 & $\mathrm{EuL3}$ & $\mathrm{LaL3}$ & LuL3 & $\mathrm{EuL3}$ & LaL3 & LuL3 & EuL3 & LaL3 & LuL3 & $\mathrm{EuL3}$ & LaL3 & LuL3 & $\mathrm{EuL3}$ & $\mathrm{LaL3}$ \\
\hline pyridine & 16.28 & 17.05 & 32.67 & 38.02 & 38.58 & 16.86 & 78.60 & 79.34 & 61.89 & 48.94 & 50.57 & 74.02 & 45.72 & 42.56 & 38.91 \\
\hline Benzimidazole (1) & & & & 27.04 & 27.46 & 27.46 & 87.03 & 87.62 & 56.87 & 43.48 & 44.83 & 88.60 & 59.76 & 57.94 & 57.01 \\
\hline Benzimidazole (2) & & & & & & & 65.93 & 64.92 & 73.39 & 19.89 & 20.06 & 66.52 & 65.22 & 64.82 & 55.47 \\
\hline Hfa1 & & & & & & & & & & 52.56 & 50.10 & 45.42 & 84.72 & 82.90 & 36.19 \\
\hline $\mathrm{Hfa} 2$ & & & & & & & & & & & & & 58.27 & 60.73 & 79.34 \\
\hline
\end{tabular}


Table S22. Selected bond distances $(\AA)$, in [L6( $\left.\left.\operatorname{Ln}(\mathrm{hfa})_{3}\right)_{2}\right],(\mathrm{Ln}=\mathrm{La}, \mathrm{Eu}, \mathrm{Yb}, \mathrm{Lu})$.

\begin{tabular}{lc}
\hline & $\begin{array}{c}\text { Selected Bond } \\
\text { Distances }(\AA)\end{array}$ \\
\cline { 2 - 2 } Atoms 1-2 & $2.5342(19)$ \\
\hline La-O(2) & $2.5296(16)$ \\
La-O(3) & 2.67 \\
La-O(4) & $2.4783(16)$ \\
La-O(5) & $2.5268(16)$ \\
La-O(6) & $2.5268(16)$ \\
La-O(7) & $2.5421(17)$ \\
La-N(1) & $2.7032(18)$ \\
La-N(2) & $2.6383(18)$ \\
La-N(4) & $2.6419(18)$ \\
\hline
\end{tabular}

\begin{tabular}{lc}
\hline & \multicolumn{2}{c}{$\begin{array}{c}\text { Selected Bond } \\
\text { Distances }(\AA)\end{array}$} \\
\cline { 2 - 2 } Atoms 1-2 & {$[\mathbf{L 6}(\mathrm{Eu}(\mathrm{hfa}) 3) 2]$} \\
\hline Eu-O(3) & $2.409(3)$ \\
$\mathrm{Eu}-\mathrm{O}(4)$ & $2.461(3)$ \\
$\mathrm{Eu}-\mathrm{O}(5)$ & $2.428(3)$ \\
$\mathrm{Eu}-\mathrm{O}(6)$ & $2.376(2)$ \\
$\mathrm{Eu}-\mathrm{O}(7)$ & $2.464(3)$ \\
Eu-O(8) & $2.432(3)$ \\
$\mathrm{Eu}-\mathrm{N}(1)$ & $2.583(3)$ \\
$\mathrm{Eu}-\mathrm{N}(4)$ & $2.543(3)$ \\
$\mathrm{Eu}-\mathrm{N}(5)$ & $2.528(3)$ \\
\hline
\end{tabular}

\begin{tabular}{lc}
\hline & $\begin{array}{c}\text { Selected Bond } \\
\text { Distances }(\AA)\end{array}$ \\
\cline { 2 - 2 } Atoms 1-2 & $2.314(4)$ \\
\hline $\mathrm{Lu}(\mathrm{Lu}(\mathrm{hfa}) 3) 2]$
\end{tabular}

\begin{tabular}{lc}
\hline Atoms 1-2 & $\begin{array}{c}\text { Selected Bond } \\
\text { Distances }(\AA)\end{array}$ \\
\cline { 2 - 2 }$\left[\mathbf{L 6} 6\left(\mathrm{Lu}(\mathrm{hfa})_{3}\right) 2\right]$ \\
\hline $\mathrm{Lu}(2)-\mathrm{O}(9)$ & $2.418(4)$ \\
$\mathrm{Lu}(2)-\mathrm{O}(10)$ & $2.321(3)$ \\
$\mathrm{Lu}(2)-\mathrm{O}(11)$ & $2.281(3)$ \\
$\mathrm{Lu}(2)-\mathrm{O}(12)$ & $2.309(4)$ \\
$\mathrm{Lu}(2)-\mathrm{O}(13)$ & $2.441(3)$ \\
$\mathrm{Lu}(2)-\mathrm{O}(14)$ & $2.324(4)$ \\
$\mathrm{Lu}(2)-\mathrm{N}(6)$ & $2.471(4)$ \\
$\mathrm{Lu}(2)-\mathrm{N}(8)$ & $2.505(4)$ \\
$\mathrm{Lu}(2)-\mathrm{N}(9)$ & $2.447(4)$ \\
\hline
\end{tabular}




\begin{tabular}{|c|c|c|c|}
\hline \multirow{2}{*}{ Atoms 1-2 } & $\begin{array}{l}\text { Selected Bond } \\
\text { Distances }(\AA)\end{array}$ & \multirow{2}{*}{ Atoms 1-2 } & $\begin{array}{l}\text { Selected Bond } \\
\text { Distances }(\AA)\end{array}$ \\
\hline & {$\left[\mathbf{L 6}\left(\mathrm{Yb}(\mathrm{hfa})_{3}\right)_{2}\right]$} & & {$\left[\mathbf{L 6} 6\left(\mathrm{Yb}(\mathrm{hfa})_{3}\right)_{2}\right]$} \\
\hline $\mathrm{Yb}(1)-\mathrm{O}(3)$ & $2.324(4)$ & $\mathrm{Yb}(2)-\mathrm{O}(9)$ & $2.466(5)$ \\
\hline $\mathrm{Yb}(1)-\mathrm{O}(4)$ & $2.442(4)$ & $\mathrm{Yb}(2)-\mathrm{O}(10)$ & $2.323(5)$ \\
\hline $\mathrm{Yb}(1)-\mathrm{O}(5)$ & $2.323(4)$ & $\mathrm{Yb}(2)-\mathrm{O}(11)$ & $2.428(5)$ \\
\hline $\mathrm{Yb}(1)-\mathrm{O}(6)$ & $2.289(4)$ & $\mathrm{Yb}(2)-\mathrm{O}(12)$ & $2.329(4)$ \\
\hline $\mathrm{Yb}(1)-\mathrm{O}(7)$ & $2.432(5)$ & $\mathrm{Yb}(2)-\mathrm{O}(13)$ & $2.296(4)$ \\
\hline $\mathrm{Yb}(1)-\mathrm{O}(8)$ & $2.320(4)$ & $\mathrm{Yb}(2)-\mathrm{O}(14)$ & $2.291(5)$ \\
\hline $\mathrm{Yb}(1)-\mathrm{N}(1)$ & $2.477(5)$ & $\mathrm{Yb}(2)-\mathrm{N}(6)$ & $2.500(5)$ \\
\hline $\mathrm{Yb}(1)-\mathrm{N}(3)$ & $2.494(5)$ & $\mathrm{Yb}(2)-\mathrm{N}(8)$ & $2.516(5)$ \\
\hline $\mathrm{Yb}(1)-\mathrm{N}(4)$ & $2.468(5)$ & $\mathrm{Yb}(2)-\mathrm{N}(9)$ & $2.478(5)$ \\
\hline
\end{tabular}

Table S23. Selected bond angles $\left(^{\circ}\right)$ in $\left[\mathbf{L 6}\left(\mathrm{La}(\mathrm{hfa})_{3}\right)_{2}\right]$.

\begin{tabular}{ll}
\hline Atoms 1-2-3 & Angles $\left(^{\circ}\right)$ \\
\hline $\mathrm{O}(2)-\mathrm{La}-\mathrm{O}(7)$ & $138.81(6)$ \\
$\mathrm{O}(2)-\mathrm{La}-\mathrm{N}(1)$ & $68.00(6)$ \\
$\mathrm{O}(2)-\mathrm{La}-\mathrm{N}(2)$ & $84.11(6)$ \\
$\mathrm{O}(2)-\mathrm{La}-\mathrm{N}(4)$ & $71.97(6)$ \\
$\mathrm{O}(7)-\mathrm{La}-\mathrm{N}(4)$ & $72.55(6)$ \\
$\mathrm{O}(3)-\mathrm{La}-\mathrm{O}(7)$ & $130.50(6)$ \\
$\mathrm{O}(3)-\mathrm{La}-\mathrm{N}(1)$ & $119.13(5)$ \\
$\mathrm{O}(3)-\mathrm{La}-\mathrm{N}(2)$ & $74.73(5)$ \\
$\mathrm{O}(3)-\mathrm{La}-\mathrm{N}(4)$ & $133.15(6)$ \\
$\mathrm{O}(4)-\mathrm{La}-\mathrm{O}(2)$ & $139.41(5)$ \\
$\mathrm{O}(4)-\mathrm{La}-\mathrm{O}(3)$ & $72.88(6)$ \\
$\mathrm{O}(4)-\mathrm{La}-\mathrm{O}(6)$ & $74.34(5)$ \\
$\mathrm{O}(4)-\mathrm{La}-\mathrm{O}(7)$ & $73.38(6)$ \\
$\mathrm{O}(4)-\mathrm{La}-\mathrm{N}(1)$ & $133.49(5)$ \\
$\mathrm{O}(4)-\mathrm{La}-\mathrm{N}(2)$ & $81.58(6)$ \\
\hline
\end{tabular}

\begin{tabular}{ll}
\hline Atoms 1-2-3 & Angles $\left(^{\circ}\right)$ \\
\hline $\mathrm{O}(5)-\mathrm{La}-\mathrm{O}(2)$ & $96.65(6)$ \\
$\mathrm{O}(5)-\mathrm{La}-\mathrm{O}(3)$ & $67.97(6)$ \\
$\mathrm{O}(5)-\mathrm{La}-\mathrm{O}(6)$ & $128.22(6)$ \\
$\mathrm{O}(5)-\mathrm{La}-\mathrm{O}(7)$ & $67.46(6)$ \\
$\mathrm{O}(5)-\mathrm{La}-\mathrm{N}(1)$ & $154.80(5)$ \\
$\mathrm{O}(5)-\mathrm{La}-\mathrm{N}(2)$ & $138.67(5)$ \\
$\mathrm{O}(5)-\mathrm{La}-\mathrm{N}(4)$ & $96.58(5)$ \\
$\mathrm{O}(6)-\mathrm{La}-\mathrm{O}(2)$ & $133.98(6)$ \\
$\mathrm{O}(6)-\mathrm{La}-\mathrm{O}(3)$ & $134.01(6)$ \\
$\mathrm{O}(6)-\mathrm{La}-\mathrm{N}(1)$ & $66.46(5)$ \\
$\mathrm{O}(6)-\mathrm{La}-\mathrm{N}(2)$ & $69.31(6)$ \\
$\mathrm{O}(6)-\mathrm{La}-\mathrm{N}(4)$ & $90.96(6)$ \\
$\mathrm{O}(7)-\mathrm{La}-\mathrm{N}(1)$ & $110.37(5)$ \\
$\mathrm{O}(7)-\mathrm{La}-\mathrm{N}(2)$ & $133.37(6)$ \\
$\mathrm{O}(7)-\mathrm{La}-\mathrm{N}(4)$ & $72.55(6)$ \\
\hline
\end{tabular}


Table S24. Selected bond angles $\left(^{\circ}\right)$ in $\left[\mathbf{L 6}\left(\operatorname{Eu}(\mathrm{hfa})_{3}\right)_{2}\right]$.

\begin{tabular}{|c|c|c|c|}
\hline Atoms 1-2-3 & Angles $\left(^{\circ}\right)$ & Atoms 1-2-3 & Angles $\left({ }^{\circ}\right)$ \\
\hline $\mathrm{O}(3)-\mathrm{Eu}(1)-\mathrm{O}(5)$ & $92.10(10)$ & $\mathrm{O}(5)-\mathrm{Eu}(1)-\mathrm{N}(5)$ & $135.73(9)$ \\
\hline $\mathrm{O}(3)-\mathrm{Eu}(1)-\mathrm{O}(7)$ & $134.01(9)$ & $\mathrm{O}(6)-\mathrm{Eu}(1)-\mathrm{O}(3)$ & $141.54(9)$ \\
\hline $\mathrm{O}(3)-\mathrm{Eu}(1)-\mathrm{O}(8)$ & $136.75(10)$ & $\mathrm{O}(6)-\mathrm{Eu}(1)-\mathrm{O}(4)$ & $72.62(9)$ \\
\hline $\mathrm{O}(3)-\mathrm{Eu}(1)-\mathrm{N}(1)$ & $68.74(10)$ & $\mathrm{O}(6)-\mathrm{Eu}(1)-\mathrm{O}(7)$ & $74.48(9)$ \\
\hline $\mathrm{O}(3)-\mathrm{Eu}(1)-\mathrm{N}(4)$ & $69.92(10)$ & $\mathrm{O}(6)-\mathrm{Eu}(1)-\mathrm{O}(8)$ & $70.94(9)$ \\
\hline $\mathrm{O}(3)-\mathrm{Eu}(1)-\mathrm{N}(5)$ & $86.24(10)$ & $\mathrm{O}(6)-\mathrm{Eu}(1)-\mathrm{N}(1)$ & $132.48(9)$ \\
\hline $\mathrm{O}(4)-\mathrm{Eu}(1)-\mathrm{O}(7)$ & $129.49(9)$ & $\mathrm{O}(6)-\mathrm{Eu}(1)-\mathrm{N}(4)$ & $144.59(9)$ \\
\hline $\mathrm{O}(4)-\mathrm{Eu}(1)-\mathrm{N}(1)$ & $119.36(10)$ & $\mathrm{O}(6)-\mathrm{Eu}(1)-\mathrm{N}(5)$ & $80.37(9)$ \\
\hline $\mathrm{O}(4)-\mathrm{Eu}(1)-\mathrm{N}(4)$ & $132.76(9)$ & $\mathrm{O}(7)-\mathrm{Eu}(1)-\mathrm{N}(1)$ & $111.15(9)$ \\
\hline $\mathrm{O}(4)-\mathrm{Eu}(1)-\mathrm{N}(5)$ & $72.23(10)$ & $\mathrm{O}(7)-\mathrm{Eu}(1)-\mathrm{N}(4)$ & $70.11(9)$ \\
\hline $\mathrm{O}(5)-\mathrm{Eu}(1)-\mathrm{O}(4)$ & $66.05(9)$ & $\mathrm{O}(7)-\mathrm{Eu}(1)-\mathrm{N}(5)$ & $136.87(9)$ \\
\hline $\mathrm{O}(5)-\mathrm{Eu}(1)-\mathrm{O}(7)$ & $68.63(9)$ & $\mathrm{O}(8)-\mathrm{Eu}(1)-\mathrm{O}(4)$ & $130.88(9)$ \\
\hline $\mathrm{O}(5)-\mathrm{Eu}(1)-\mathrm{O}(8)$ & $130.16(10)$ & $\mathrm{O}(8)-\mathrm{Eu}(1)-\mathrm{N}(1)$ & $68.36(9)$ \\
\hline $\mathrm{O}(5)-\mathrm{Eu}(1)-\mathrm{N}(1)$ & $153.50(9)$ & $\mathrm{O}(8)-\mathrm{Eu}(1)-\mathrm{N}(4)$ & $95.33(9)$ \\
\hline $\mathrm{O}(5)-\mathrm{Eu}(1)-\mathrm{N}(4)$ & $93.45(9)$ & $\mathrm{O}(8)-\mathrm{Eu}(1)-\mathrm{N}(5)$ & $70.17(10)$ \\
\hline
\end{tabular}


Table S25. Selected bond angles $\left(^{\circ}\right)$ in $\left[\mathbf{L 6}\left(\mathrm{Lu}(\mathrm{hfa})_{3}\right)_{2}\right]$.

\begin{tabular}{ll|lc}
\hline Atoms 1-2-3 & Angles ( ${ }^{\circ}$ & \multicolumn{1}{|c}{ Atoms 1-2-3 } & Angles $\left(^{\circ}\right)$ \\
\hline $\mathrm{O}(3)-\mathrm{Lu}(1)-\mathrm{N}(1)$ & $72.07(15)$ & $\mathrm{O}(8)-\mathrm{Lu}(1)-\mathrm{N}(1)$ & $69.90(15)$ \\
$\mathrm{O}(3)-\mathrm{Lu}(1)-\mathrm{N}(3)$ & $68.50(12)$ & $\mathrm{O}(8)-\mathrm{Lu}(1)-\mathrm{N}(3)$ & $113.33(13)$ \\
$\mathrm{O}(3)-\mathrm{Lu}(1)-\mathrm{N}(4)$ & $88.55(13)$ & $\mathrm{O}(8)-\mathrm{Lu}(1)-\mathrm{N}(4)$ & $134.49(13)$ \\
$\mathrm{O}(3)-\mathrm{Lu}(1)-\mathrm{O}(5)$ & $137.13(13)$ & $\mathrm{O}(8)-\mathrm{Lu}(1)-\mathrm{O}(4)$ & $126.65(14)$ \\
$\mathrm{O}(3)-\mathrm{Lu}(1)-\mathrm{O}(7)$ & $138.95(12)$ & $\mathrm{O}(9)-\mathrm{Lu}(2)-\mathrm{N}(6)$ & $131.18(13)$ \\
$\mathrm{O}(3)-\mathrm{Lu}(1)-\mathrm{O}(8)$ & $135.42(15)$ & $\mathrm{O}(9)-\mathrm{Lu}(2)-\mathrm{N}(8)$ & $114.03(12)$ \\
$\mathrm{O}(4)-\mathrm{Lu}(1)-\mathrm{N}(1)$ & $134.90(14)$ & $\mathrm{O}(9)-\mathrm{Lu}(2)-\mathrm{N}(9)$ & $69.93(13)$ \\
$\mathrm{O}(4)-\mathrm{Lu}(1)-\mathrm{N}(3)$ & $120.00(13)$ & $\mathrm{O}(9)-\mathrm{Lu}(2)-\mathrm{O}(13)$ & $128.02(12)$ \\
$\mathrm{O}(4)-\mathrm{Lu}(1)-\mathrm{N}(4)$ & $71.55(12)$ & $\mathrm{O}(10)-\mathrm{Lu}(2)-\mathrm{N}(6)$ & $68.62(12)$ \\
$\mathrm{O}(5)-\mathrm{Lu}(1)-\mathrm{N}(1)$ & $138.17(14)$ & $\mathrm{O}(10)-\mathrm{Lu}(2)-\mathrm{N}(8)$ & $69.44(12)$ \\
$\mathrm{O}(5)-\mathrm{Lu}(1)-\mathrm{N}(3)$ & $143.22(12)$ & $\mathrm{O}(10)-\mathrm{Lu}(2)-\mathrm{N}(9)$ & $95.43(13)$ \\
$\mathrm{O}(5)-\mathrm{Lu}(1)-\mathrm{O}(4)$ & $67.88(13)$ & $\mathrm{O}(10)-\mathrm{Lu}(2)-\mathrm{O}(13)$ & $128.76(11)$ \\
$\mathrm{O}(5)-\mathrm{Lu}(1)-\mathrm{O}(8)$ & $69.38(13)$ & $\mathrm{O}(10)-\mathrm{Lu}(2)-\mathrm{O}(14)$ & $135.90(12)$ \\
$\mathrm{O}(6)-\mathrm{Lu}(1)-\mathrm{N}(1)$ & $80.36(15)$ & $\mathrm{O}(11)-\mathrm{Lu}(2)-\mathrm{N}(6)$ & $83.68(11)$ \\
$\mathrm{O}(6)-\mathrm{Lu}(1)-\mathrm{N}(3)$ & $138.66(14)$ & $\mathrm{O}(11)-\mathrm{Lu}(2)-\mathrm{N}(8)$ & $141.11(12)$ \\
$\mathrm{O}(6)-\mathrm{Lu}(1)-\mathrm{N}(4)$ & $141.53(15)$ & $\mathrm{O}(11)-\mathrm{Lu}(2)-\mathrm{N}(9)$ & $139.40(13)$ \\
$\mathrm{O}(6)-\mathrm{Lu}(1)-\mathrm{O}(3)$ & $80.39(17)$ & $\mathrm{O}(11)-\mathrm{Lu}(2)-\mathrm{O}(9)$ & $70.07(12)$ \\
$\mathrm{O}(6)-\mathrm{Lu}(1)-\mathrm{O}(4)$ & $70.04(16)$ & $\mathrm{O}(11)-\mathrm{Lu}(2)-\mathrm{O}(10)$ & $77.12(12)$ \\
$\mathrm{O}(6)-\mathrm{Lu}(1)-\mathrm{O}(7)$ & $138.28(16)$ & $\mathrm{O}(11)-\mathrm{Lu}(2)-\mathrm{O}(13)$ & $69.51(12)$ \\
$\mathrm{O}(6)-\mathrm{Lu}(1)-\mathrm{O}(8)$ & $71.17(18)$ & $\mathrm{O}(11)-\mathrm{Lu}(2)-\mathrm{O}(14)$ & $139.03(12)$ \\
$\mathrm{O}(7)-\mathrm{Lu}(1)-\mathrm{N}(1)$ & $97.40(14)$ & $\mathrm{O}(12)-\mathrm{Lu}(2)-\mathrm{N}(6)$ & $139.56(12)$ \\
$\mathrm{O}(7)-\mathrm{Lu}(1)-\mathrm{N}(3)$ & $71.14(11)$ & $\mathrm{O}(12)-\mathrm{Lu}(2)-\mathrm{N}(8)$ & $141.15(13)$ \\
$\mathrm{O}(7)-\mathrm{Lu}(1)-\mathrm{N}(4)$ & $68.27(11)$ & $\mathrm{O}(12)-\mathrm{Lu}(2)-\mathrm{N}(9)$ & $82.95(14)$ \\
$\mathrm{O}(7)-\mathrm{Lu}(1)-\mathrm{O}(4)$ & $127.36(11)$ & $\mathrm{O}(12)-\mathrm{Lu}(2)-\mathrm{O}(9)$ & $70.73(13)$ \\
$\mathrm{O}(7)-\mathrm{Lu}(1)-\mathrm{O}(5)$ & $76.68(12)$ & $\mathrm{O}(12)-\mathrm{Lu}(2)-\mathrm{O}(10)$ & $138.44(12)$ \\
$\mathrm{O}(13)-\mathrm{Lu}(2)-\mathrm{N}(6)$ & $70.02(12)$ & $\mathrm{O}(12)-\mathrm{Lu}(2)-\mathrm{O}(13)$ & $69.88(12)$ \\
$\mathrm{O}(13)-\mathrm{Lu}(2)-\mathrm{N}(8)$ & $117.95(12)$ & $\mathrm{O}(12)-\mathrm{Lu}(2)-\mathrm{O}(14)$ & $83.11(13)$ \\
$\mathrm{O}(13)-\mathrm{Lu}(2)-\mathrm{N}(9)$ & $134.95(13)$ & $\mathrm{O}(14)-\mathrm{Lu}(2)-\mathrm{O}(9)$ & $135.53(12)$ \\
$\mathrm{O}(14)-\mathrm{Lu}(2)-\mathrm{N}(6)$ & $88.19(12)$ & $\mathrm{O}(14)-\mathrm{Lu}(2)-\mathrm{N}(9)$ & $71.72(13)$ \\
\hline
\end{tabular}


Table S26. Selected bond angles $\left(^{\circ}\right)$ in $\left[\mathbf{L 6}\left(\mathrm{Yb}(\mathrm{hfa})_{3}\right)_{2}\right]$.

\begin{tabular}{ll|ll}
\hline Atoms 1-2-3 & Angles ( $)$ & \multicolumn{1}{|c}{ Atoms 1-2-3 } & Angles $\left(^{\circ}\right)$ \\
\hline $\mathrm{O}(3)-\mathrm{Yb}(1)-\mathrm{N}(1)$ & $71.21(16)$ & $\mathrm{O}(8)-\mathrm{Yb}(1)-\mathrm{N}(1)$ & $95.80(16)$ \\
$\mathrm{O}(3)-\mathrm{Yb}(1)-\mathrm{N}(3)$ & $69.99(15)$ & $\mathrm{O}(8)-\mathrm{Yb}(1)-\mathrm{N}(3)$ & $71.43(15)$ \\
$\mathrm{O}(3)-\mathrm{Yb}(1)-\mathrm{N}(4)$ & $91.75(15)$ & $\mathrm{O}(8)-\mathrm{Yb}(1)-\mathrm{N}(4)$ & $68.73(15)$ \\
$\mathrm{O}(3)-\mathrm{Yb}(1)-\mathrm{O}(5)$ & $135.87(16)$ & $\mathrm{O}(8)-\mathrm{Yb}(1)-\mathrm{O}(4)$ & $128.10(14)$ \\
$\mathrm{O}(3)-\mathrm{Yb}(1)-\mathrm{O}(7)$ & $132.82(16)$ & $\mathrm{O}(9)-\mathrm{Yb}(2)-\mathrm{N}(6)$ & $69.88(15)$ \\
$\mathrm{O}(3)-\mathrm{Yb}(1)-\mathrm{O}(8)$ & $141.22(15)$ & $\mathrm{O}(9)-\mathrm{Yb}(2)-\mathrm{N}(8)$ & $118.24(15)$ \\
$\mathrm{O}(4)-\mathrm{Yb}(1)-\mathrm{N}(1)$ & $135.43(16)$ & $\mathrm{O}(9)-\mathrm{Yb}(2)-\mathrm{N}(9)$ & $135.17(17)$ \\
$\mathrm{O}(4)-\mathrm{Yb}(1)-\mathrm{N}(3)$ & $117.46(14)$ & $\mathrm{O}(9)-\mathrm{Yb}(2)-\mathrm{O}(13)$ & $71.07(16)$ \\
$\mathrm{O}(4)-\mathrm{Yb}(1)-\mathrm{N}(4)$ & $70.43(15)$ & $\mathrm{O}(10)-\mathrm{Yb}(2)-\mathrm{N}(6)$ & $87.46(17)$ \\
$\mathrm{O}(5)-\mathrm{Yb}(1)-\mathrm{N}(1)$ & $139.16(16)$ & $\mathrm{O}(10)-\mathrm{Yb}(2)-\mathrm{N}(8)$ & $67.23(17)$ \\
$\mathrm{O}(5)-\mathrm{Yb}(1)-\mathrm{N}(3)$ & $143.13(16)$ & $\mathrm{O}(10)-\mathrm{Yb}(2)-\mathrm{N}(9)$ & $72.22(17)$ \\
$\mathrm{O}(5)-\mathrm{Yb}(1)-\mathrm{O}(4)$ & $68.33(16)$ & $\mathrm{O}(10)-\mathrm{Yb}(2)-\mathrm{O}(13)$ & $83.09(19)$ \\
$\mathrm{O}(5)-\mathrm{Yb}(1)-\mathrm{O}(8)$ & $77.48(17)$ & $\mathrm{O}(10)-\mathrm{Yb}(2)-\mathrm{O}(14)$ & $138.03(17)$ \\
$\mathrm{O}(6)-\mathrm{Yb}(1)-\mathrm{N}(1)$ & $81.78(17)$ & $\mathrm{O}(11)-\mathrm{Yb}(2)-\mathrm{N}(6)$ & $134.00(17)$ \\
$\mathrm{O}(6)-\mathrm{Yb}(1)-\mathrm{N}(3)$ & $139.55(16)$ & $\mathrm{O}(11)-\mathrm{Yb}(2)-\mathrm{N}(8)$ & $113.05(16)$ \\
$\mathrm{O}(6)-\mathrm{Yb}(1)-\mathrm{N}(4)$ & $141.30(16)$ & $\mathrm{O}(11)-\mathrm{Yb}(2)-\mathrm{N}(9)$ & $70.30(18)$ \\
$\mathrm{O}(6)-\mathrm{Yb}(1)-\mathrm{O}(3)$ & $78.35(16)$ & $\mathrm{O}(11)-\mathrm{Yb}(2)-\mathrm{O}(9)$ & $128.68(16)$ \\
$\mathrm{O}(6)-\mathrm{Yb}(1)-\mathrm{O}(4)$ & $71.09(16)$ & $\mathrm{O}(11)-\mathrm{Yb}(2)-\mathrm{O}(10)$ & $136.74(17)$ \\
$\mathrm{O}(6)-\mathrm{Yb}(1)-\mathrm{O}(7)$ & $70.23(17)$ & $\mathrm{O}(11)-\mathrm{Yb}(2)-\mathrm{O}(13)$ & $71.15(18)$ \\
$\mathrm{O}(6)-\mathrm{Yb}(1)-\mathrm{O}(8)$ & $137.37(16)$ & $\mathrm{O}(11)-\mathrm{Yb}(2)-\mathrm{O}(14)$ & $69.85(16)$ \\
$\mathrm{O}(7)-\mathrm{Yb}(1)-\mathrm{N}(1)$ & $70.23(17)$ & $\mathrm{O}(12)-\mathrm{Yb}(2)-\mathrm{N}(6)$ & $68.29(16)$ \\
$\mathrm{O}(7)-\mathrm{Yb}(1)-\mathrm{N}(3)$ & $114.89(16)$ & $\mathrm{O}(12)-\mathrm{Yb}(2)-\mathrm{N}(8)$ & $69.77(15)$ \\
$\mathrm{O}(7)-\mathrm{Yb}(1)-\mathrm{N}(4)$ & $134.37(15)$ & $\mathrm{O}(12)-\mathrm{Yb}(2)-\mathrm{N}(9)$ & $96.10(17)$ \\
$\mathrm{O}(7)-\mathrm{Yb}(1)-\mathrm{O}(4)$ & $127.64(17)$ & $\mathrm{O}(12)-\mathrm{Yb}(2)-\mathrm{O}(9)$ & $127.81(15)$ \\
$\mathrm{O}(7)-\mathrm{Yb}(1)-\mathrm{O}(5)$ & $69.80(16)$ & $\mathrm{O}(12)-\mathrm{Yb}(2)-\mathrm{O}(10)$ & $136.29(16)$ \\
$\mathrm{O}(13)-\mathrm{Yb}(2)-\mathrm{N}(6)$ & $140.69(17)$ & $\mathrm{O}(12)-\mathrm{Yb}(2)-\mathrm{O}(13)$ & $138.22(17)$ \\
$\mathrm{O}(13)-\mathrm{Yb}(2)-\mathrm{N}(8)$ & $140.19(18)$ & $\mathrm{O}(12)-\mathrm{Yb}(2)-\mathrm{O}(14)$ & $77.36(16)$ \\
$\mathrm{O}(13)-\mathrm{Yb}(2)-\mathrm{N}(9)$ & $82.08(19)$ & $\mathrm{O}(14)-\mathrm{Yb}(2)-\mathrm{O}(9)$ & $69.07(16)$ \\
$\mathrm{O}(14)-\mathrm{Yb}(2)-\mathrm{N}(6)$ & $84.71(15)$ & $\mathrm{O}(14)-\mathrm{Yb}(2)-\mathrm{N}(9)$ & $139.26(17)$ \\
\hline
\end{tabular}


Table S27 Selected least-squares planes data of complex [L6( $\left.\left.\mathrm{La}(\mathrm{hfa})_{3}\right)_{2}\right]$.

\begin{tabular}{|c|c|c|c|}
\hline plane & Abbreviation & $\begin{array}{c}\text { Max. } \\
\text { deviation }(\AA)\end{array}$ & Atom \\
\hline $\begin{array}{l}\text { Benzimidazole } 1 \\
C(18) \mathrm{N}(3) \mathrm{C}(23) \mathrm{C}(20) \mathrm{C}(22) \mathrm{C}(19) \mathrm{C}(24) \mathrm{C}(21) \mathrm{N}(2)\end{array}$ & Bz1 & 0.009 & $\mathrm{~N}(2)$ \\
\hline $\begin{array}{l}\text { Pyridine } 1 \\
\mathrm{~N}(1) \mathrm{C}(1) \mathrm{C}(2) \mathrm{C}(3) \mathrm{C}(4) \mathrm{C}(5)\end{array}$ & Py1 & 0.007 & $\mathrm{C}(1)$ \\
\hline $\begin{array}{l}\text { Benzimidazole } 2^{a} \\
\mathrm{C}(6) \mathrm{N}(4) \mathrm{C}(11) \mathrm{C}(8 \mathrm{~B}) \mathrm{C}(10) \mathrm{C}(7 \mathrm{~B}) \mathrm{C}(12) \mathrm{C}(9 \mathrm{~B}) \\
\mathrm{N}(5 \mathrm{~B}) \\
\mathrm{C}(6) \mathrm{N}(4) \mathrm{C}(11) \mathrm{C}(8 \mathrm{~A}) \mathrm{C}(10) \mathrm{C}(7 \mathrm{~A}) \mathrm{C}(12) \mathrm{C}(9 \mathrm{~A}) \\
\mathrm{N}(5 \mathrm{~A})\end{array}$ & $\mathrm{Bz} 2$ & $\begin{array}{c}0.08 \\
0.107\end{array}$ & $\begin{array}{l}\mathrm{C}(10) \\
\mathrm{C}(6)\end{array}$ \\
\hline $\begin{array}{l}\text { Hexafluoroacetylacetonate } 1 \\
\mathrm{C}(37) \mathrm{C}(38) \mathrm{C}(36) \mathrm{O}(3) \mathrm{O}(2)\end{array}$ & Hfa1 & 0.054 & $\mathrm{C}(38)$ \\
\hline $\begin{array}{l}\text { Hexafluoroacetylacetonate } 2 \\
\mathrm{C}(41) \mathrm{C}(42) \mathrm{C}(43) \mathrm{O}(5) \mathrm{O}(4)\end{array}$ & $\mathrm{Hfa} 2$ & 0.007 & $\mathrm{C}(43)$ \\
\hline $\begin{array}{l}\text { Hexafluoroacetylacetonate } 3 \\
\mathrm{C}(46) \mathrm{C}(47) \mathrm{C}(48) \mathrm{O}(6) \mathrm{O}(7)\end{array}$ & $\mathrm{Hfa} 3$ & 0.031 & $\mathrm{C}(48)$ \\
\hline
\end{tabular}

${ }^{a}$ Disorder in the structure of Benzimidazole 2 plane.

Table S28. Selected least-squares planes data of complex [L6(Eu(hfa)3 $\left.)_{2}\right]$.

\begin{tabular}{|l|c|c|c|}
\hline \multicolumn{1}{|c|}{ plane } & Abbreviation & $\begin{array}{c}\text { Max. } \\
\text { deviation }(\AA)\end{array}$ & Atom \\
\hline $\begin{array}{l}\text { Benzimidazole 1 } \\
\mathrm{C}(6) \mathrm{N}(3) \mathrm{C}(8) \mathrm{C}(11) \mathrm{C}(7) \mathrm{C}(10) \mathrm{C}(12) \mathrm{C}(9) \mathrm{N}(4)\end{array}$ & $\mathrm{Bz} 1$ & 0.024 & $\mathrm{C}(6)$ \\
\hline $\begin{array}{l}\text { Pyridine 1 } \\
\mathrm{N}(1) \mathrm{C}(1) \mathrm{C}(2) \mathrm{C}(3) \mathrm{C}(4) \mathrm{C}(5)\end{array}$ & Py1 & -0.010 & $\mathrm{C}(5)$ \\
\hline $\begin{array}{l}\text { Benzimidazole 2 } \\
\mathrm{C}(19) \mathrm{N}(5) \mathrm{C}(20) \mathrm{C}(21) \mathrm{C}(22) \mathrm{C}(23) \mathrm{C}(24) \mathrm{C}(18) \mathrm{N}(6)\end{array}$ & $\mathrm{Bz2}$ & -0.012 & $\mathrm{C}(22)$ \\
\hline $\begin{array}{l}\text { Hexafluoroacetylacetonate 1 } \\
\mathrm{C}(37) \mathrm{C}(38) \mathrm{C}(36) \mathrm{O}(3) \mathrm{O}(4)\end{array}$ & $\mathrm{Hfa} 1$ & 0.040 & $\mathrm{C}(38)$ \\
\hline $\begin{array}{l}\text { Hexafluoroacetylacetonate 2 } \\
\mathrm{C}(41) \mathrm{C}(42) \mathrm{C}(43) \mathrm{O}(5) \mathrm{O}(6)\end{array}$ & Hfa2 & 0.040 & $\mathrm{C}(41)$ \\
\hline $\begin{array}{l}\text { Hexafluoroacetylacetonate } 3 \\
\mathrm{C}(46) \mathrm{C}(47) \mathrm{C}(48) \text { O(8) O(7) }\end{array}$ & Hfa3 & -0.035 & $\mathrm{C}(46)$ \\
\hline
\end{tabular}


Table S29. Selected least-squares planes data of complex [L6(Lu(hfa $\left.\left.)_{3}\right)_{2}\right]$.

\begin{tabular}{|c|c|c|c|}
\hline plane & Abbreviation & $\begin{array}{l}\text { Max. deviation } \\
(\AA)\end{array}$ & Atom \\
\hline $\begin{array}{l}\text { Benzimidazole } 1 \\
C(1) C(2) C(3) C(4) C(5) C(6) C(7) N(1) N(1)\end{array}$ & Bz1 & 0.024 & $C(2)$ \\
\hline $\begin{array}{l}\text { Pyridine } 1 \\
\mathrm{~N}(3) \mathrm{C}(13) \mathrm{C}(14) \mathrm{C}(15) \mathrm{C}(16) \mathrm{C}(17)\end{array}$ & Py1 & 0.008 & $\mathrm{C}(14)$ \\
\hline $\begin{array}{l}\text { Benzimidazole } 2 \\
C(24) C(25) C(26) C(27) C(28) C(29) C(30) N(4) N(5)\end{array}$ & $\mathrm{Bz} 2$ & 0.029 & $C(25)$ \\
\hline $\begin{array}{l}\text { Benzimidazole } 3 \\
C(36) C(37) C(38) C(39) C(40) C(41) C(42) N(6) N(7)\end{array}$ & $\mathrm{Bz} 3$ & 0.020 & $\mathrm{~N}(6)$ \\
\hline $\begin{array}{l}\text { Pyridine } 2 \\
\mathrm{~N}(8) \mathrm{C}(48) \mathrm{C}(49) \mathrm{C}(50) \mathrm{C}(51) \mathrm{C}(52)\end{array}$ & Py2 & 0.008 & $\mathrm{~N}(8)$ \\
\hline $\begin{array}{l}\text { Benzimidazole } 4 \\
C(59) C(60) C(61) C(62) C(63) C(64) C(65) N(9) N(10)\end{array}$ & $\mathrm{Bz} 4$ & 0.016 & $\mathrm{C}(61)$ \\
\hline $\begin{array}{l}\text { Hexafluoroacetylacetonate } 1 \\
\mathrm{C}(1 \mathrm{H}) \mathrm{C}(2 \mathrm{H}) \mathrm{C}(3 \mathrm{H}) \mathrm{O}(3) \mathrm{O}(4)\end{array}$ & Hfal & 0.027 & $\mathrm{C}(3 \mathrm{H})$ \\
\hline $\begin{array}{l}\text { Hexafluoroacetylacetonate } 2 \\
\mathrm{C}(6 \mathrm{H}) \mathrm{C}(7 \mathrm{H}) \mathrm{C}(8 \mathrm{H}) \mathrm{O}(5) \mathrm{O}(6)\end{array}$ & $\mathrm{Hfa} 2$ & 0.038 & $\mathrm{C}(6 \mathrm{H})$ \\
\hline $\begin{array}{l}\text { Hexafluoroacetylacetonate } 3 \\
\mathrm{C}(11 \mathrm{H}) \mathrm{C}(12 \mathrm{H}) \mathrm{C}(13 \mathrm{H}) \mathrm{O}(7) \mathrm{O}(8)\end{array}$ & Hfa3 & 0.025 & $\mathrm{C}(13 \mathrm{H})$ \\
\hline $\begin{array}{l}\text { Hexafluoroacetylacetonate } 4 \\
\mathrm{C}(16 \mathrm{H}) \mathrm{C}(17 \mathrm{H}) \mathrm{C}(18 \mathrm{H}) \mathrm{O}(9) \mathrm{O}(10)\end{array}$ & Hfa4 & 0.023 & $\mathrm{C}(16 \mathrm{H})$ \\
\hline $\begin{array}{l}\text { Hexafluoroacetylacetonate } 5 \\
\mathrm{C}(21 \mathrm{H}) \mathrm{C}(22 \mathrm{H}) \mathrm{C}(23 \mathrm{H}) \mathrm{O}(11) \mathrm{O}(12)\end{array}$ & Hfa5 & 0.020 & $\mathrm{C}(21 \mathrm{H})$ \\
\hline $\begin{array}{l}\text { Hexafluoroacetylacetonate } 6 \\
\mathrm{C}(26 \mathrm{H}) \mathrm{C}(27 \mathrm{H}) \mathrm{C}(28 \mathrm{H}) \mathrm{O}(13) \mathrm{O}(14)\end{array}$ & Hfa6 & 0.046 & $\mathrm{C}(26 \mathrm{H})$ \\
\hline
\end{tabular}


Table S30. Selected least-squares planes data of complex $\left[\mathbf{L 6}\left(\mathrm{Yb}(\mathrm{hfa})_{3}\right)_{2}\right]$.

\begin{tabular}{|c|c|c|c|}
\hline plane & Abbreviation & $\begin{array}{c}\text { Max. } \\
\text { deviation }(\AA)\end{array}$ & Atom \\
\hline $\begin{array}{l}\text { Benzimidazole } 1 \\
C(1) C(2) C(3) C(4) C(5) C(6) C(7) N(1) N(1)\end{array}$ & Bz1 & 0.012 & $\mathrm{C}(4)$ \\
\hline $\begin{array}{l}\text { Pyridine } 1 \\
\mathrm{~N}(3) \mathrm{C}(13) \mathrm{C}(14) \mathrm{C}(15) \mathrm{C}(16) \mathrm{C}(17)\end{array}$ & Py1 & 0.013 & $\mathrm{C}(17,14)$ \\
\hline $\begin{array}{l}\text { Benzimidazole } 2 \\
C(24) C(25) C(26) C(27) C(28) C(29) C(30) N(4) N(5)\end{array}$ & $\mathrm{Bz} 2$ & 0.021 & $\mathrm{~N}(4)$ \\
\hline $\begin{array}{l}\text { Benzimidazole } 3 \\
C(36) C(37) C(38) C(39) C(40) C(41) C(42) N(6) N(7)\end{array}$ & $\mathrm{Bz} 3$ & 0.018 & $\mathrm{~N}(6)$ \\
\hline $\begin{array}{l}\text { Pyridine } 2 \\
\mathrm{~N}(8) \mathrm{C}(48) \mathrm{C}(49) \mathrm{C}(50) \mathrm{C}(51) \mathrm{C}(52)\end{array}$ & Py 2 & 0.012 & $\mathrm{C}(50,51)$ \\
\hline $\begin{array}{l}\text { Benzimidazole } 4 \\
C(59) C(60) C(61) C(62) C(63) C(64) C(65) N(9) N(10)\end{array}$ & $\mathrm{Bz} 4$ & 0.007 & $\mathrm{~N}(10)$ \\
\hline $\begin{array}{l}\text { Hexafluoroacetylacetonate } 1 \\
\mathrm{C}(72) \mathrm{C}(73) \mathrm{C}(74) \mathrm{O}(3) \mathrm{O}(4)\end{array}$ & Hfa1 & 0.025 & $\mathrm{C}(74)$ \\
\hline $\begin{array}{l}\text { Hexafluoroacetylacetonate } 2 \\
\mathrm{C}(78) \mathrm{C}(79) \mathrm{C}(77) \mathrm{O}(5) \mathrm{O}(6)\end{array}$ & $\mathrm{Hfa} 2$ & 0.023 & $C(77)$ \\
\hline $\begin{array}{l}\text { Hexafluoroacetylacetonate } 3 \\
\mathrm{C}(82) \mathrm{C}(83) \mathrm{C}(84) \mathrm{O}(7) \mathrm{O}(8)\end{array}$ & Hfa3 & 0.036 & $\mathrm{C}(82)$ \\
\hline $\begin{array}{l}\text { Hexafluoroacetylacetonate } 4 \\
\mathrm{C}(87) \mathrm{C}(88) \mathrm{C}(89) \mathrm{O}(9) \mathrm{O}(10)\end{array}$ & $\mathrm{Hfa} 4$ & 0.032 & $\mathrm{C}(87)$ \\
\hline $\begin{array}{l}\text { Hexafluoroacetylacetonate } 5 \\
\mathrm{C}(92) \mathrm{C}(93) \mathrm{C}(94) \mathrm{O}(11) \mathrm{O}(12)\end{array}$ & Hfa5 & 0.020 & $\mathrm{C}(92)$ \\
\hline $\begin{array}{l}\text { Hexafluoroacetylacetonate } 6 \\
\mathrm{C}(97) \mathrm{C}(98) \mathrm{C}(99) \mathrm{O}(13) \mathrm{O}(14)\end{array}$ & Hfa6 & 0.004 & $\mathrm{C}(97)$ \\
\hline
\end{tabular}


Table S31. Interplanar angles for [L6(La(hfa $\left.\left.)_{3}\right)_{2}\right]$.

\begin{tabular}{|c|c|c|c|c|c|}
\hline Plane & $\begin{array}{c}\text { Benzimidazole } \\
\text { (1) }\end{array}$ & $\begin{array}{c}\text { Benzimidazole } \\
\text { (2) }\end{array}$ & $\mathrm{Hfa}^{a}$ & $\mathrm{Hfa} 2$ & Hfa3 \\
\hline pyridine & $30.34(7)$ & $\begin{array}{l}13.13(7) \mathrm{A} \\
14.40(9) \mathrm{B}\end{array}$ & $78.29(9)$ & $26.40(7)$ & $72.45(9)$ \\
\hline Benzimidazole (1) & & $\begin{array}{l}41.63(6) \mathrm{A} \\
39.96(7) \mathrm{B}\end{array}$ & $107.25(9)$ & $21.30(8)$ & $79.59(8)$ \\
\hline Benzimidazole (2) & & & $\begin{array}{l}70.69(9) \mathrm{A} \\
67.30(9) \mathrm{B} \\
\end{array}$ & $\begin{array}{l}31.09(7) \mathrm{A} \\
40.60(8) \mathrm{B}\end{array}$ & $\begin{array}{c}63.4(1) \mathrm{A} \\
83.16(11) \mathrm{B} \\
\end{array}$ \\
\hline Hfa1 & & & & $78.24(9)$ & $86.13(7)$ \\
\hline $\mathrm{Hfa} 2$ & & & & & $58.9(1)$ \\
\hline
\end{tabular}

Table S32. Interplanar angles for [ $\left.\mathbf{L 6}\left(\mathrm{Eu}(\mathrm{hfa})_{3}\right)_{2}\right]$.

\begin{tabular}{|l|c|c|c|c|c|}
\hline \multicolumn{1}{|c|}{ Plane } & $\begin{array}{c}\text { Benzimidazole } \\
(1)\end{array}$ & $\begin{array}{c}\text { Benzimidazole } \\
(2)\end{array}$ & Hfa 1 & Hfa 2 & Hfa 3 \\
\hline pyridine & $11.67(12)$ & $15.31(12)$ & $96.0(2)$ & $30.70(13)$ & $72.20(17)$ \\
\hline Benzimidazole (1) & & $26.81(9)$ & $76.57(17)$ & $33.99(9)$ & $63.49(15)$ \\
\hline Benzimidazole (2) & & & $83.62(18)$ & $28.82(10)$ & $81.69(14)$ \\
\hline Hfa 1 & & & & $70.41(18)$ & $87.96(12)$ \\
\hline Hfa 2 & & & & & $58.15(17)$ \\
\hline
\end{tabular}

Table S33. Interplanar angles for $\left[\mathbf{L 6}\left(\mathrm{Lu}(\mathrm{hfa})_{3}\right)_{2}\right]$.

\begin{tabular}{|l|c|c|c|c|c|c|}
\hline Plane & $\mathrm{Bz}(1)^{a}$ & $\mathrm{Py}(1)^{b}$ & $\mathrm{Bz}(2)$ & $\mathrm{Bz}(3)$ & $\mathrm{Py}(2)$ & $\mathrm{Bz}(4)$ \\
\hline $\mathrm{Bz}(1)$ & & & & & & \\
\hline $\mathrm{Py}(1)$ & $4.37(18)$ & & & & & \\
\hline $\mathrm{Hfa}(1)$ & $84.0(2)$ & $81.6(3)$ & $60.8(2)$ & & & \\
\hline $\mathrm{Hfa}(2)$ & $32.01(19)$ & $55.0(3)$ & $21.29(18)$ & & & \\
\hline $\mathrm{Hfa}(3)$ & $61.3(2)$ & $64.8(2)$ & $71.06(16)$ & & & \\
\hline $\mathrm{Bz}(2)$ & $24.98(14)$ & $21.58(14)$ & & & & \\
\hline $\mathrm{Bz}(3)$ & & & $43.68(11)$ & & & \\
\hline $\mathrm{Py}(2)$ & & & & $31.24(14)$ & & \\
\hline $\mathrm{Hfa}(4)^{c}$ & & & & $72.98(16)$ & $63.11(19)$ & $76.23(19)$ \\
\hline $\mathrm{Hfa}(5)$ & & & & $22.50(14)$ & $36.48(16)$ & $43.79(13)$ \\
\hline $\mathrm{Hfa}(6)$ & & & & $87.4(2)$ & $58.0(2)$ & $56.9(2)$ \\
\hline $\mathrm{Bz}(4)$ & & & & $30.78(10)$ & $13.12(16)$ & \\
\hline
\end{tabular}


Table S34. Interplanar angles for [ $\left.\mathbf{L 6}\left(\mathrm{Yb}(\mathrm{hfa})_{3}\right)_{2}\right]$.

\begin{tabular}{|l|c|c|c|c|c|c|}
\hline Plane & $\mathrm{Bz}(1)^{a}$ & $\mathrm{Py}(1)^{b}$ & $\mathrm{Bz}(2)$ & $\mathrm{Bz}(3)$ & $\mathrm{Py}(2)$ & $\mathrm{Bz}(4)$ \\
\hline $\mathrm{Bz}(1)$ & & & & & & \\
\hline $\mathrm{Py}(1)$ & $1.8(2)$ & & & & & \\
\hline $\mathrm{Hfa}(1)$ & $79.3(3)$ & $77.9(3)$ & $57.5(3)$ & & & \\
\hline $\mathrm{Hfa}(2)$ & $33.5(3)$ & $33.0(2)$ & $23.9(2)$ & & & \\
\hline $\mathrm{Hfa}(3)$ & $62.4(3)$ & $63.5(2)$ & $70.0(2)$ & & & \\
\hline $\mathrm{Bz}(2)$ & $22.7(2)$ & $21.0(2)$ & & & & \\
\hline $\mathrm{Bz}(3)$ & & & $45.85(15)$ & & & \\
\hline $\mathrm{Py}(2)$ & & & & $32.38(17)$ & & \\
\hline $\mathrm{Hfa}(4)^{c}$ & & & & $90.1(4)$ & $59.1(3)$ & $58.5(3)$ \\
\hline $\mathrm{Hfa}(5)$ & & & & $72.4(2)$ & $63.1(2)$ & $76.3(3)$ \\
\hline $\mathrm{Hfa}(6)$ & & & & $21.6(2)$ & $34.7(2)$ & $42.30(2)$ \\
\hline $\mathrm{Bz}(4)$ & & & & $32.09(14)$ & $13.3(2)$ & \\
\hline
\end{tabular}


Table S35 Summary of crystal data, intensity measurements and structure refinements for $\left[\mathbf{L} 7\left(\mathrm{Eu}(\mathrm{hfa})_{3}\right)_{3}\right]$.

\begin{tabular}{|c|c|}
\hline Compound & {$\left[\mathbf{L} 7\left(\mathrm{Eu}(\mathrm{hfa})_{3}\right)_{3}\right]$} \\
\hline Empirical formula & $\mathrm{C}_{150} \mathrm{H}_{22} \mathrm{~F}_{54} \mathrm{O}_{21} \mathrm{Eu}_{3}$ \\
\hline Formula weight & 3970.64 \\
\hline Temperature & $120.00(14) \mathrm{K}$ \\
\hline Wavelength & $1.54184 \AA$ \\
\hline Crystal System, Space group & Monoclinic, $\mathrm{P} 21 / \mathrm{c}$ \\
\hline Unit cell dimensions & $\begin{array}{l}a=39.2391(9) \AA \\
b=20.0147(6) \AA \\
c=25.9985(5) \AA \\
\alpha=90^{\circ} \\
\beta=94.415(2)^{\circ} . \\
\gamma=90^{\circ}\end{array}$ \\
\hline Volume in $\AA^{3}$ & $20357.6(9)$ \\
\hline Calculated density & $1.296 \mathrm{~g} / \mathrm{cm}^{3}$ \\
\hline$Z$ & 4 \\
\hline Absorption coefficient & $7.456 \mathrm{~mm}^{-1}$ \\
\hline$F(000)$ & 7952 \\
\hline Theta range for data collection & 4.96 to $100.866^{\circ}$ \\
\hline Limiting indices & $-38 \leq h \leq 39,-17 \leq k \leq 20,-25 \leq l \leq 25$ \\
\hline Reflections collected / unique & $\begin{array}{l}64360 / 20803\left[R_{\mathrm{int}}=0.1411, \mathrm{R}_{\text {sigma }}\right. \\
=0.2059]\end{array}$ \\
\hline Data / restraints / parameters & $20803 / 1533 / 2437$ \\
\hline Goodness-of-fit on $F^{2}$ & 0.985 \\
\hline Final $R$ indexes $[I>2 \sigma(I)]$ & $\begin{array}{l}R_{1}=0.0812 \\
\mathrm{w} R_{2}=0.2126\end{array}$ \\
\hline$R$ indices (all data) & $R_{1}=0.1480, \mathrm{w} R_{2}=0.2382$ \\
\hline Largest diff. peak and hole & 0.92 and -0.67 e. $\AA^{-3}$ \\
\hline
\end{tabular}

Data were collected on the Rigaku XtaLAB synergy S equipped with a hybrid pixel hypix arc 150 detector. The crystal suffers from serious flaws: the diffraction is weak at high angle and a small second domain was attached to it (Figure S8). We were not able to find a better crystal so that we had to use this dataset. The best data integration was obtained using $\mathrm{XDS}^{\mathrm{S} 34-1}$. Data were solved with ShelXT ${ }^{\mathrm{S} 34-3}$ and redefined using SHELXL ${ }^{\mathrm{S} 34-4}$. Details of the structure refinement can be found in Table S34. Due to the low diffraction at high angle, the data were cut at $1 \AA \AA$ resolution. The side alkyl 
chains of the $\mathbf{L} 7$ ligands were heavily disordered and the model for them was highly approximative. Geometrical restraints were used as well as restrains on displacement parameters. The hexafluoroacetylacetonate (hfa) ligands were also very disordered. At one extremity of the complex, we were able to identify two alternative positions for these ligands that were modelled using four ligands. It was not impossible that such disorder is present to a lesser extent elsewhere. The squeeze/bypass ${ }^{\mathrm{S} 34-4}$ method implemented in olex $2^{\mathrm{S} 34-5}$ was used to take care of disordered solvent parts. The main hole of about 4400 cubic $\AA$ was found containing about 1070 electrons. This could be any of the solvent used for crystallization. The $R 1$ factor before squeeze was 0.13 and after squeeze 0.08 , and $\mathrm{w} R 2$ before squeeze was 0.36 and after squeeze was 0.24 .

\section{References}

S34-1 Kabsch, W., Xds. Acta Cryst. D 2010, 66, 125-132.

S34-2 Sheldrick, G. M., Crystal Structure Refinement with SHELXL. Acta Cryst. C 2015, 71, 3-8.

S34-3 Sheldrick, G. M., Acta Cryst. A 2015. 71, 3-8

S34-4 Spek, A. L., PLATON SQUEEZE: A Tool for the Calculation of the Disordered Solvent Contribution to the Calculated Structure Factors. Acta Cryst. C 2015, 71, 9-18.

S34-5 Dolomanov, O. V.; Bourhis, L. J.; Gildea, R. J.; Howard, J. A. K.; Puschmann, H., OLEX2: A Complete Structure Solution, Refinement and Analysis Program. J. Appl. Crystallogr. 2009, 42, 339-341.

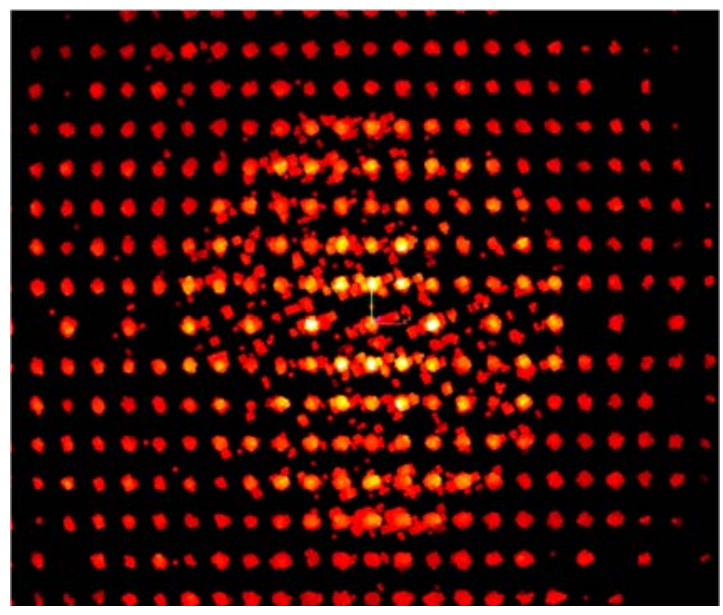

Figure S8. Reconstruction of the reciprocal space, view along c*. There are extra reflections that belongs to a second weaker domain. 
a)

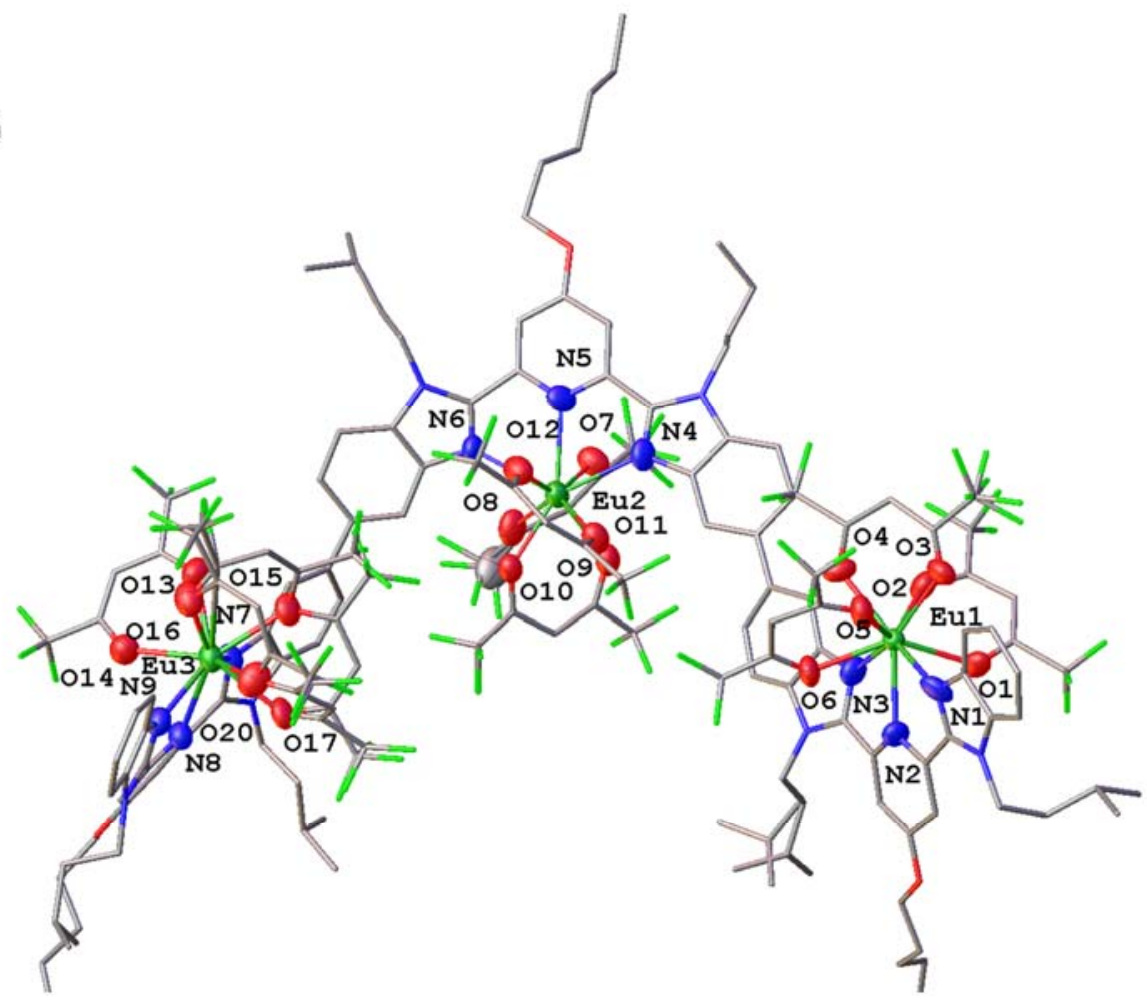

b)

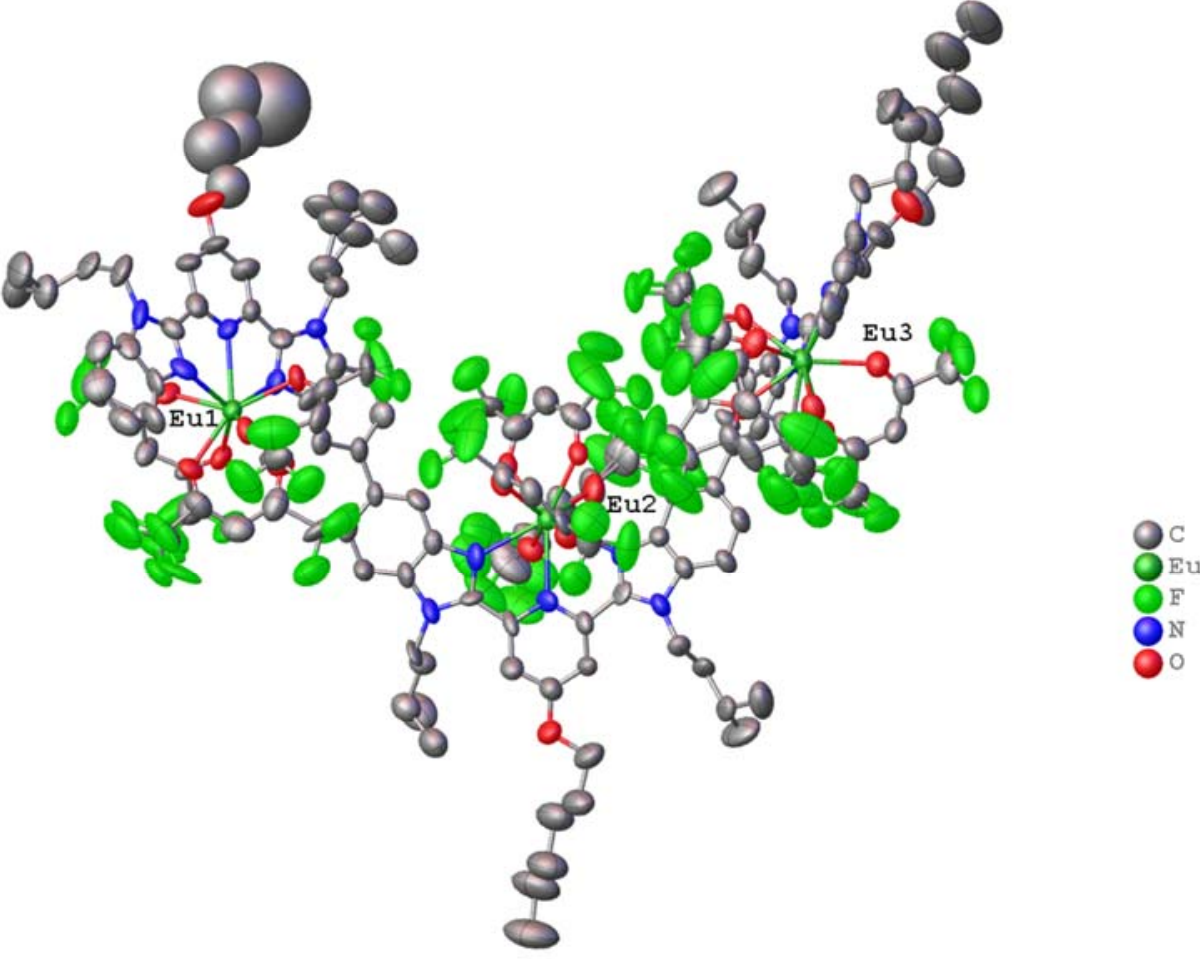

Figure S9. a) A low-resolution molecular structure of complex $\left[\mathbf{L 7}\left(\mathrm{Eu}(\mathrm{hfa})_{3}\right)_{3}\right]$ with numbering scheme (data were cut at $1 \AA$ and disorder is omitted for clarity). b) View of the asymmetric unit with displacement parameters at 50\% probability level. 
Table S36. Selected bond distances $(\AA)$, in [L7(Eu(hfa $\left.\left.)_{3}\right)_{3}\right]$

\begin{tabular}{|ll|ll|ll|}
\hline Atoms 1-2 & $(\AA)$ & Atoms 1-2 & $(\AA)$ & Atoms 1-2 & $(\AA)$ \\
\hline $\mathrm{Eu}(1)-\mathrm{N}(1)$ & 2.53 & $\mathrm{Eu}(2)-\mathrm{N}(5)$ & 2.60 & $\mathrm{Eu}(3)-\mathrm{N}(9)$ & 2.56 \\
$\mathrm{Eu}(1)-\mathrm{N}(2)$ & 2.57 & $\mathrm{Eu}(2)-\mathrm{N}(6)$ & 2.55 & $\mathrm{Eu}(3)-\mathrm{N}(8)$ & 2.61 \\
$\mathrm{Eu}(1)-\mathrm{N}(3)$ & 2.52 & $\mathrm{Eu}(2)-\mathrm{N}(4)$ & 2.54 & $\mathrm{Eu}(3)-\mathrm{N}(7)$ & 2.50 \\
$\mathrm{Eu}(1)-\mathrm{O}(1)$ & 2.39 & $\mathrm{Eu}(2)-\mathrm{O}(7)$ & 2.42 & $\mathrm{Eu}(3)-\mathrm{O}(20)$ & 2.42 \\
$\mathrm{Eu}(1)-\mathrm{O}(2)$ & 2.48 & $\mathrm{Eu}(2)-\mathrm{O}(8)$ & 2.48 & $\mathrm{Eu}(3)-\mathrm{O}(16)$ & 2.41 \\
$\mathrm{Eu}(1)-\mathrm{O}(3)$ & 2.37 & $\mathrm{Eu}(2)-\mathrm{O}(9)$ & 2.39 & $\mathrm{Eu}(3)-\mathrm{O}(14)$ & 2.38 \\
$\mathrm{Eu}(1)-\mathrm{O}(4)$ & 2.35 & $\mathrm{Eu}(2)-\mathrm{O}(10)$ & 2.39 & $\mathrm{Eu}(3)-\mathrm{O}(13)$ & 2.46 \\
$\mathrm{Eu}(1)-\mathrm{O}(5)$ & 2.48 & $\mathrm{Eu}(2)-\mathrm{O}(11)$ & 2.46 & $\mathrm{Eu}(3)-\mathrm{O}(17)$ & 2.44 \\
$\mathrm{Eu}(1)-\mathrm{O}(6)$ & 2.40 & $\mathrm{Eu}(2)-\mathrm{O}(12)$ & 2.41 & $\mathrm{Eu}(3)-\mathrm{O}(15)$ & 2.39 \\
\hline
\end{tabular}

Table S37. Chelate angles $\left(^{\circ}\right)$ in $\left[\mathbf{L} 7\left(\mathrm{Eu}(\mathrm{hfa})_{3}\right)_{3}\right]$.

\begin{tabular}{|ll|ll|ll|}
\hline Atoms 1-2-3 & $\left(^{\circ}\right)$ & Atoms 1-2-3 & $\left(^{\circ}\right)$ & Atoms 1-2-3 & $\left(^{\circ}\right)$ \\
\hline $\mathrm{N}(1)-\mathrm{Eu}(1)-\mathrm{N}(2)$ & 64.37 & $\mathrm{~N}(4)-\mathrm{Eu}(2)-\mathrm{N}(5)$ & 62.15 & $\mathrm{~N}(7)-\mathrm{Eu}(3)-\mathrm{N}(8)$ & 63.87 \\
$\mathrm{~N}(1)-\mathrm{Eu}(1)-\mathrm{N}(3)$ & 128.44 & $\mathrm{~N}(4)-\mathrm{Eu}(2)-\mathrm{N}(6)$ & 126.33 & $\mathrm{~N}(7)-\mathrm{Eu}(3)-\mathrm{N}(9)$ & 127.01 \\
$\mathrm{~N}(2)-\mathrm{Eu}(1)-\mathrm{N}(3)$ & 64.08 & $\mathrm{~N}(5)-\mathrm{Eu}(2)-\mathrm{N}(6)$ & 64.19 & $\mathrm{~N}(8)-\mathrm{Eu}(3)-\mathrm{N}(9)$ & 63.30 \\
$\mathrm{O}(1)-\mathrm{Eu}(1)-\mathrm{O}(2)$ & 68.78 & $\mathrm{O}(7)-\mathrm{Eu}(2)-\mathrm{O}(8)$ & 68.72 & $\mathrm{O}(13)-\mathrm{Eu}(3)-\mathrm{O}(14)$ & 70.00 \\
$\mathrm{O}(3)-\mathrm{Eu}(1)-\mathrm{O}(4)$ & 76.52 & $\mathrm{O}(9)-\mathrm{Eu}(2)-\mathrm{O}(10)$ & 76.03 & $\mathrm{O}(16)-\mathrm{Eu}(3)-\mathrm{O}(20)$ & 69.46 \\
$\mathrm{O}(3)-\mathrm{Eu}(1)-\mathrm{O}(4)$ & 69.86 & $\mathrm{O}(11)-\mathrm{Eu}(2)-\mathrm{O}(12)$ & 68.05 & $\mathrm{O}(15)-\mathrm{Eu}(3)-\mathrm{O}(17)$ & 72.06 \\
\hline
\end{tabular}


Table S38. Selected bond angles $\left(^{\circ}\right)$ in $\left[\mathbf{L 7}\left(\mathrm{Eu}(\mathrm{hfa})_{3}\right)_{3}\right]$.

\begin{tabular}{|lc|lc|lc|}
\hline Atoms 1-2-3 & $\left({ }^{\circ}\right)$ & \multicolumn{1}{|c|}{ Atoms 1-2 } & $\left(^{\circ}\right)$ & \multicolumn{1}{|c|}{ Atoms 1-2 } & $\left(^{\circ}\right)$ \\
\hline $\mathrm{O}(1)-\mathrm{Eu}(1)-\mathrm{N}(1)$ & 71.16 & $\mathrm{O}(7)-\mathrm{Eu}(2)-\mathrm{N}(4)$ & 69.81 & $\mathrm{O}(13)-\mathrm{Eu}(3)-\mathrm{N}(7)$ & 72.58 \\
$\mathrm{O}(1)-\mathrm{Eu}(1)-\mathrm{N}(2)$ & 70.13 & $\mathrm{O}(7)-\mathrm{Eu}(2)-\mathrm{N}(5)$ & 69.39 & $\mathrm{O}(13)-\mathrm{Eu}(3)-\mathrm{N}(8)$ & 121.35 \\
$\mathrm{O}(1)-\mathrm{Eu}(1)-\mathrm{N}(3)$ & 91.03 & $\mathrm{O}(7)-\mathrm{Eu}(2)-\mathrm{N}(6)$ & 92.40 & $\mathrm{O}(13)-\mathrm{Eu}(3)-\mathrm{N}(9)$ & 135.77 \\
$\mathrm{O}(2)-\mathrm{Eu}(1)-\mathrm{N}(1)$ & 135.09 & $\mathrm{O}(8)-\mathrm{Eu}(2)-\mathrm{N}(4)$ & 136.11 & $\mathrm{O}(14)-\mathrm{Eu}(3)-\mathrm{N}(7)$ & 84.03 \\
$\mathrm{O}(2)-\mathrm{Eu}(1)-\mathrm{N}(2)$ & 117.63 & $\mathrm{O}(8)-\mathrm{Eu}(2)-\mathrm{N}(5)$ & 114.09 & $\mathrm{O}(14)-\mathrm{Eu}(3)-\mathrm{N}(8)$ & 68.27 \\
$\mathrm{O}(2)-\mathrm{Eu}(1)-\mathrm{N}(3)$ & 71.94 & $\mathrm{O}(8)-\mathrm{Eu}(2)-\mathrm{N}(6)$ & 69.31 & $\mathrm{O}(14)-\mathrm{Eu}(3)-\mathrm{N}(9)$ & 73.31 \\
$\mathrm{O}(3)-\mathrm{Eu}(1)-\mathrm{N}(1)$ & 63.86 & $\mathrm{O}(9)-\mathrm{Eu}(2)-\mathrm{N}(4)$ & 83.74 & $\mathrm{O}(15)-\mathrm{Eu}(3)-\mathrm{N}(7)$ & 84.15 \\
$\mathrm{O}(3)-\mathrm{Eu}(1)-\mathrm{N}(2)$ & 143.35 & $\mathrm{O}(9)-\mathrm{Eu}(2)-\mathrm{N}(5)$ & 136.34 & $\mathrm{O}(15)-\mathrm{Eu}(3)-\mathrm{N}(8)$ & 136.34 \\
$\mathrm{O}(3)-\mathrm{Eu}(1)-\mathrm{N}(3)$ & 71.94 & $\mathrm{O}(9)-\mathrm{Eu}(2)-\mathrm{N}(6)$ & 140.83 & $\mathrm{O}(15)-\mathrm{Eu}(3)-\mathrm{N}(9)$ & 140.92 \\
$\mathrm{O}(4)-\mathrm{Eu}(1)-\mathrm{N}(1)$ & 140.44 & $\mathrm{O}(10)-\mathrm{Eu}(2)-\mathrm{N}(4)$ & 139.59 & $\mathrm{O}(16)-\mathrm{Eu}(3)-\mathrm{N}(7)$ & 140.15 \\
$\mathrm{O}(4)-\mathrm{Eu}(1)-\mathrm{N}(2)$ & 141.03 & $\mathrm{O}(10)-\mathrm{Eu}(2)-\mathrm{N}(5)$ & 147.58 & $\mathrm{O}(16)-\mathrm{Eu}(3)-\mathrm{N}(8)$ & 144.50 \\
$\mathrm{O}(4)-\mathrm{Eu}(1)-\mathrm{N}(3)$ & 84.85 & $\mathrm{O}(10)-\mathrm{Eu}(2)-\mathrm{N}(6)$ & 89.41 & $\mathrm{O}(16)-\mathrm{Eu}(3)-\mathrm{N}(9)$ & 86.30 \\
$\mathrm{O}(5)-\mathrm{Eu}(1)-\mathrm{N}(1)$ & 69.92 & $\mathrm{O}(11)-\mathrm{Eu}(2)-\mathrm{N}(4)$ & 72.01 & $\mathrm{O}(17)-\mathrm{Eu}(3)-\mathrm{N}(7)$ & 70.85 \\
$\mathrm{O}(5)-\mathrm{Eu}(1)-\mathrm{N}(2)$ & 114.85 & $\mathrm{O}(11)-\mathrm{Eu}(2)-\mathrm{N}(5)$ & 115.52 & $\mathrm{O}(17)-\mathrm{Eu}(3)-\mathrm{N}(8)$ & 68.82 \\
$\mathrm{O}(5)-\mathrm{Eu}(1)-\mathrm{N}(3)$ & 135.58 & $\mathrm{O}(11)-\mathrm{Eu}(2)-\mathrm{N}(6)$ & 134.30 & $\mathrm{O}(17)-\mathrm{Eu}(3)-\mathrm{N}(9)$ & 94.67 \\
$\mathrm{O}(6)-\mathrm{Eu}(1)-\mathrm{N}(1)$ & 96.27 & $\mathrm{O}(12)-\mathrm{Eu}(2)-\mathrm{N}(4)$ & 95.47 & $\mathrm{O}(20)-\mathrm{Eu}(3)-\mathrm{N}(7)$ & 137.12 \\
$\mathrm{O}(6)-\mathrm{Eu}(1)-\mathrm{N}(2)$ & 71.79 & $\mathrm{O}(12)-\mathrm{Eu}(2)-\mathrm{N}(5)$ & 73.73 & $\mathrm{O}(20)-\mathrm{Eu}(3)-\mathrm{N}(8)$ & 112.14 \\
$\mathrm{O}(6)-\mathrm{Eu}(1)-\mathrm{N}(3)$ & 68.18 & $\mathrm{O}(12)-\mathrm{Eu}(2)-\mathrm{N}(6)$ & 68.67 & $\mathrm{O}(20)-\mathrm{Eu}(3)-\mathrm{N}(9)$ & 69.66 \\
\hline
\end{tabular}


Table S39. Selected least-squares planes data of complex $\left[\mathbf{L 7}\left(\mathrm{Eu}(\mathrm{hfa})_{3}\right)_{3}\right]$.

\begin{tabular}{|c|c|c|c|}
\hline Plane & Abbreviation & $\begin{array}{c}\text { Max. } \\
\text { deviation }(\AA)\end{array}$ & Atom \\
\hline $\begin{array}{l}\text { Benzimidazole } 1 \\
\mathrm{C}(1) \mathrm{C}(2) \mathrm{C}(3) \mathrm{C}(4) \mathrm{C}(5) \mathrm{C}(6) \mathrm{C}(7) \mathrm{N}(1) \mathrm{N}(10)\end{array}$ & Bz1 & 0.041 & $\mathrm{~N}(11)$ \\
\hline $\begin{array}{l}\text { Pyridine } 1 \\
\mathrm{~N}(2) \mathrm{C}(13) \mathrm{C}(14) \mathrm{C}(15) \mathrm{C}(16) \mathrm{C}(17)\end{array}$ & Pyl & -0.041 & $C(14)$ \\
\hline $\begin{array}{l}\text { Benzimidazole } 2 \\
C(25) C(30) C(27) C(29) C(26) C(28) C(106) N(11) N(3)\end{array}$ & $\mathrm{Bz} 2$ & 0.050 & $\mathrm{O}(29)$ \\
\hline $\begin{array}{l}\text { Benzimidazole } 3 \\
\mathrm{C}(38) \mathrm{C}(41) \mathrm{C}(42) \mathrm{C}(36) \mathrm{C}(37) \mathrm{C}(39) \mathrm{C}(40) \mathrm{N}(12) \mathrm{N}(4)\end{array}$ & $\mathrm{Bz} 3$ & -0.007 & $\mathrm{C}(41)$ \\
\hline $\begin{array}{l}\text { Pyridine } 1 \\
N(5) C(48) C(49) C(50) C(51) C(52)\end{array}$ & Py2 & 0.040 & $C(52)$ \\
\hline $\begin{array}{l}\text { Benzimidazole } 4 \\
C(59) \mathrm{C}(60) \mathrm{C}(61) \mathrm{C}(62) \mathrm{C}(63) \mathrm{C}(64) \mathrm{C}(65) \mathrm{N}(13) \mathrm{N}(6)\end{array}$ & $\mathrm{Bz} 4$ & -0.040 & $\mathrm{~N}(6)$ \\
\hline $\begin{array}{l}\text { Benzimidazole } 5 \\
C(71) C(72) C(73) C(74) C(75) C(76) C(77) N(14) N(7)\end{array}$ & Bz5 & -0.040 & $\mathrm{~N}(7)$ \\
\hline $\begin{array}{l}\text { Pyridine } 1 \\
\mathrm{~N}(8) \mathrm{C}(83) \mathrm{C}(84) \mathrm{C}(85) \mathrm{C}(86) \mathrm{C}(87)\end{array}$ & Py3 & 0.017 & $C(84)$ \\
\hline $\begin{array}{l}\text { Benzimidazole } 6 \\
C(95) \mathrm{C}(96) \mathrm{C}(97) \mathrm{C}(98) \mathrm{C}(99) \mathrm{C}(100) \mathrm{C}(94) \mathrm{N}(9) \mathrm{N}(15)\end{array}$ & Bz6 & -0.022 & C(99) \\
\hline $\begin{array}{l}\text { Hexafluoroacetylacetonate1 } \\
\mathrm{C}(120) \mathrm{C}(121) \mathrm{C}(122) \mathrm{O}(1) \mathrm{O}(2)\end{array}$ & Hfa1 & -0.034 & $C(122)$ \\
\hline $\begin{array}{l}\text { Hexafluoroacetylacetonate } 2 \\
\mathrm{C}(125) \mathrm{C}(126) \mathrm{C}(127) \mathrm{O}(3) \mathrm{O}(4)\end{array}$ & Hfa2 & 0.04 & $C(125)$ \\
\hline $\begin{array}{l}\text { Hexafluoroacetylacetonate } 3 \\
\mathrm{C}(131) \mathrm{C}(132) \mathrm{C}(133) \mathrm{O}(5) \mathrm{O}(6)\end{array}$ & Hfa3 & -0.04 & $C(130)$ \\
\hline $\begin{array}{l}\text { Hexafluoroacetylacetonate } 4 \\
\mathrm{C}(135) \mathrm{C}(136) \mathrm{C}(137) \mathrm{O}(7) \mathrm{O}(8)\end{array}$ & Hfa4 & -0.046 & $\mathrm{O}(8)$ \\
\hline $\begin{array}{l}\text { Hexafluoroacetylacetonate } 5 \\
\mathrm{C}(140) \mathrm{C}(141) \mathrm{C}(142) \mathrm{O}(9) \mathrm{O}(10)\end{array}$ & Hfa5 & 0.041 & $\mathrm{C}(140)$ \\
\hline $\begin{array}{l}\text { Hexafluoroacetylacetonate } 6 \\
\mathrm{C}(145) \mathrm{C}(146) \mathrm{C}(147) \mathrm{O}(11) \mathrm{O}(12)\end{array}$ & Hfa6 & 0.024 & $C(147)$ \\
\hline $\begin{array}{l}\text { Hexafluoroacetylacetonate } 7 \\
\mathrm{C}(150) \mathrm{C}(151) \mathrm{C}(152) \mathrm{O}(13) \mathrm{O}(14)\end{array}$ & $\mathrm{Hfa} 7$ & 0.036 & $C(150)$ \\
\hline $\begin{array}{l}\text { Hexafluoroacetylacetonate } 8^{a} \\
\mathrm{C}(170) \mathrm{C}(171) \mathrm{C}(172) \mathrm{O}(16) \mathrm{O}(20)\end{array}$ & Hfa8 & -0.033 & $C(170)$ \\
\hline $\begin{array}{l}\text { Hexafluoroacetylacetonate } 9^{a} \\
\mathrm{C}(160) \mathrm{C}(161) \mathrm{C}(162) \mathrm{O}(15) \mathrm{O}(17)\end{array}$ & Hfa9 & -0.013 & $C(160)$ \\
\hline
\end{tabular}

${ }^{a}$ Disorder in the structure. 
Table S40. Interplanar angles for $\left[\mathbf{L 7}\left(\mathrm{Eu}(\mathrm{hfa})_{3}\right)_{3}\right]$.

\begin{tabular}{|c|c|c|c|c|c|c|c|c|c|}
\hline \multicolumn{10}{|c|}{ Interplanar angles $\left[\mathbf{L} 7\left(\mathrm{Eu}(\mathrm{hfa})_{3}\right)_{3}\right]\left(^{\circ}\right)$} \\
\hline Plane & $\mathrm{Bz}(1)^{a}$ & $\operatorname{Py}(1)^{b}$ & $\mathrm{Bz}(2)$ & $\mathrm{Bz}(3)$ & Py(2) & $\mathrm{Bz}(4)$ & $\mathrm{Bz}(5)$ & $\operatorname{Py}(3)$ & $\mathrm{Bz}(6)$ \\
\hline Py(1) & $2.3(4)$ & & 21.1(4) & & & & & & \\
\hline $\operatorname{Hfa}(1)$ & $76.9(4)$ & $74.8(6)$ & $54.7(4)$ & & & & & & \\
\hline $\operatorname{Hfa}(2)^{c}$ & $31.6(3)$ & $30.2(5)$ & $22.4(3)$ & & & & & & \\
\hline $\mathrm{Bz}(2)$ & $23.3(3)$ & $21.1(4)$ & & $36.4(3)$ & & & & & \\
\hline $\mathrm{Bz}(3)$ & & & $36.4(3)$ & & $2.7(4)$ & $24.8(3)$ & & & \\
\hline $\operatorname{Py}(2)$ & & & & $2.7(4)$ & & $22.9(4)$ & & & \\
\hline $\mathrm{Hfa}(4)$ & & & & $83.9(6)$ & $81.9(6)$ & $59.2(5)$ & & & \\
\hline $\mathrm{Bz}(5)$ & & & & & & $44.0(3)$ & & $29.2(4)$ & $25.3(3)$ \\
\hline $\operatorname{Py}(3)$ & & & & & & & $29.2(4)$ & & $20.6(4)$ \\
\hline $\mathrm{Hfa}(7)$ & & & & & & & $91.7(7)$ & $63.4(6)$ & $70.3(4)$ \\
\hline $\mathrm{Hfa}(8)$ & & & & & & & $94.7(5)$ & $81.5(5)$ & $70.6(5)$ \\
\hline $\mathrm{Hfa}(9)$ & & & & & & & $53.6(5)$ & $79.0(5)$ & $78.5(5)$ \\
\hline $\mathrm{Bz}(6)$ & & & & & & & $25.3(3)$ & $20.6(4)$ & \\
\hline
\end{tabular}

${ }^{a}$ Benzimidazole $1,{ }^{b}$ Pyridine $1{ }^{c}$ Hexafluoroacetylacetonate 
Table S41 Average Bond Lengths $(d)$, Bond Valences $\left(\bar{\nu}_{\mathrm{Ln}-\mathrm{N}}\right.$ and $\left.\bar{v}_{\mathrm{Ln}-\mathrm{O}}\right)$ and Bond Valence Sums $\left(V_{\mathrm{Ln}, j}\right)$ in the Crystal Structures of $\left[\mathbf{L} 3 \mathrm{Ln}(\mathrm{hfa})_{3}\right](\operatorname{Ln}=$ $\mathrm{La}, \mathrm{Lu})$ and $\left[\mathbf{L 6}\left(\mathrm{Ln}(\mathrm{hfa})_{3}\right)_{2}\right](\mathrm{Ln}=\mathrm{La}, \mathrm{Yb}, \mathrm{Lu})$

\begin{tabular}{|c|c|c|c|c|c|c|c|c|}
\hline Complex & $\begin{array}{c}d \mathrm{Ln}-\mathrm{N}(\mathrm{py}) \\
/ \AA\end{array}$ & $\overline{\bar{v}_{\mathrm{Ln}-\mathrm{N}(\mathrm{py})}}$ a & $\begin{array}{c}d \mathrm{Ln}-\mathrm{N}(\mathrm{bzim}) \\
/ \AA^{b}\end{array}$ & $\overline{\bar{v}}_{\mathrm{Ln}-\mathrm{N}(\text { bzim })} a, b$ & $\bar{\nu}_{\mathrm{Ln}-\mathrm{N}} a, b$ & $\begin{array}{c}d \mathrm{Ln}-\mathrm{O}(\mathrm{hfa}) \\
/ \AA^{b}\end{array}$ & $\overline{\bar{\nu}}_{\mathrm{Ln}-\mathrm{O}(\mathrm{hfac})} a, b$ & $V_{\mathrm{Ln}, j}^{c}$ \\
\hline$\left[\mathbf{L 3 L a}(\mathrm{hfa})_{3}\right]$ & 2.766 & 0.255 & $2.64(3)$ & $0.36(3)$ & $0.32(6)$ & $2.52(3)$ & $0.36(3)$ & 3.155 \\
\hline$\left[\mathbf{L} 3 \mathrm{Lu}(\mathrm{hfa})_{3}\right]$ & 2.532 & 0.269 & $2.462(8)$ & $0.325(7)$ & $0.31(3)$ & $2.35(6)$ & $0.34(5)$ & 2.976 \\
\hline$\left[\mathbf{L 6}\left(\mathrm{La}(\mathrm{hfa})_{3}\right)_{2}\right]$ & 2.703 & 0.303 & $2.640(3)$ & $0.359(3)$ & $0.34(3)$ & $2.52(2)$ & $0.36(2)$ & 3.202 \\
\hline$\left[\mathbf{L 6}\left(\mathrm{Yb}(\mathrm{hfa})_{3}\right)_{2}\right]$ & $2.51(2)$ & $0.30(1)$ & $2.48(1)$ & $0.33(1)$ & $0.32(2)$ & $2.36(7)$ & $0.34(6)$ & $3.00(3)$ \\
\hline$\left[\mathbf{L 6}\left(\mathrm{Lu}(\mathrm{hfa})_{3}\right)_{2}\right]$ & $2.502(5)$ & $0.292(4)$ & $2.46(1)$ & $0.33(1)$ & $0.32(2)$ & $2.35(6)$ & $0.34(5)$ & $2.996(4)$ \\
\hline
\end{tabular}

$\bar{a} v_{\mathrm{Ln}, j}=\exp \left[\left(R_{\mathrm{Ln}, j}-d_{\mathrm{Ln}, j}\right) / b\right]$ where $d \mathrm{Ln}, j$ is the bond length, $R_{\mathrm{Ln}, j}$ corresponds to the bond valence parameters and $b=0.37 \AA$ is a universal scaling constant. ${ }^{28 b}$ Average of several values with the associated standard deviations given between brackets. ${ }^{c} V_{\mathrm{Ln}}=\sum_{j} v_{\mathrm{Ln}, j}$. 
Table S42 Dihedral angle $(\not)$ between the coordination units in $\left[\mathbf{L 6}\left(\operatorname{Ln}(\mathrm{hfa})_{3}\right)_{2}\right](\mathrm{Ln}=\mathrm{La}, \mathrm{Eu}, \mathrm{Yb}$, $\mathrm{Lu})$ and $\left[\mathbf{L 7}\left(\mathrm{Eu}(\mathrm{hfa})_{3}\right)_{3}\right]$.

\begin{tabular}{lcccc}
\hline Complexes & $\Phi_{1-2}$ & $\Phi_{2-3}$ & $\begin{array}{c}\text { Intermetallic distance } \\
(\AA) \\
\left(d_{1-2}\right)\end{array}$ & $\begin{array}{c}\text { Intermetallic distance } \\
(\AA)\end{array}$ \\
\hline $\mathbf{L 6}$ & 46.72 & - & - & $\left(d_{1-3}\right)$ \\
{$\left[\mathbf{L 6}\left(\mathrm{La}(\mathrm{hfa})_{3}\right)_{2}\right]$} & 0 & - & 11.88 & - \\
{$\left[\mathbf{L 6}\left(\mathrm{Eu}(\mathrm{hfa})_{3}\right)_{2}\right]$} & 0 & - & 11.88 & - \\
{$\left[\mathbf{L 6}\left(\mathrm{Yb}(\mathrm{hfa})_{3}\right)_{2}\right]$} & 45.66 & - & 10.58 & - \\
{$\left[\mathbf{L 6}\left(\mathrm{Lu}(\mathrm{hfa})_{3}\right)_{2}\right]$} & 43.49 & - & 10.58 & - \\
{$\left[\mathbf{L 7}\left(\mathrm{Eu}(\mathrm{hfa})_{3}\right)_{3}\right]$} & 36.4 & 44.0 & 10.89 & 18.08 \\
\hline
\end{tabular}


a) $\left[\mathbf{L B E u}(\mathrm{hfa})_{3}\right]$

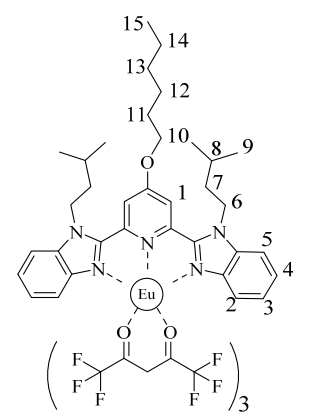

2
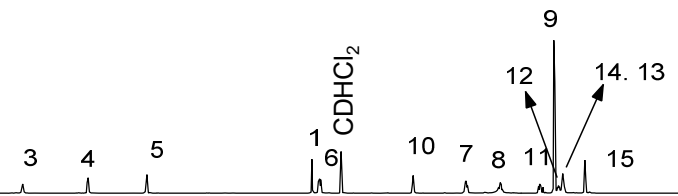

b) $\left[\mathrm{L4Eu}(\mathrm{hfa})_{3}\right]$

63

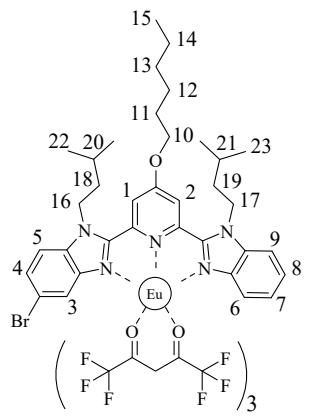

c) $\left[\mathbf{L 5 E u}(\mathrm{hfa})_{3}\right]$
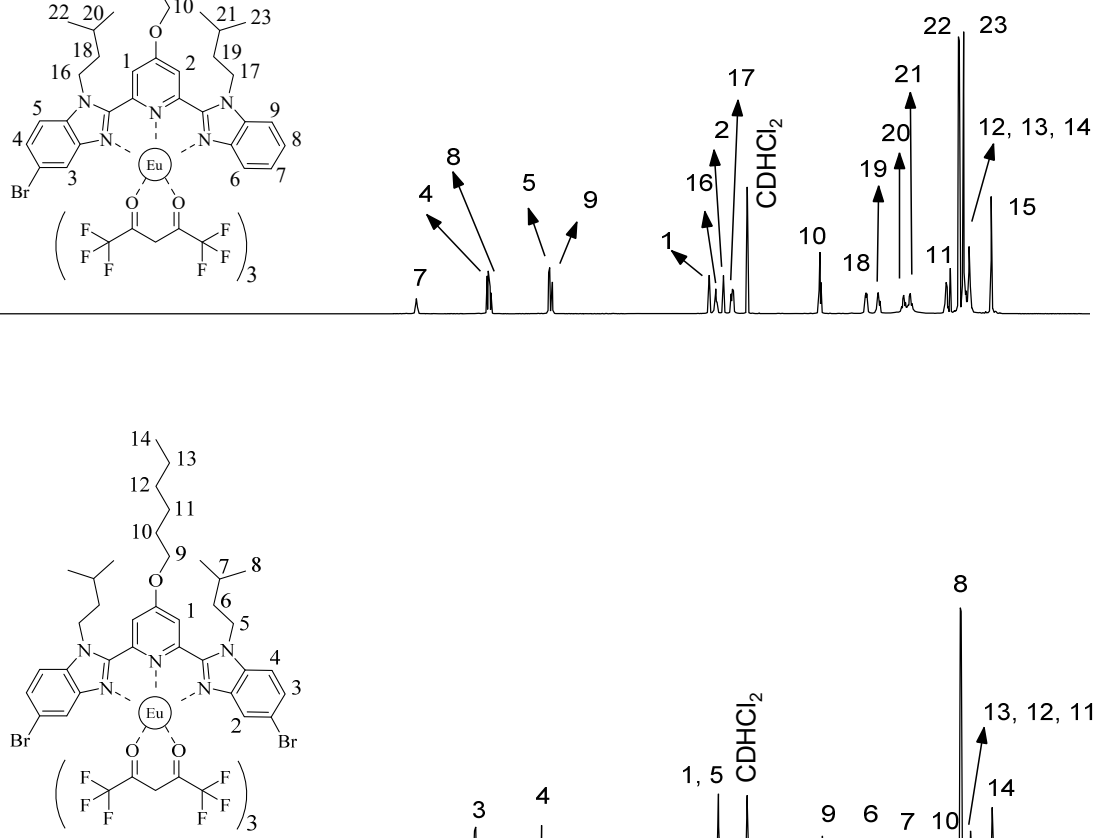

2

$\begin{array}{llllllll}25 & 24 & 23 & 22 & 21 & 20 & 19\end{array}$

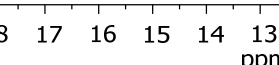

Figure S10. H NMR spectra with numbering scheme of a) $\left[\mathbf{L 3 E u}(\mathrm{hfa})_{3}\right]$, b) $\left[\mathbf{L} 4 \mathrm{Eu}(\mathrm{hfa})_{3}\right]$ and c) [L5Eu(hfa) $\left.)_{3}\right]$ complexes in $\mathrm{CD}_{2} \mathrm{Cl}_{2}$ at $298 \mathrm{~K}$. 
a) $\left[\mathbf{L 6}\left(\mathrm{Eu}(\mathrm{hfa})_{3}\right)_{2}\right]$
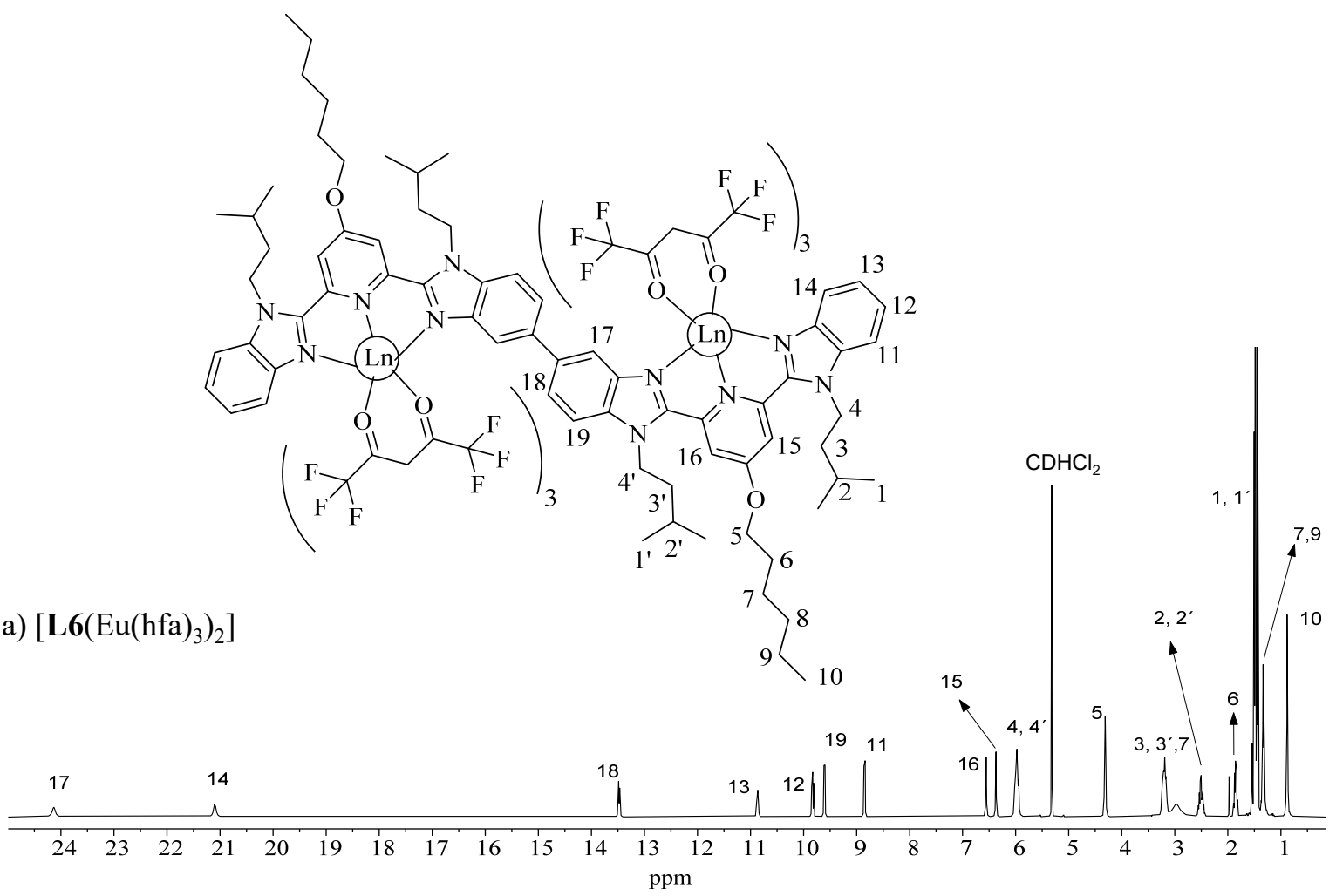

b) $\left[\mathbf{L 6}\left(\mathrm{La}(\mathrm{hfa})_{3}\right)_{2}\right]$

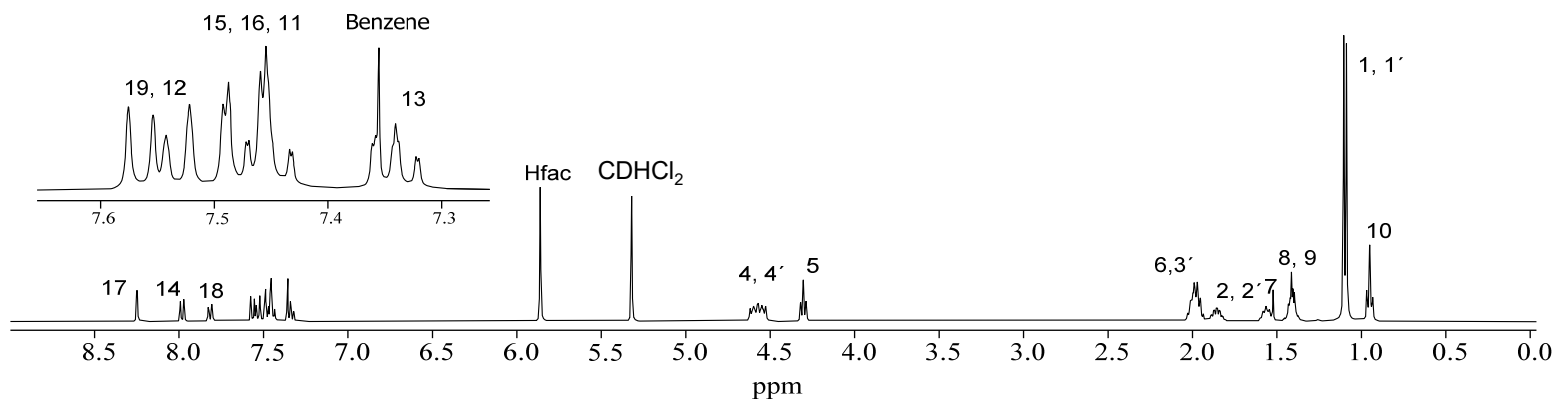

Figure S11. H NMR spectra with numbering scheme of a) $\left[\mathbf{L 6}\left(\mathrm{Eu}(\mathrm{hfa})_{3}\right)_{2}\right]$ and b) $\left[\mathbf{L 6}\left(\mathrm{La}(\mathrm{hfa})_{3}\right)_{2}\right]$ complexes in $\mathrm{CD}_{2} \mathrm{Cl}_{2}$ at $298 \mathrm{~K}$. 
a) $\left[\mathbf{L} 7\left(\mathrm{Eu}(\mathrm{hfa})_{3}\right)_{3}\right]$
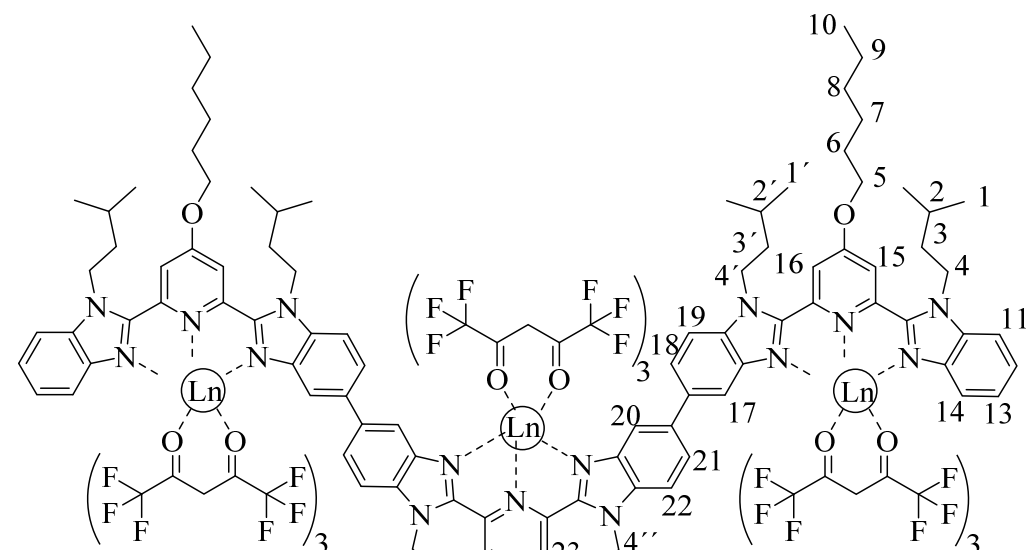

$\square$

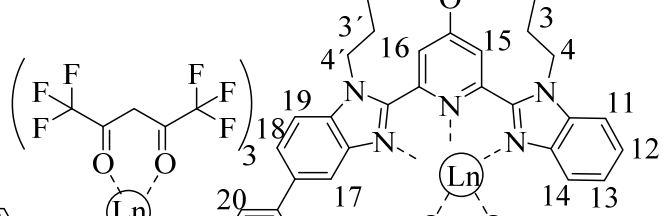

$\mathrm{CDHCl}_{2}$
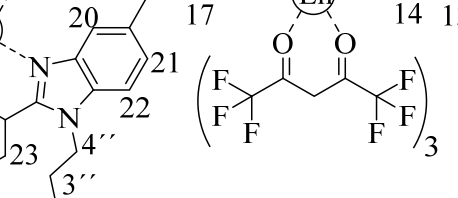

$\overbrace{5{ }^{\prime}}{ }^{3 \prime \prime} 1^{\prime \prime}$

$6^{\prime}$

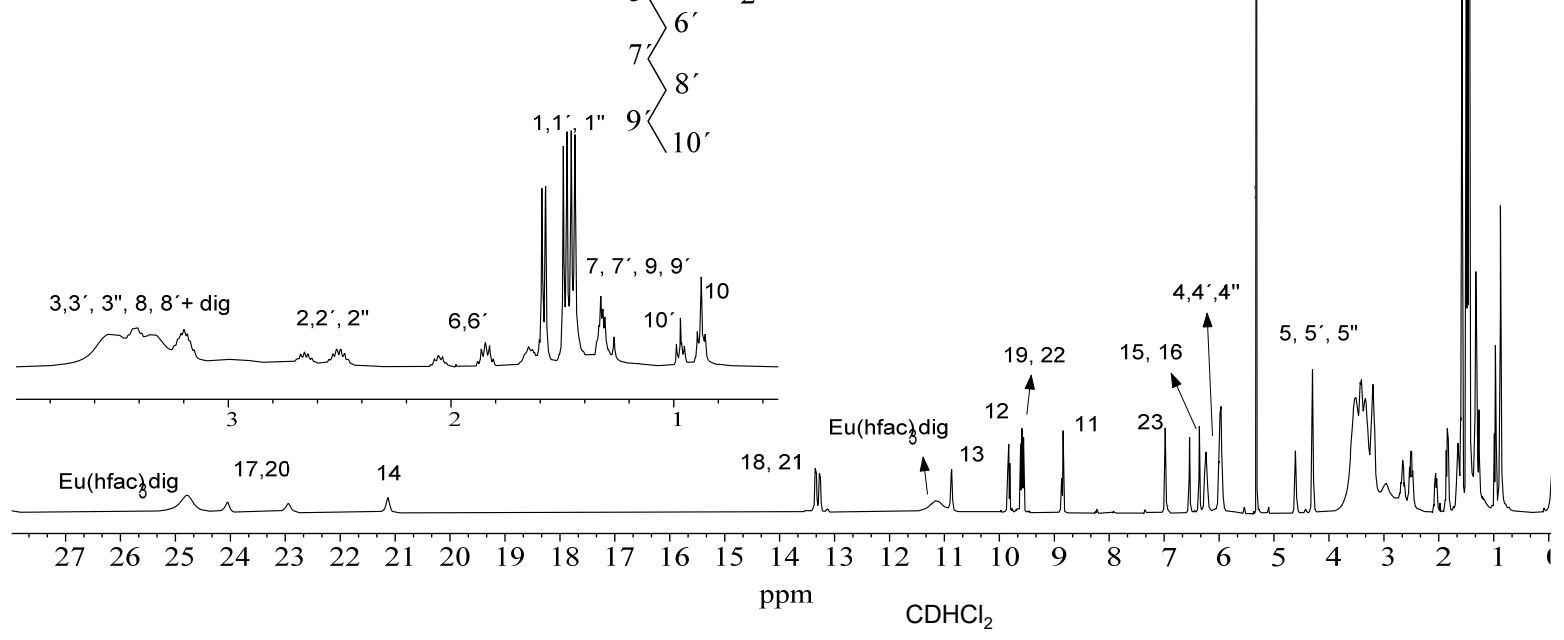

b) $\left[\mathbf{L} 7\left(\mathrm{La}(\mathrm{hfa})_{3}\right)_{3}\right]$

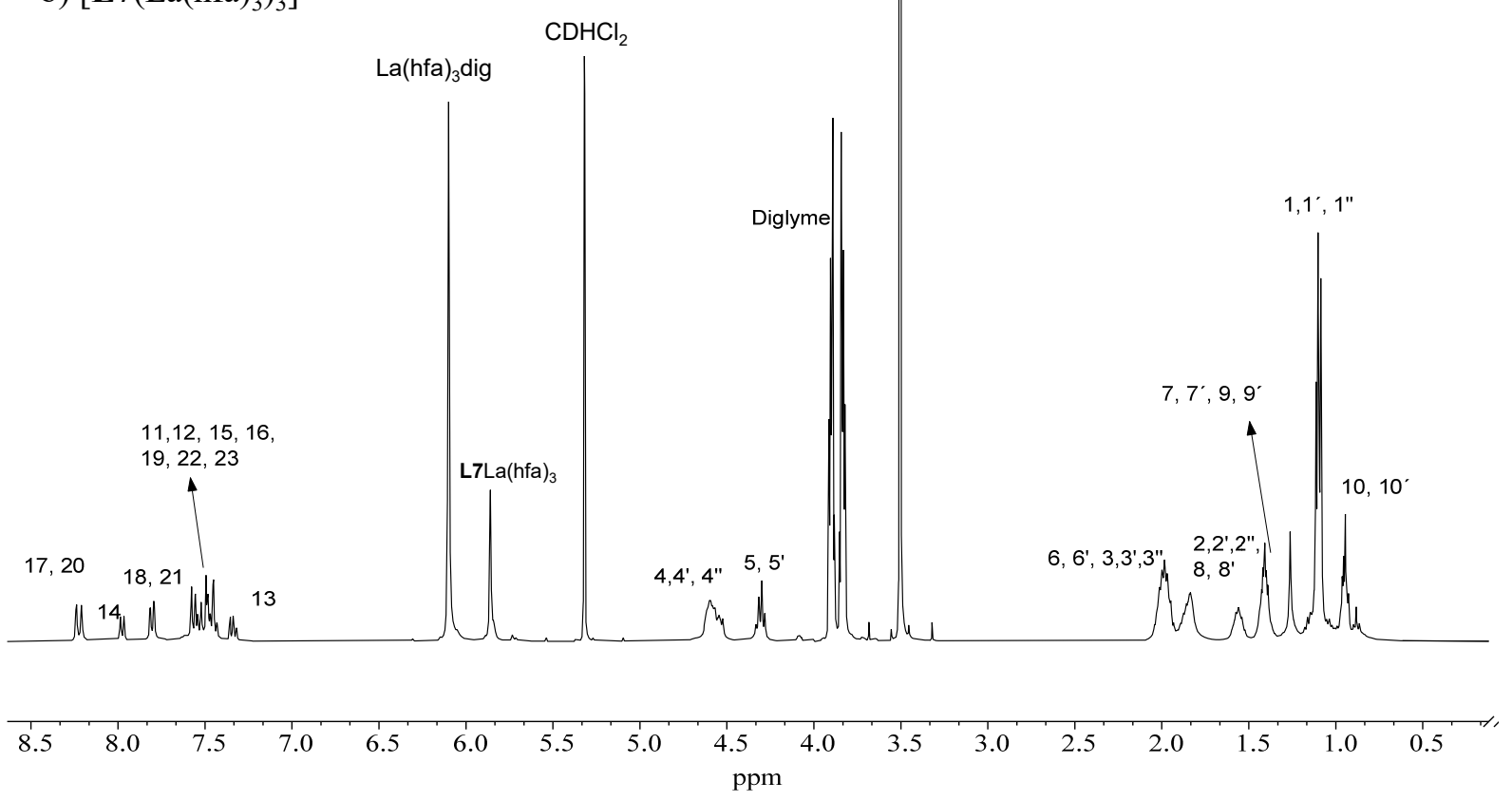

Figure S12. H NMR spectra with numbering scheme of a) $\left[\mathbf{L 7}\left(\mathrm{Eu}(\mathrm{hfa})_{3}\right)_{3}\right]$ and b) $\left[\mathbf{L 7}\left(\mathrm{La}(\mathrm{hfa})_{3}\right)_{3}\right]$ complexes in $\mathrm{CD}_{2} \mathrm{Cl}_{2}$ at $298 \mathrm{~K}$. 

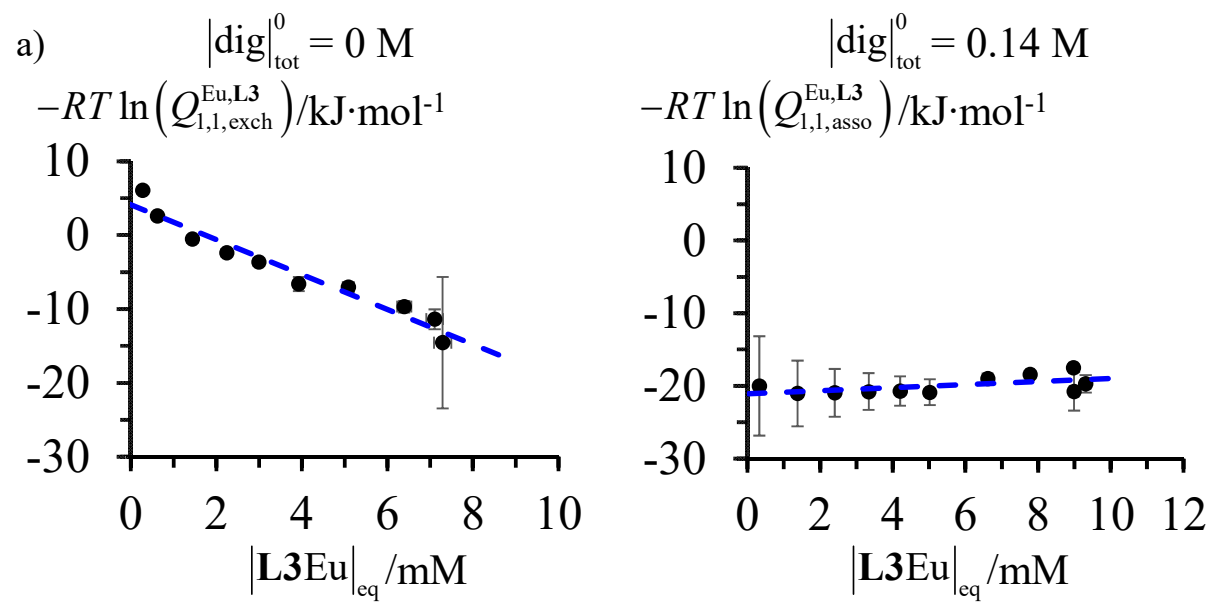

b)
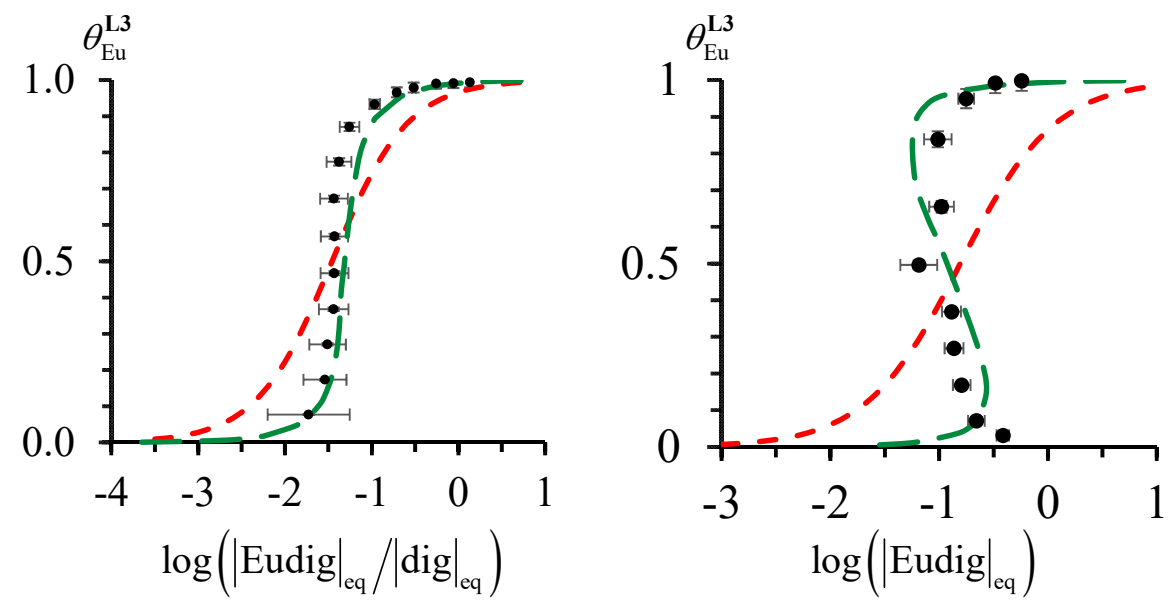

Figure S13. a) Dependences of the equilibrium reaction quotients $Q_{1,1, \mathrm{exch}}^{\mathrm{Eu} \mathbf{L} 3}\left(|\operatorname{dig}|_{\text {tot }}^{0}=0 \mathrm{M}\right.$, left part) and $Q_{1,1, \text { asso }}^{\mathrm{Eu}, \mathbf{L}}\left(|\operatorname{dig}|_{\text {tot }}^{0}=0.14 \mathrm{M}\right.$, right part) on the progress of the titration of $\mathbf{L 3}$ with [Eu(hfa) 3 dig] (eqn 8 ) and b) associated binding isotherms (black dots). The green traces correspond to the fits obtained with eqn (11) and variable activity coefficients, whereas the red traces are fitted with eqn (14) and constant activity coefficients. 


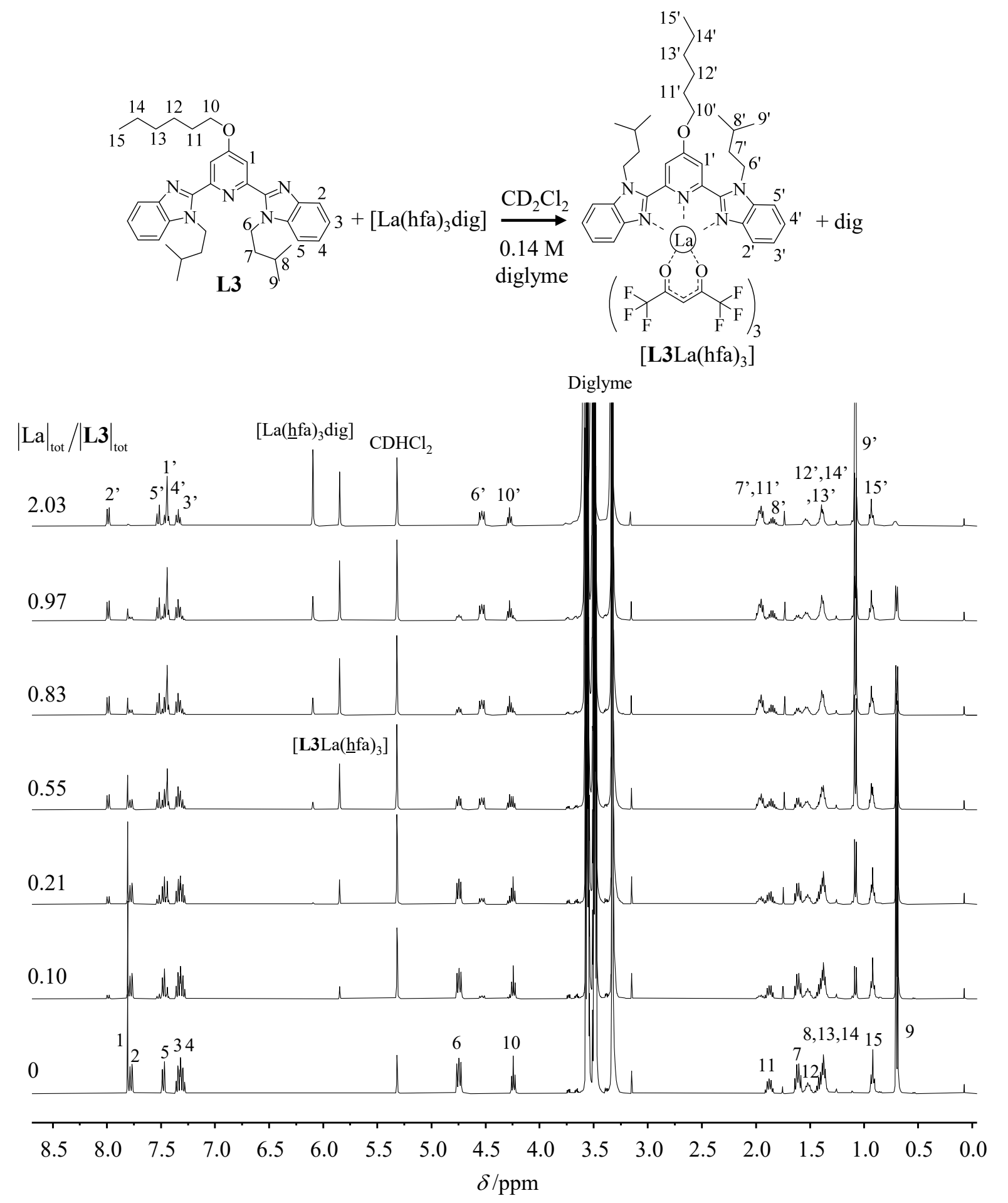

Figure S14. ${ }^{1} \mathrm{H}$ NMR titration of $\mathbf{L 3}(10 \mathrm{mM})$ with $\left[\mathrm{La}(\mathrm{hfa})_{3} \mathrm{dig}\right]$ in $\mathrm{CD}_{2} \mathrm{Cl}_{2}$ at $293 \mathrm{~K}\left(|\mathrm{dig}|_{\text {tot }}^{0}=0.14\right.$ M). 

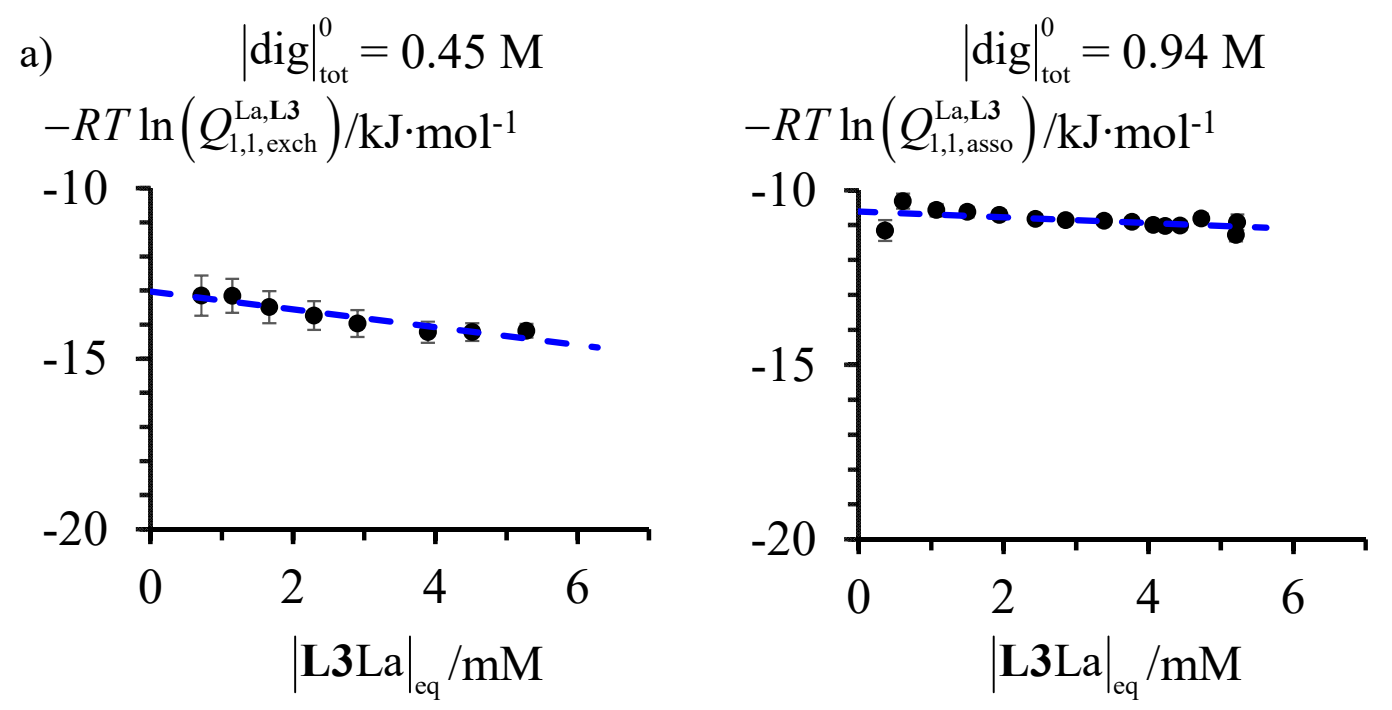

b)
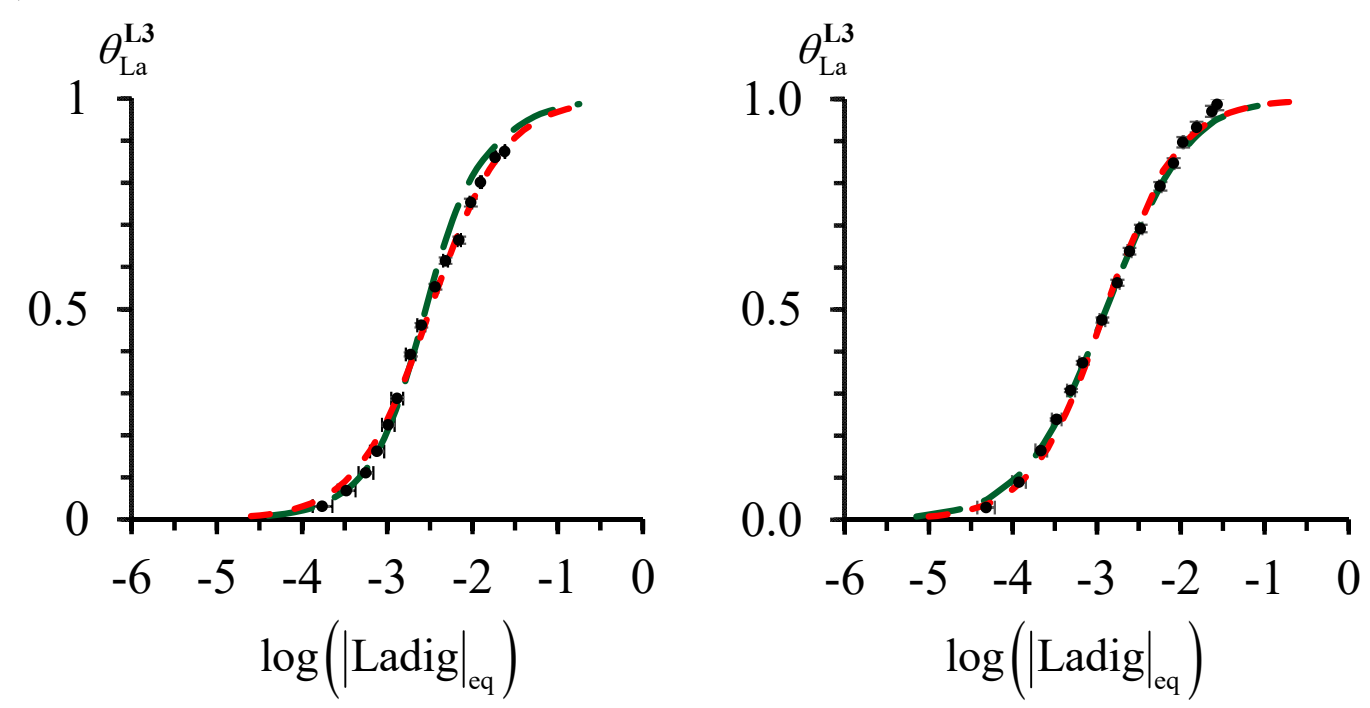

Figure S15. a) Dependences of the equilibrium reaction quotients $Q_{1,1, \mathrm{asso}}^{\mathrm{La} \mathbf{3} 3}\left(|\mathrm{dig}|_{\text {tot }}^{0}=0.45 \mathrm{M}\right.$, left part $|\operatorname{dig}|_{\text {tot }}^{0}=0.94 \mathrm{M}$, right part) on the progress of the titration of $\mathbf{L 3}$ with [La(hfa) 3 dig] (eqn 8) and b) associated binding isotherms (black dots). The green traces correspond to the fits obtained with eqn (11) and variable activity coefficients, whereas the red traces are fitted with eqn (14) and constant activity coefficients. 

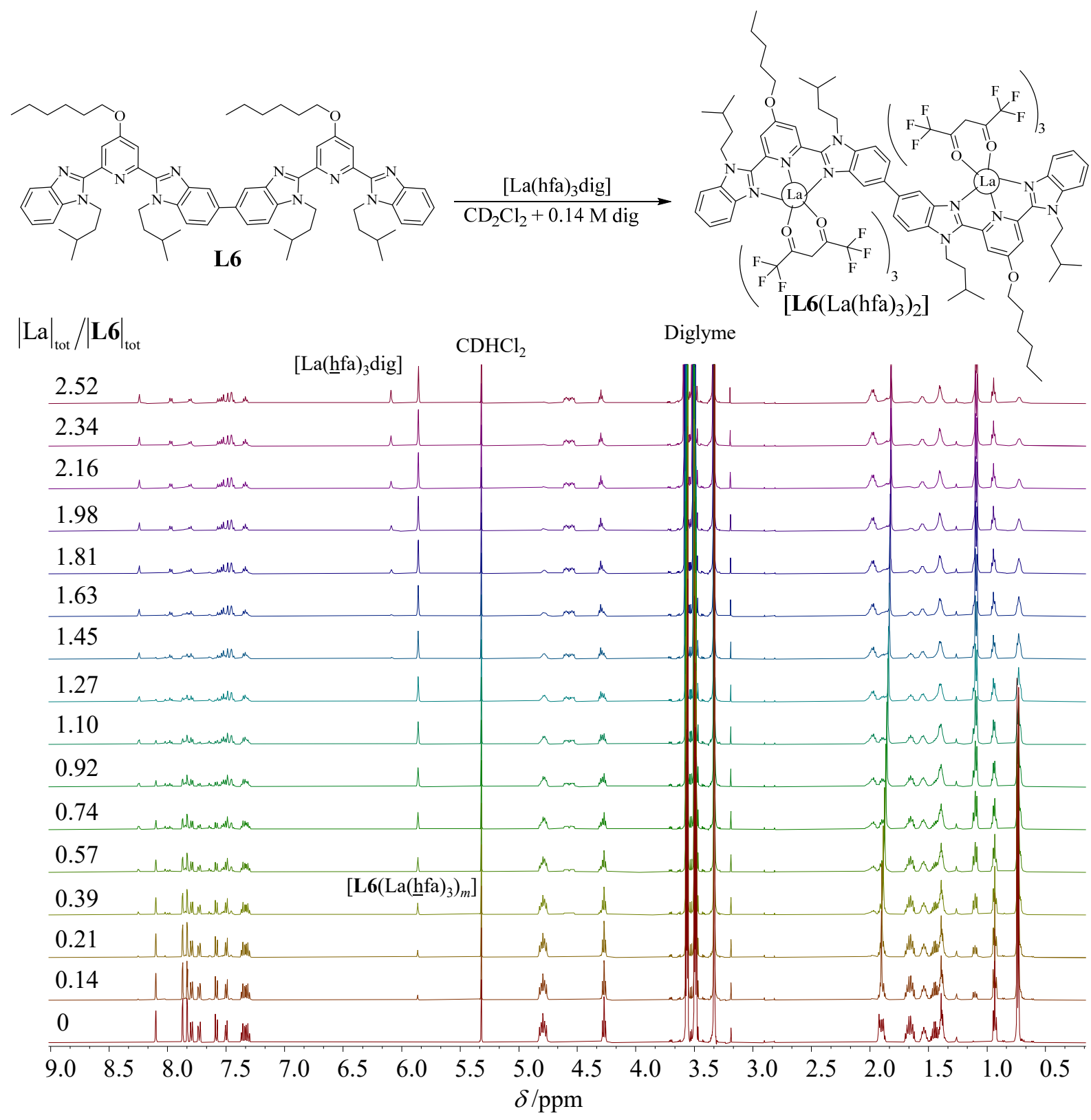

Figure S16. ${ }^{1} \mathrm{H}$ NMR titration of $\mathbf{L 6}(10 \mathrm{mM})$ with $\left[\mathrm{La}(\mathrm{hfa})_{3} \mathrm{dig}\right]$ in $\mathrm{CD}_{2} \mathrm{Cl}_{2}$ at $293 \mathrm{~K}\left(|\mathrm{dig}|_{\mathrm{tot}}^{0}=0.14\right.$ M). 

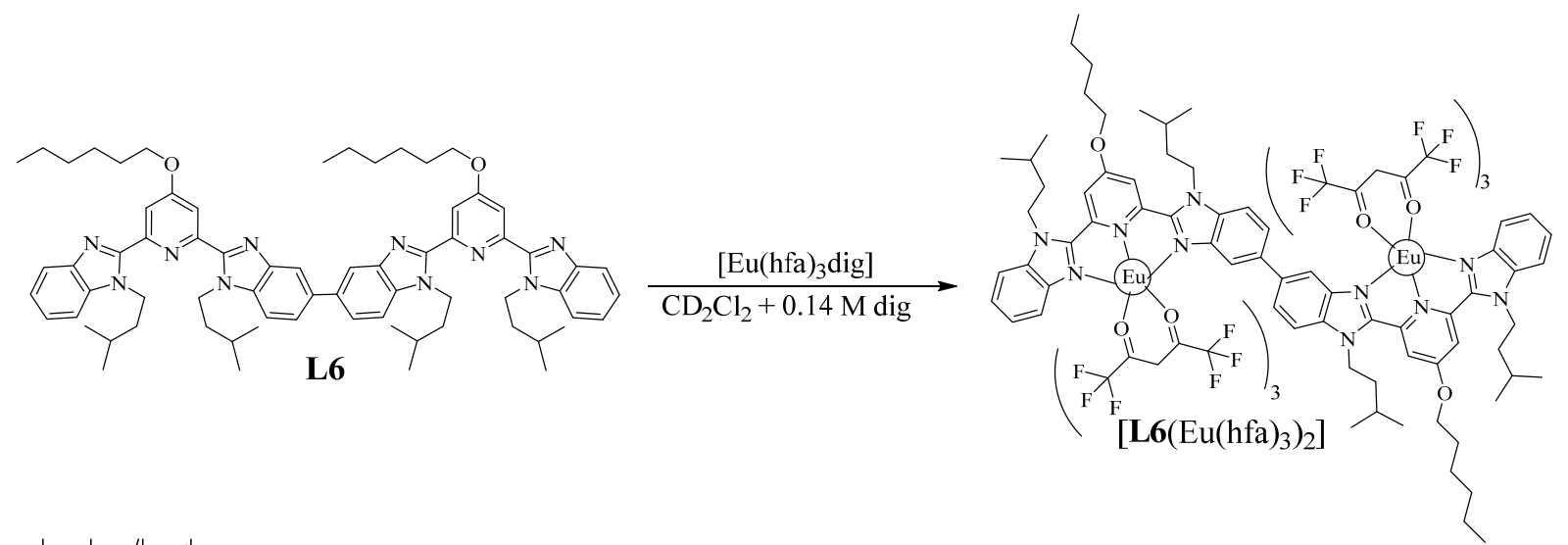

$|\mathrm{Eu}|_{\mathrm{tot}} /\left.\mathbf{L} \mathbf{6}\right|_{\mathrm{tot}}$

$\left[\mathbf{L 6}\left(\mathrm{Eu}(\mathrm{hfa})_{3}\right)_{2}\right]$

$\left[\mathrm{Eu}(\mathrm{hfa})_{3} \mathrm{dig}\right]$

\subsection{9}

2.45

2.24

2.02

1.81

1.59

1.38

1.17

0.96

0.75

0.54

[L6Eu(hfa $\left.)_{3}\right]$

0.33

0.12

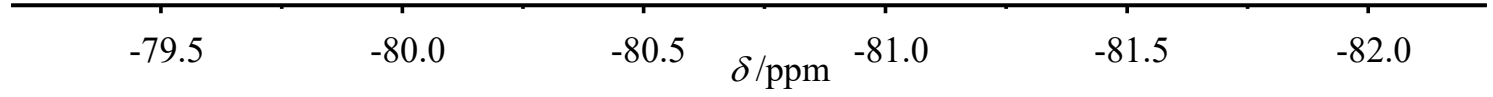

Figure S17. ${ }^{19} \mathrm{~F}$ NMR titration of $\mathbf{L 6}(10 \mathrm{mM})$ with [Eu(hfa $\left.)_{3} \mathrm{dig}\right]$ in $\mathrm{CD}_{2} \mathrm{Cl}_{2}$ at $293 \mathrm{~K}\left(|\mathrm{dig}|_{\text {tot }}^{0}=0.14\right.$ M). 


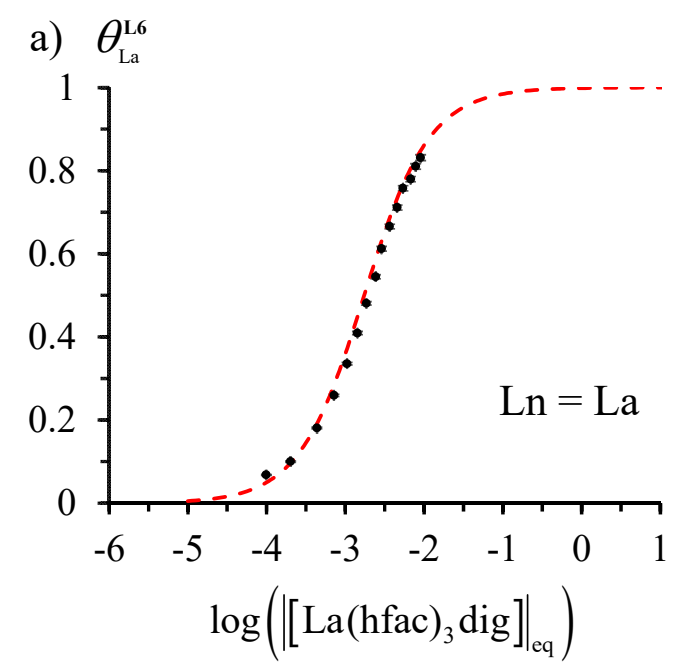

b) $\theta_{\mathrm{Eu}}^{\text {L6 }}$
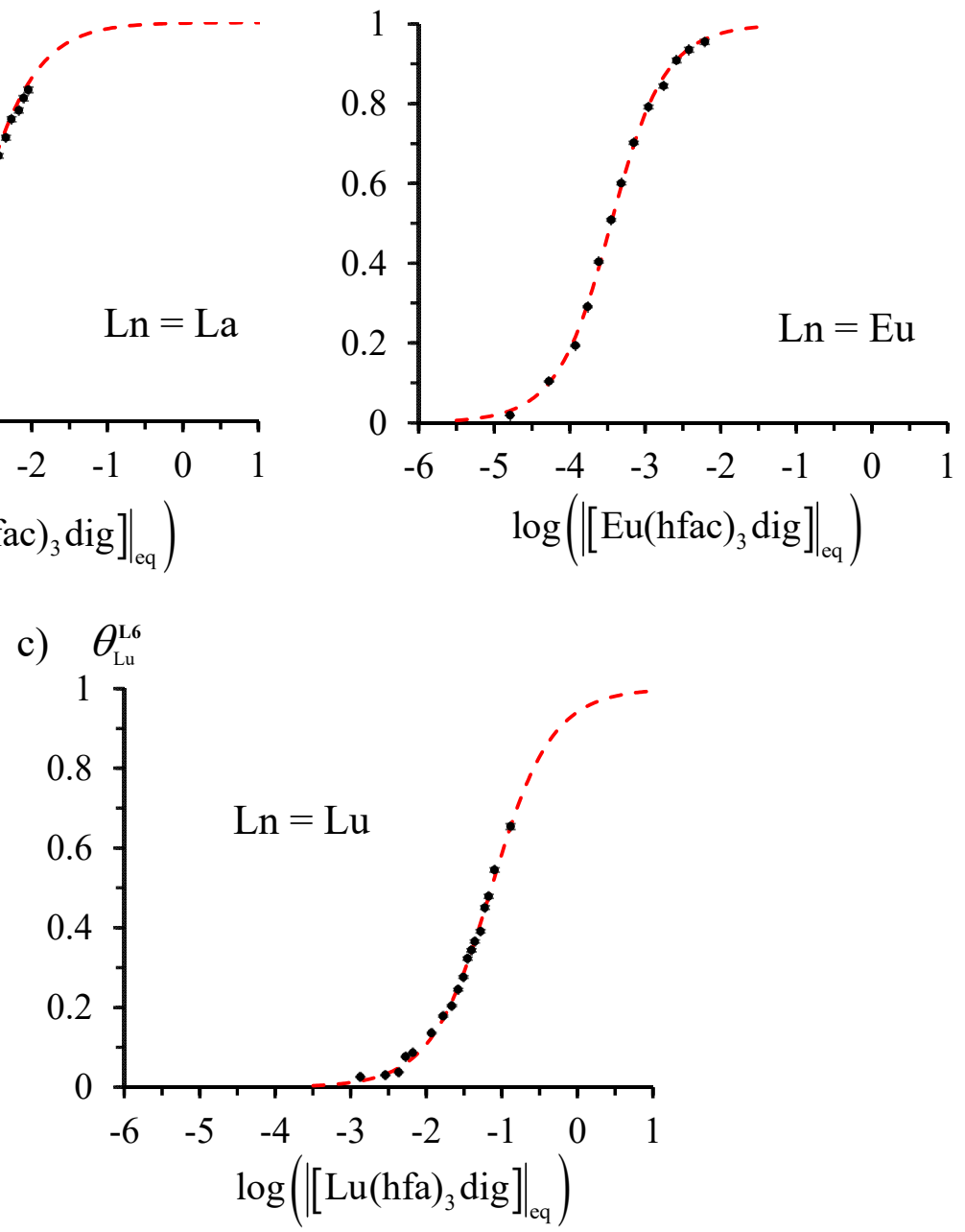

Figure S18. Binding isotherms (black dots) for the titration of $\mathbf{L 6}$ with [ $\left.\mathrm{Ln}(\mathrm{hfa})_{3} \mathrm{dig}\right]$ in $\mathrm{CD}_{2} \mathrm{Cl}_{2}+$ $|\operatorname{dig}|_{\text {tot }}^{0}=0.14 \mathrm{M}$. The red traces are fitted with eqn (15) and stability constants collected in Table 4 . 

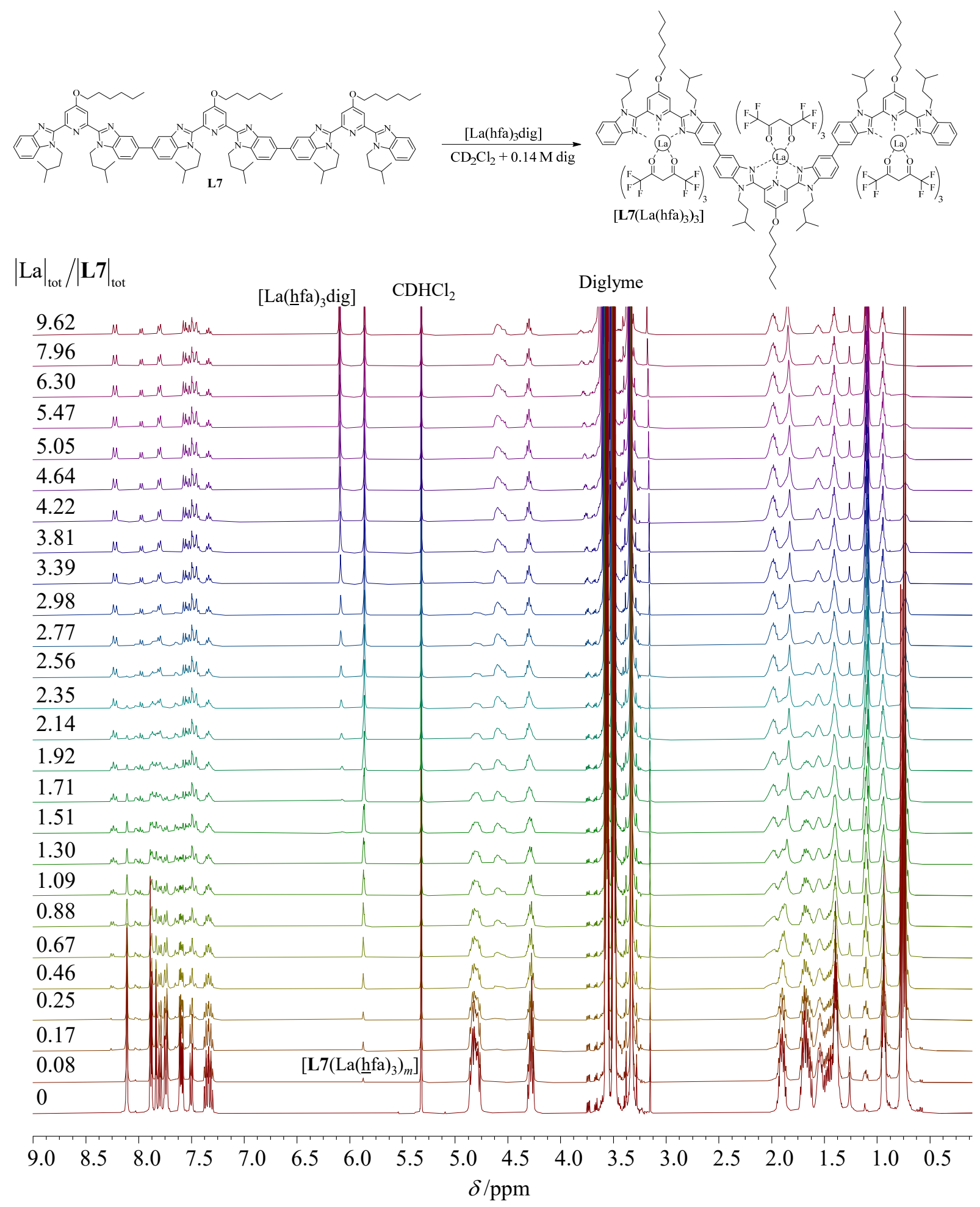

Figure S19. ${ }^{1} \mathrm{H}$ NMR titration of $\mathbf{L 7}(10 \mathrm{mM})$ with $\left[\mathrm{La}(\mathrm{hfa})_{3} \mathrm{dig}\right]$ in $\mathrm{CD}_{2} \mathrm{Cl}_{2}$ at $293 \mathrm{~K}\left(|\mathrm{dig}|_{\mathrm{tot}}^{0}=0.14\right.$ M). 

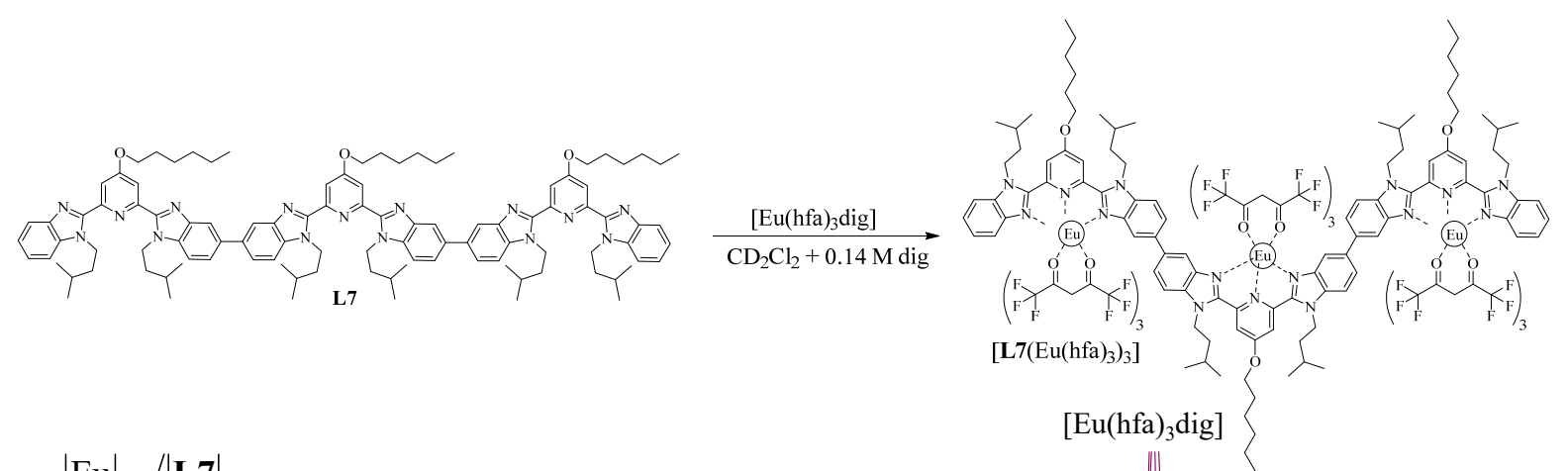

$|\mathrm{Eu}|_{\text {tot }} /|\mathbf{L} 7|_{\text {tot }}$

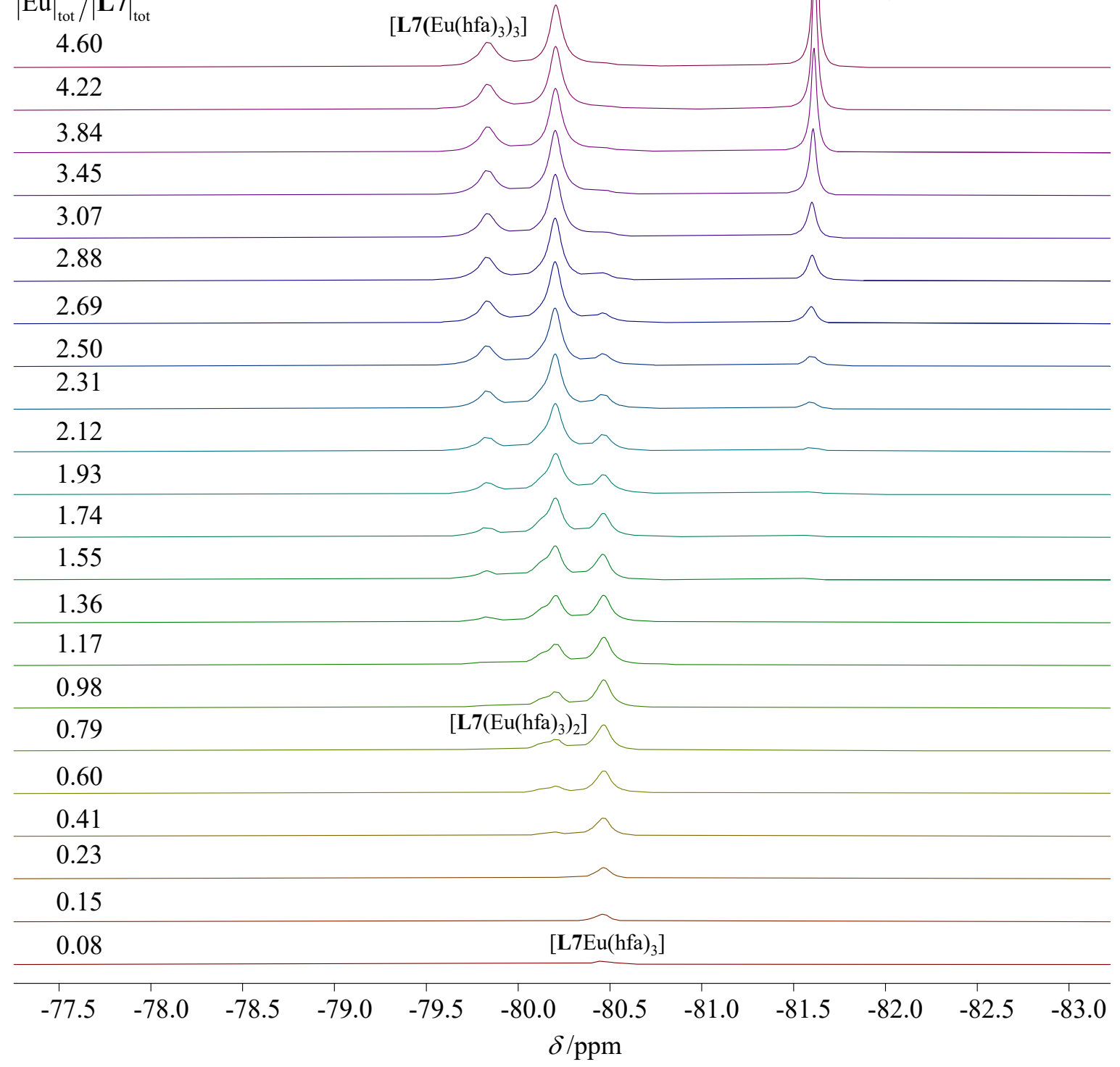

Figure S20. ${ }^{19} \mathrm{~F}$ NMR titration of $\mathbf{L} 7(10 \mathrm{mM})$ with $\left[\mathrm{Eu}(\mathrm{hfa})_{3} \operatorname{dig}\right]$ in $\mathrm{CD}_{2} \mathrm{Cl}_{2}$ at $293 \mathrm{~K}\left(|\mathrm{dig}|_{\text {tot }}^{0}=0.14\right.$ M). 


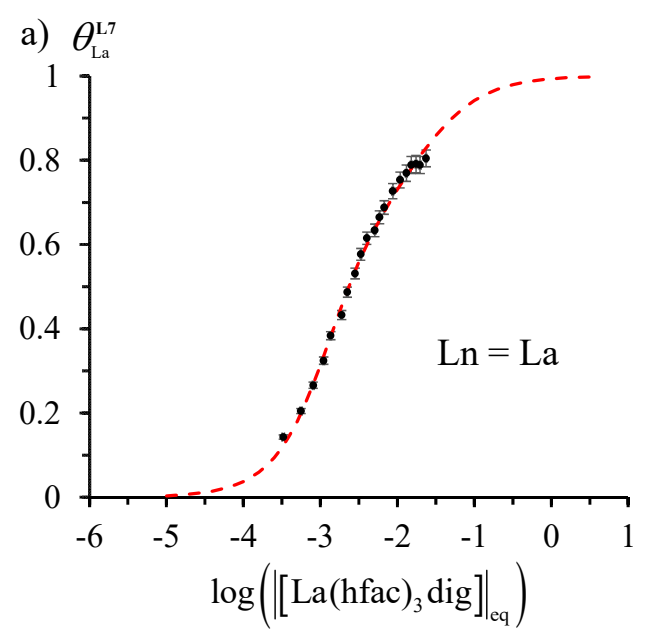

b) $\theta_{\mathrm{Eu}}^{\mathrm{L} 7}$
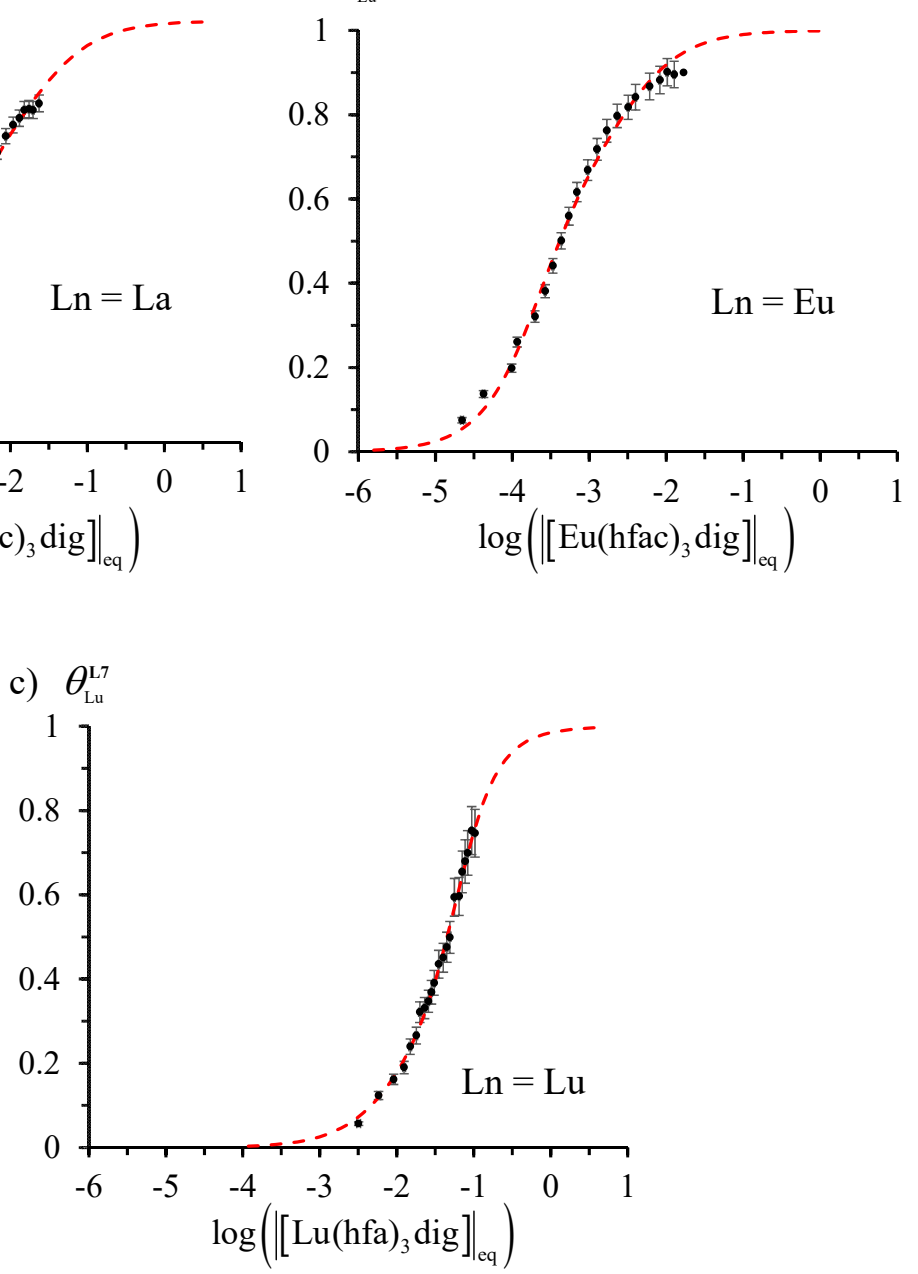

Figure S21. Binding isotherms (black dots) for the titration of $\mathbf{L 7}$ with [ $\left.\mathrm{Ln}(\mathrm{hfa})_{3} \mathrm{dig}\right]$ in $\mathrm{CD}_{2} \mathrm{Cl}_{2}+$ $|\operatorname{dig}|_{\text {tot }}^{0}=0.14 \mathrm{M}$. The red traces are fitted with eqn (15) and stability constants collected in Table 4 . 

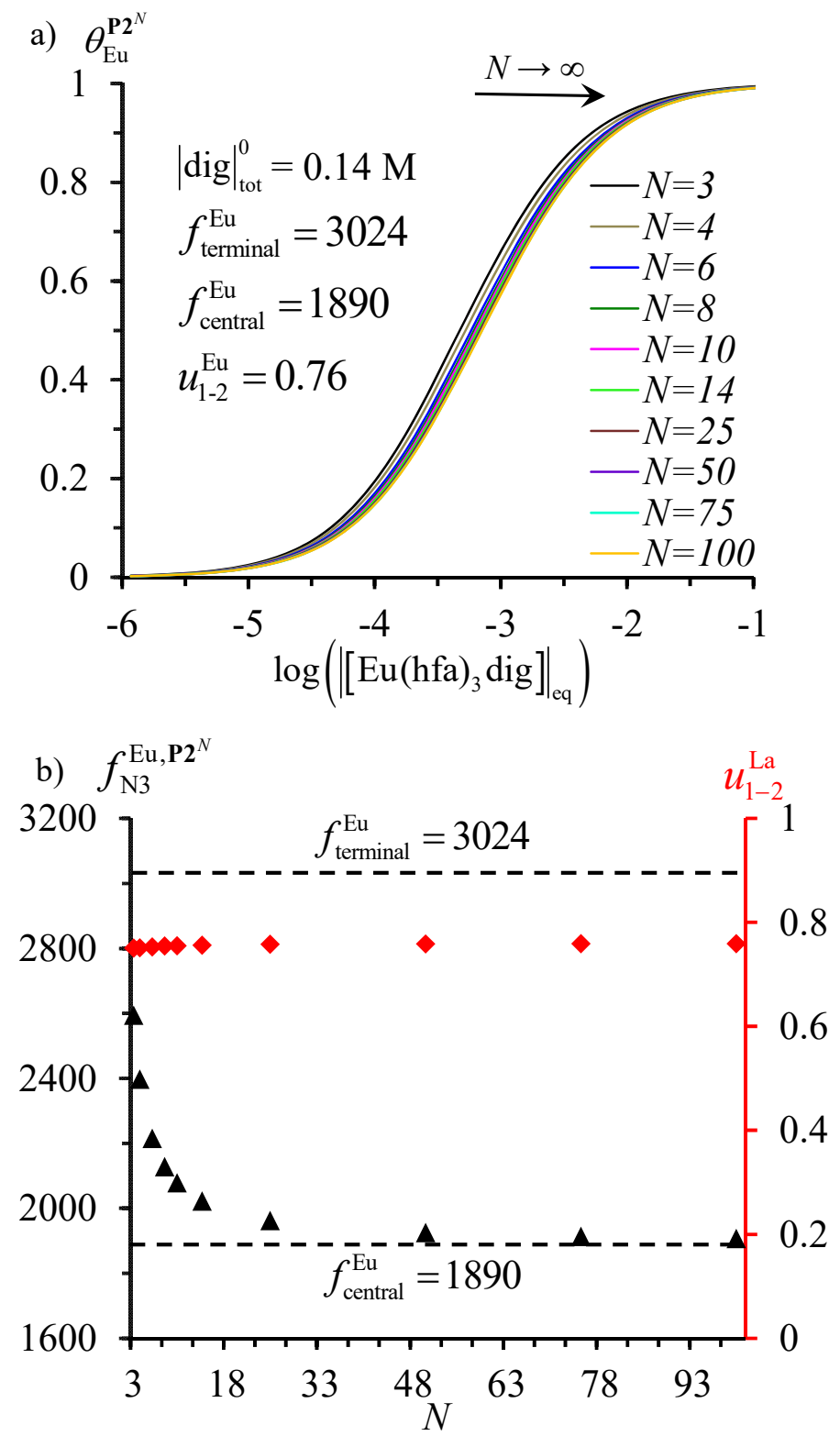

Figure S22. a) Binding isotherms predicted for $\left[\mathbf{P 2}^{N}\left(\mathrm{Eu}(\mathrm{hfa})_{3}\right)_{m}\right]$ in $\mathrm{CD}_{2} \mathrm{Cl}_{2}\left(|\operatorname{dig}|_{\text {tot }}^{0}=0.14 \mathrm{M}\right)$ computed with eqns (22)-(24) and b) average intrinsic affinities $f_{\mathrm{N} 3}^{\mathrm{Eu}, \mathbf{P}^{N}}$ (black triangles) and intersite interactions $u_{1-2}^{\mathrm{Eu}}$ (red diamonds) obtained with eqn (25) which considers that all the binding sites have the same affinity along the linear oligomers of length $N$. 

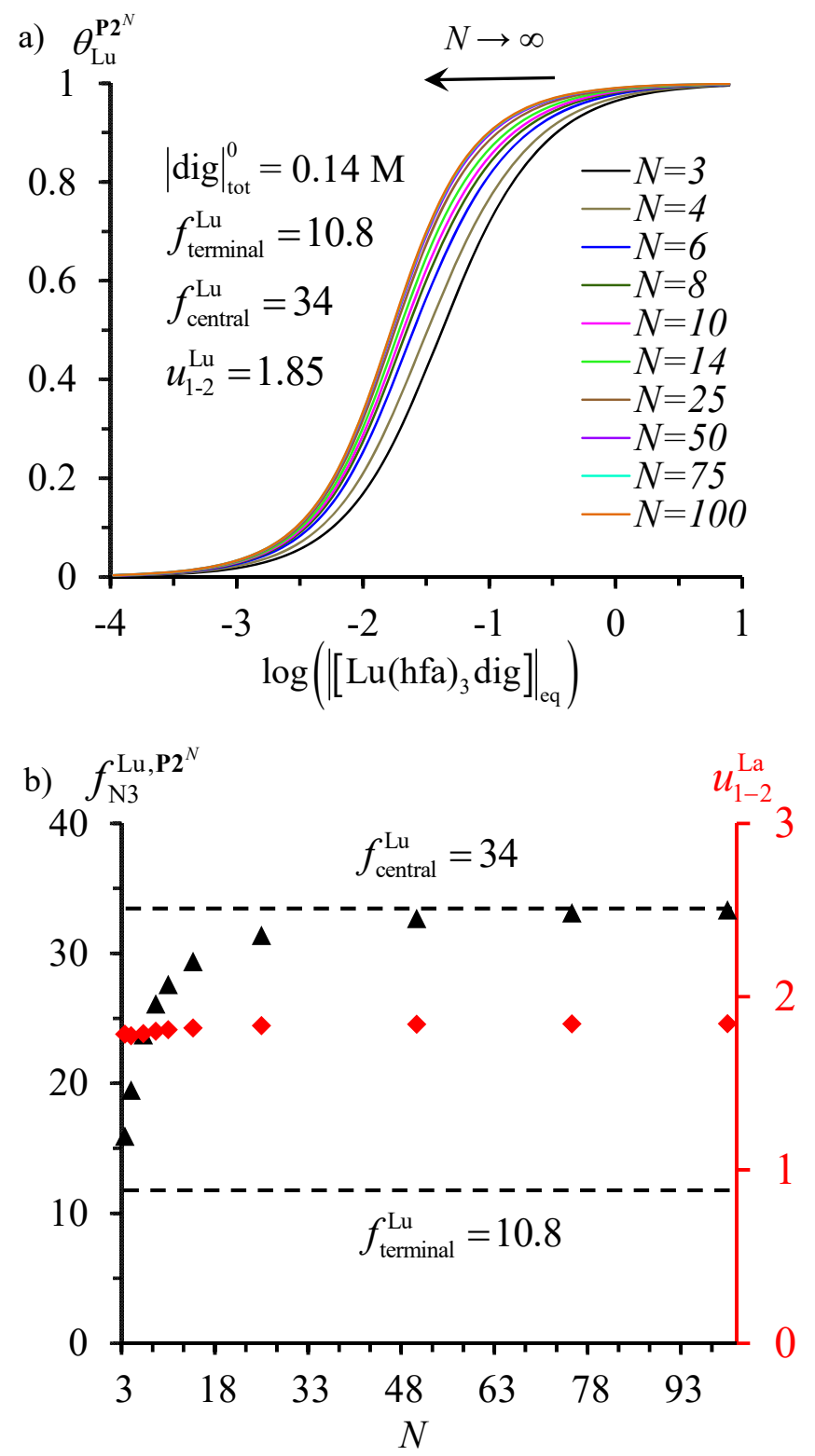

Figure S23. a) Binding isotherms predicted for $\left[\mathbf{P 2}^{N}\left(\mathrm{Lu}(\mathrm{hfa})_{3}\right)_{m}\right]$ in $\mathrm{CD}_{2} \mathrm{Cl}_{2}\left(|\operatorname{dig}|_{\text {tot }}^{0}=0.14 \mathrm{M}\right)$ computed with eqns (22)-(24) and b) average intrinsic affinities $f_{\mathrm{N} 3}^{\mathrm{Lu}, \mathbf{P}^{N}}$ (black triangles) and intersite interactions $u_{1-2}^{\mathrm{Lu}}$ (red diamonds) obtained with eqn (25) which considers that all the binding sites have the same affinity along the linear oligomers of length $N$. 